\title{
The Mobility Impact in IEEE 802.11p Infrastructureless Vehicular Networks
}

\author{
by \\ Waleed Alasmary
}

\author{
A thesis \\ presented to the University of Waterloo \\ in fulfillment of the \\ thesis requirement for the degree of \\ Master of Applied Science \\ in \\ Electrical and Computer Engineering
}

Waterloo, Ontario, Canada, 2010

(c) Waleed Alasmary 2010 
I hereby declare that I am the sole author of this thesis. This is a true copy of the thesis, including any required final revisions, as accepted by my examiners.

I understand that my thesis may be made electronically available to the public. 


\begin{abstract}
Vehicular ad hoc networks (VANETs) are an extreme case of mobile ad hoc networks (MANETs). High speed and frequent network topology changes are the main characteristics of vehicular networks. These characteristics lead to special issues and challenges in the network design, especially at the medium access control (MAC) layer. Due to high speed of nodes and their frequent disconnections, it is difficult to design a MAC scheme in VANETs that satisfies the quality-of-service requirements in all networking scenarios. In this thesis, we provide a comprehensive evaluation of the mobility impact on the IEEE 802.11p MAC performance. The study evaluates basic performance metrics such as packet delivery ratio, throughput, and delay, as well as the impact of mobility factors. The study also presents a relation between the mobility factors and the respective medium access behavior. Moreover, a new unfairness problem according to node relative speed is identified for both broadcast and unicast scenarios. To achieve better performance, we propose two dynamic contention window mechanisms to alleviate network performance degradation due to high mobility. Extensive simulation results show the significant impact of mobility on the IEEE 802.11p MAC performance, an identification of a new unfairness problem in the vehicle-to-vehicle $(\mathrm{V} 2 \mathrm{~V})$ communications, and the effectiveness of the proposed MAC schemes.
\end{abstract}




\section{Acknowledgements}

I would like to express my deepest appreciation and gratitude to my supervisor Professor Weihua Zhuang for her guidance, support, and share of knowledge. Professor Zhuang can be considered as a role model for the professional academic supervisor. She gave me the opportunity to choose the topic of my Master's research, and provided me with invaluable suggestions and advice. She always inspired me when discussing research problems. In addition to her solid knowledge that enriched my research, she was always available, professional, helpful, and extremely nice. Her extraordinary commitment to the excellence of research made the opportunity to work with her a true reward of my Master's research.

I would like to thank Professor Xuemin (Sherman) Shen and Professor Liang-Liang Xie for serving as members of my thesis committee. Their encouragement and comments are highly appreciated.

I would like to thank my colleagues at the broadband communication research (BBCR) group. The weekly group meeting broadened my knowledge in the area of wireless communication networks.

Special thanks to Umm Al-Qura University for giving me a scholarship to pursue my Master's degree. I would like to thank the Ministry of Higher Education at Saudi Arabia and the Saudi Arabian Cultural Bureau in Canada for their support.

I am truly indebted to my parents, my brother Hassan, and my sister May for their endless support, encouragement, and prayers.

I can not express my gratitude to my wife, Rabab, for her constant support. Without her encouragement, this thesis would not be completed. 
Finally, I would like to thank all my friends in University of Waterloo, who helped me through the course of my studies, discussed ideas about my research, and made my life at Waterloo enjoyable.

Thank you all ! 


\section{Dedication}

To my parents

To my lovely wife, Rabab

To my precious daughter, Leen 


\section{Contents}

List of Tables $\quad$ xi

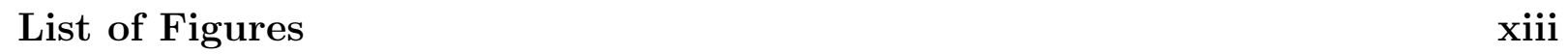

$\begin{array}{ll}\text { List of Abbreviations } & \text { xiv }\end{array}$

\begin{tabular}{lll}
\hline & Introduction & 1
\end{tabular}

1.1 Vehicular communication networks . . . . . . . . . . . . . . 1

1.2 Vehicular networks applications . . . . . . . . . . . . . . . 2

1.3 Motivation and Research Contributions . . . . . . . . . . . . . . . . . 3

1.4 Thesis Organization $\ldots \ldots \ldots \ldots$. . . . . . . . . . . . . . . . 6

\begin{tabular}{lll}
\hline 2 & Literature Review and Related Work & 7
\end{tabular}

2.1 MAC Protocols for Vehicular Ad Hoc Networks . . . . . . . . . . . . . . . 7

2.2 Quality-of-Service Metrics . . . . . . . . . . . . . . . . . . . 9

$2.3 \quad$ Challenges and Issues in VANET Environment . . . . . . . . . . . . . . . . 11 
$2.3 .1 \quad$ High Speed and Frequent Topology Changes . . . . . . . . . . . . 11

2.3 .2 Lack of central coordination . . . . . . . . . . . . . . . . 12

$2.3 .3 \quad$ Scalability . . . . . . . . . . . . . . . . . . . . . . . 12

$2.3 .4 \quad$ Hidden and Exposed Node Problems $\ldots \ldots \ldots$

$2.4 \quad$ IEEE 802.11 based protocols $\ldots \ldots \ldots \ldots \ldots$

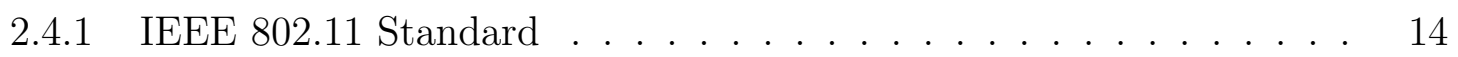

$2.4 .2 \quad$ IEEE $802.11 \mathrm{p}$, WAVE and DSRC $\ldots \ldots \ldots \ldots \ldots$

2.4 .3 Related Work Review . . . . . . . . . . . . . . . . . . . 18

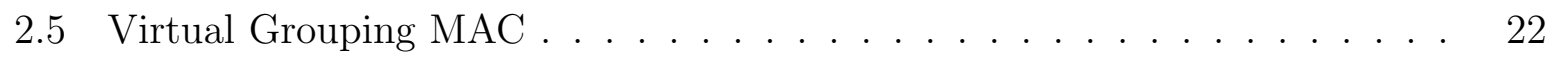

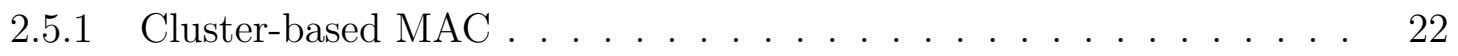

$2.5 .2 \quad$ Space Division Multiple Access (SDMA) $\ldots \ldots \ldots \ldots \ldots \ldots$

$2.5 .3 \quad$ Token-Ring based MAC $\ldots \ldots \ldots \ldots \ldots \ldots$

2.6 Other protocols $\ldots \ldots \ldots \ldots \ldots$

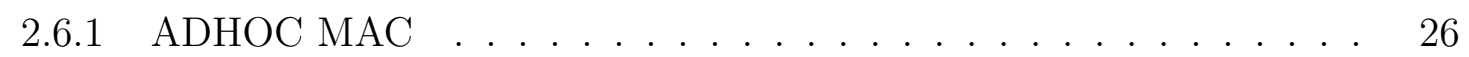

2.6 .2 Directional Antenna-Based MAC $\ldots \ldots \ldots \ldots \ldots$

$2.6 .3 \quad$ Repetition-based MAC $\ldots \ldots \ldots \ldots \ldots \ldots$

2.6 .4 Multichannel MAC . . . . . . . . . . . . . . . . . . . 30

2.7 Comparison of the Existing Protocols $\ldots \ldots \ldots$

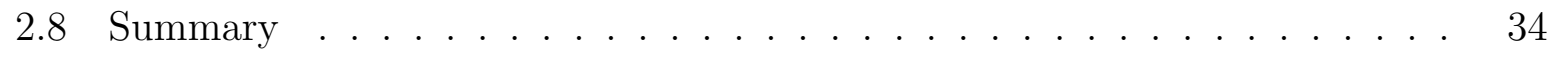


3 System Model and Mobility Adaptive Schemes 35

3.1 Network Topology and Configuration . . . . . . . . . . . . . . . . . . 37

$3.2 \quad$ MAC Layer $\ldots \ldots \ldots \ldots$

$3.3 \quad$ MAC Adaptivity to Mobility . . . . . . . . . . . . . . . . . . . . . . . . . . . . . 39

$3.3 .1 \quad$ Adaptation to the Number of Neighboring Nodes $\ldots \ldots \ldots$. . . . 40

3.3 .2 Adaptation to Vehicle Velocity $\ldots \ldots \ldots \ldots$

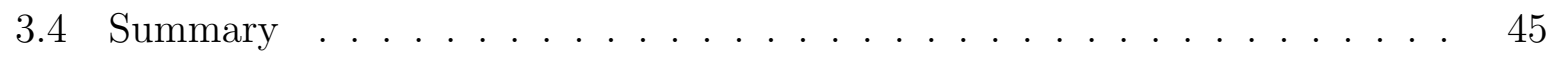

\begin{tabular}{llr}
\hline & Performance Evaluation & 46
\end{tabular}

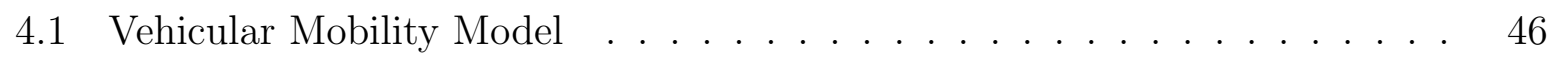

4.2 Simulation Parameters $\ldots \ldots \ldots \ldots$

$4.3 \quad$ Mobility and Performance Metrics . . . . . . . . . . . . . . . . . . . . 49

$4.4 \quad$ Evaluation of the Mobility Impact $\ldots \ldots \ldots \ldots$

4.4 .1 Broadcast Scenario . . . . . . . . . . . . . . . . . . . . . . . . 51

4.4 .2 Unicast Scenario . . . . . . . . . . . . . . . . . . . . 55

$4.4 .3 \quad$ Evaluation of the IEEE $802.11 \mathrm{p}$ Performance $\ldots \ldots \ldots \ldots \ldots$

4.5 Evaluation of the Adaptive Schemes . . . . . . . . . . . . . . . . 64

4.6 Summary $\ldots \ldots \ldots \ldots \ldots \ldots$

$\begin{array}{lll}5 \text { Conclusions and Future Work } & 73\end{array}$

5.1 Research Contributions . . . . . . . . . . . . . . . . . . . . . . 73

5.2 Future Work . . . . . . . . . . . . . . . . . . . . . . . . . . . . . 75

ix 
\begin{tabular}{|rr}
\hline Bibliography & 84
\end{tabular} 


\section{List of Tables}

1.1 Main characteristics of mobile and vehicular ad hoc networks. . . . . . . . 2

$2.1 \quad$ Comparison between the current VANET MAC protocols. . . . . . . . . . 34

3.1 Different parameters values for different traffic categories in 802.11p EDCA. 38

3.2 List of notations and variables used in the model. . . . . . . . . . . . . . . 44

$3.3 \quad$ Dynamic service priority assignment based on relative speed. . . . . . . . . 45

$4.1 \quad$ Different parameters for the simulation. . . . . . . . . . . . . . . . . . . . . 49

$4.2 \quad$ Dynamic assignment of parameters according to relative speed. . . . . . . . 64 


\section{List of Figures}

1.1 An illustration of V2V and V2I communication modes in VANETs. . . . . 3

1.2 The problem of unfairness in channel access due to nodes having different speeds. . . . . . . . . . . . . . . . . . . . 5

2.1 Hidden and exposed node problems. . . . . . . . . . . . . . . . . . . 13

2.2 Solving hidden node problem using the RTS/CTS handshaking. . . . . . . 14

$2.3 \quad$ DSRC spectrum allocation by FCC. . . . . . . . . . . . . . . . . . . . 16

2.4 The IEEE 1609 standards. . . . . . . . . . . . . . . . . . . . . . . . 18

$2.5 \quad$ Clustering structure example. . . . . . . . . . . . . . . . . . . . . . . 23

2.6 Example of inter-cluster interference. . . . . . . . . . . . . . . . . . . . . . 24

$2.7 \quad$ Illustration of the idea of repetition for two senders A and B. . . . . . . . . 29

$3.1 \quad$ Illustration of the DCF operation. . . . . . . . . . . . . . . . . . 38

$3.2 \quad$ Frame structure with periodic broadcasting of vehicle information. . . . . . 39

3.3 Illustration of a virtual transmission time. . . . . . . . . . . . . . . . . . . . 42

$4.1 \quad$ Vehicles mobility according to the freeway model. . . . . . . . . . . . . . . 47 
4.2 The traffic mobility scenario used in the simulation. . . . . . . . . . . . . . 48

$4.3 \quad$ Factors affecting the IEEE 802.11 p performance. . . . . . . . . . . . . . . . 50

$4.4 \quad$ Probability mass function of the number of neighbors. . . . . . . . . . . . . 52

4.5 Average number of communication neighbors. . . . . . . . . . . . . . 52

4.6 CDF of the communication duration of a link. . . . . . . . . . . . . 53

4.7 Accumulated fraction of time access versus relative speed. . . . . . . . . . 54

4.8 Accumulated fraction of time access versus distance. . . . . . . . . . . . . . 55

4.9 Mobility impact at the MAC layer in the unicast scenario. . . . . . . . . . 56

4.10 Accumulated fraction of access time versus distance. . . . . . . . . . . . . . 57

4.11 PDR of the IEEE 802.11p. . . . . . . . . . . . . . . . . . . . 59

4.12 Average number of retransmissions of the IEEE 802.11p. . . . . . . . . . . 60

4.13 Throughput of the IEEE 802.11p. . . . . . . . . . . . . . . . . . . . . . 61

4.14 Transmission delay of the IEEE 802.11 p. . . . . . . . . . . . . . . . . . . 62

4.15 Jain Fairness Index of the IEEE 802.11p. . . . . . . . . . . . . . . . . . . 63

4.16 PDR of the adaptive MAC schemes. . . . . . . . . . . . . . . . . . . 66

4.17 Average number of retransmissions of the adaptive MAC schemes. . . . . . 67

4.18 Throughput of the adaptive MAC schemes. . . . . . . . . . . . . . . . . . 68

4.19 Transmission delay of the adaptive MAC schemes. . . . . . . . . . . . . . . 69

4.20 Jain Fairness Index of the adaptive MAC schemes. . . . . . . . . . . . . . . 70 


\section{List of Abbreviations}

\begin{tabular}{ll} 
AC/TC & Access/traffic category \\
AOA & Angle of arrival \\
BP & Access point \\
BSSID & Basic service set \\
CBR & Basic service set identification \\
CBT & Constant bit rate \\
CCDF & Channel busy time \\
CCH & Complementary commutative distribution function \\
CDMA & Control channel \\
CH & Code division multiple access \\
CMV & Clusterhead \\
CSMA & Cognitive VANET MAC \\
CSMA/CA & Carrier sense multiple access \\
CSMA/CD & CSMA with collision avoidance \\
CTS & CSMA with collision detection \\
CW & Clear-to-send \\
\hline
\end{tabular}




\begin{tabular}{ll} 
DCA & Dynamic channel assignment \\
DCAP & Dedicated coordinating access point \\
DSDV & Destination-sequenced distance-vector \\
DCF & Distributed coordination function \\
DSRC & Dedicated short range communication \\
EDCA & Enhanced distributed channel access \\
FCC & Federal communication commission \\
FDMA & Frequency division multiple access \\
GPS & Global positioning system \\
IBSS & Independent basic service set \\
ITS & Intelligent transportation system \\
MAC & Medium access control \\
MANET & Mobile ad hoc networks \\
NS2 & Network simulator \\
OCC & Optical orthogonal codes \\
OTRP & overlay token ring protocol \\
PCF & Point coordination function \\
PDR & Packet delivery ratio \\
PLR & Packet loss ratio \\
QoS & Quality-of-service \\
R-ALOHA & Reservation ALOHA \\
RR-ALOHA & Reliable R-ALOHA \\
RSU & Roadside unit \\
RTS & Request-to-send \\
\hline
\end{tabular}




$\begin{array}{ll}\text { S-ALOHA } & \text { Slotted ALOHA } \\ \text { SDMA } & \text { Space division multiple access } \\ \text { SNR } & \text { Signal to noise ratio } \\ \text { TDMA } & \text { Time division multiple access } \\ \text { VANET } & \text { Vehicular ad hoc networks } \\ \text { V2I } & \text { Vehicle-to-infrastructure } \\ \text { V2V } & \text { Vehicle-to-vehicle } \\ \text { VO } & \text { Voice } \\ \text { VI } & \text { Video } \\ \text { WAVE } & \text { Wireless access in vehicular networks } \\ \text { WBSS } & \text { WAVE basic service set } \\ \text { WLAN } & \text { Wireless LAN } \\ \text { WTRP } & \text { Wireless token ring protocol } \\ \text { W-UIM } & \text { WBSS user initiation mode }\end{array}$




\section{Chapter 1}

\section{Introduction}

\subsection{Vehicular communication networks}

Vehicular ad hoc networks (VANETs) are an extreme case of mobile ad hoc networks (MANETs). In MANETs, nodes communicate with each other in an ad hoc mode, i.e., without a fixed infrastructure. In VANETs, the nodes communicate in a similar way, but with high speed and different mobility characteristics which lead to frequent network topology changes. On the other hand, several characteristics of the vehicular networks can be used as advantages [1]. For example, in VANETs, vehicles move within specific road directions, and the importance of transmission power and message storage space constraints is not limited. Moreover, the geographic location of a vehicle can be determined by using a global positioning system (GPS) receiver, when applicable. Table 1.1 lists the main characteristics of mobile and vehicular ad hoc networks. 


\begin{tabular}{lll}
\hline & MANETs & VANETs \\
\hline Topology & dynamic & highly dynamic \\
Speed & low & high \\
Mobility & unconstrained & constrained \\
Power \& buffer space & very limited & not much limited \\
Infrastructure & not fixed & fixed/not fixed \\
\hline
\end{tabular}

Table 1.1: Main characteristics of mobile and vehicular ad hoc networks.

\subsection{Vehicular networks applications}

The main purpose of communication in vehicular networks, either in vehicle-to-vehicle $(\mathrm{V} 2 \mathrm{~V})$ or vehicle-to-infrastructure (V2I), is to provide safety and/or non-safety services. In safety applications, vehicles broadcast safety messages to other vehicles within a small range such as 300 meters [2]. Safety messages are given the highest priority in vehicular communication networks. However, even with the consideration of priority, when a vehicle broadcasts a safety message, a transmission collision may occur due to a transmission of other safety messages that needs to be sent with priority by simultaneously. Thus, an efficient medium access methodology is needed to enhance the collision avoidance process. Another application for vehicular networks is multimedia services. For example, a vehicle might download maps, audio, or video files with certain quality-of-service (QoS) requirement from a roadside unit (RSU) or from another vehicle in a cooperative mode. If these applications are provided commercially, ceratin QoS should be guaranteed. This makes designing an efficient medium access control (MAC) protocol in vehicular networks essential. Both V2V and V2I communication modes are illustrated in Figure 1.1 


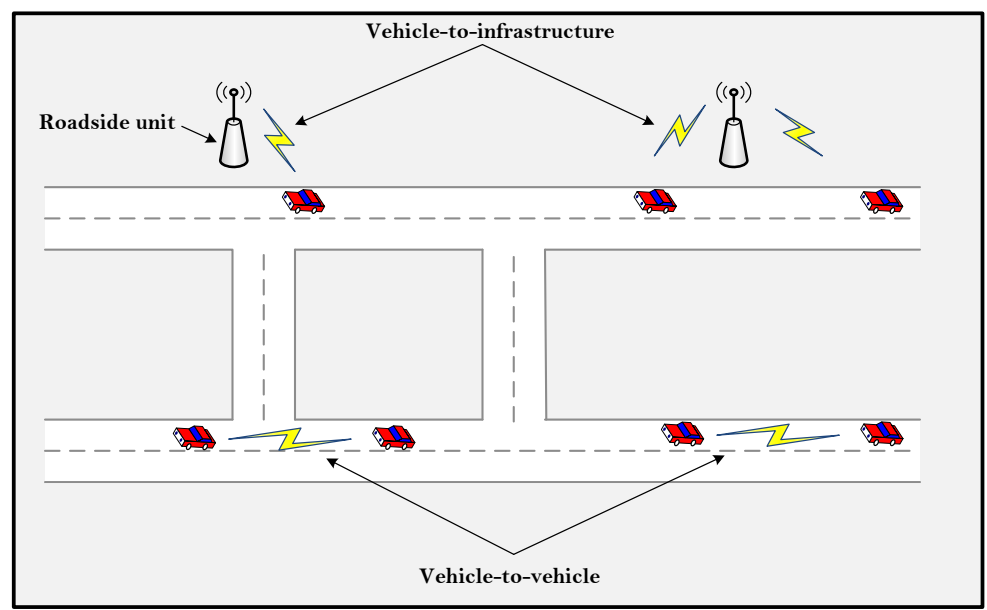

Figure 1.1: An illustration of V2V and V2I communication modes in VANETs.

\subsection{Motivation and Research Contributions}

Due to the nature of VANETs, the communication is affected by many factors. One of the factors that plays a significant role is the vehicle mobility. When vehicles move, the vehicle traffic density varies, the communication time between vehicles differs, and communication link disconnectivity can occur frequently. With the limited time offered for each vehicle to communicate, having a MAC protocol that provides a fair and effective sharing of radio resources while maintaining the required QoS is challenging but crucial.

A simple example of the mobility impact on VANET communication occurs when nearby vehicles move with different speeds. In the V2I mode, a vehicle with high speed can enter and leave the communication range of the RSU without getting much channel access. On the other hand, a low-speed vehicle can have a relatively long communication time with the RSU as shown in Figure $1.2(\mathrm{a})$, which indicates the problem of unfairness in the V2I communications. Karamad et. al. proposed a modified MAC version of the IEEE 802.11p to assure fairness in the V2I mode [3]. However, to the best of our knowledge, the 
problem of unfairness due to the different node speeds has not been identified in the V2V communication mode. For example, Figure 1.2(b) shows a simple case for three nodes communicating in the $\mathrm{V} 2 \mathrm{~V}$ mode. Node A moves with an average speed while node $\mathrm{B}$ moves with an extremely high speed. It can be seen that, after some time, node B will be out of the active communication range while A can still communicate with the sending node in the range. This shows the significant impact of node relative speed in V2V communications. An effective MAC protocol should provide priority to node B to transmit before it moves out of the communication range.

The IEEE draft standard 802.11p [4, included in the wireless access in vehicular environment (WAVE) stack, is the only standard for MAC in VANET. Since the 802.11p uses the basic mechanism of the Distributed Coordination Function (DCF) that was originally designed for low mobility networks, it does not operate efficiently for high mobility communication scenarios in VANETs. Moreover, the IEEE 802.11p standard does not consider the mobility impact at the medium access level. Extensive studies in the literature show that IEEE $802.11 \mathrm{p}$ does not provide the required performance in terms of packet delivery ratio (PDR), throughput, and fairness $[3][5][6][7][8]$. The protocol suffers from severe performance degradation with high node density, high data traffic, high speeds, and network partitions. Without considering the preceding factors in the MAC operation, QoS provisioning for both safety and multimedia applications is difficult to achieve.

The objective of this research is to address the above problems and develop an effective MAC protocol that provides better PDR, throughput, and fairness in a multihop infrastructureless vehicular networks. In this thesis, we provide a comprehensive performance evaluation based on a mobility and performance metrics to study the impact of mobility on the IEEE 802.11p MAC. The study focuses on the relation between mobility factors 


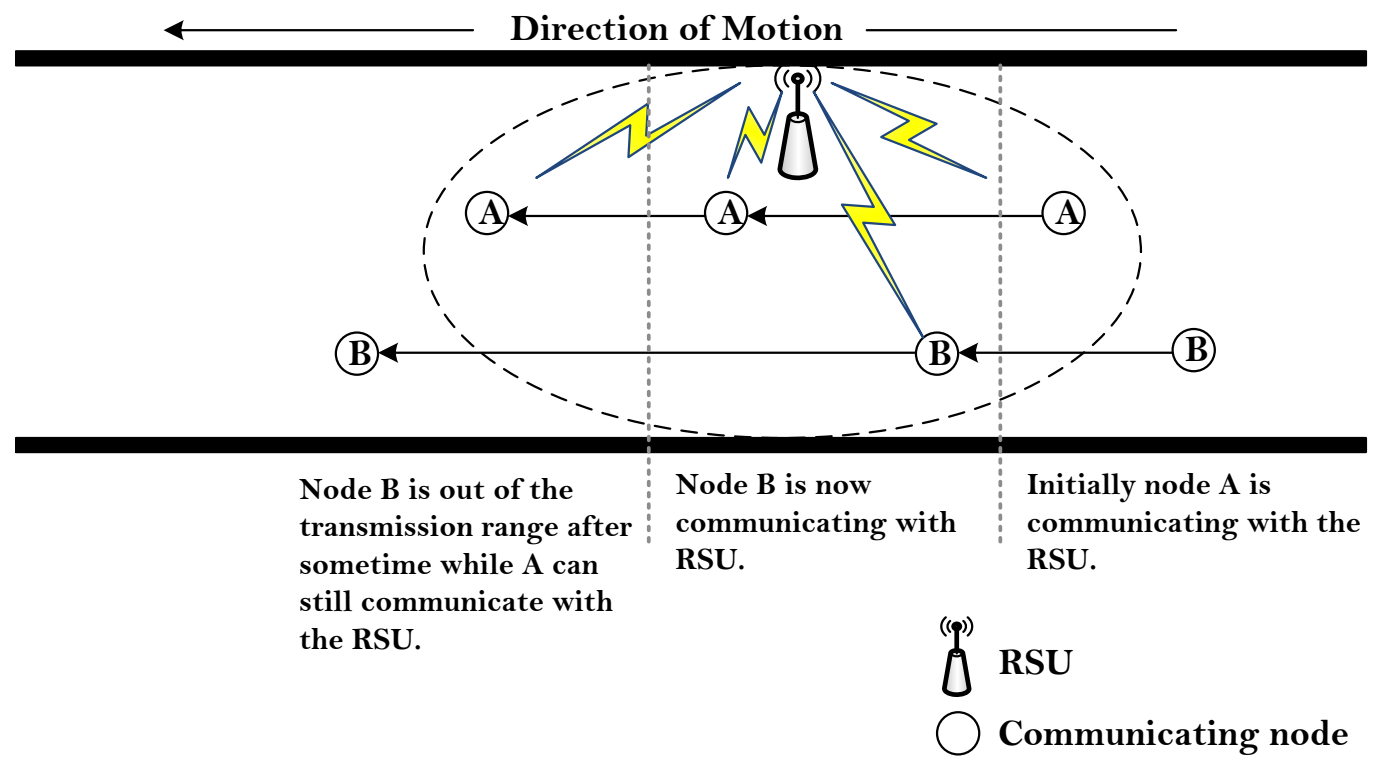

(a)

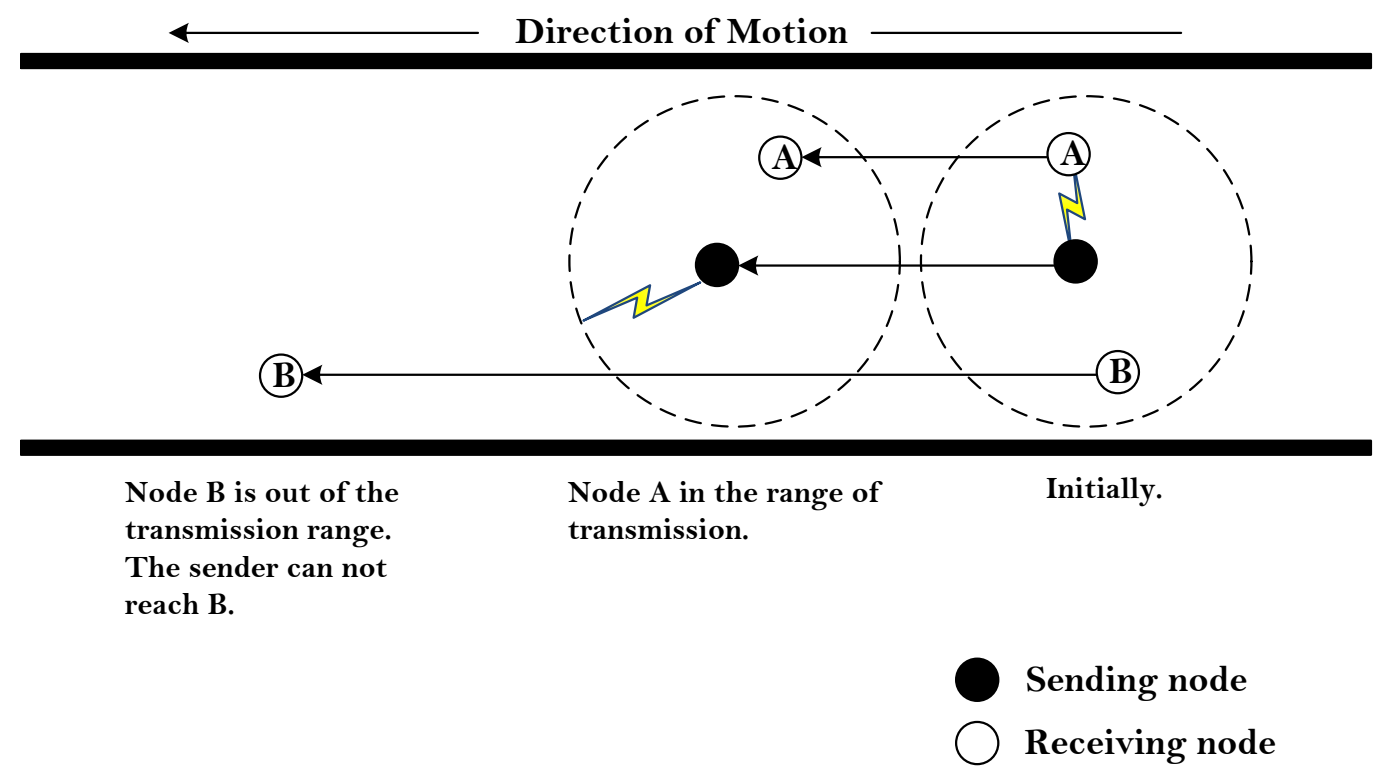

(b)

Figure 1.2: The problem of unfairness in channel access due to nodes having different speeds. 
and the respective behavior of the MAC protocol. Our study shows that relative speed of the communicating vehicles has a significant impact on the channel access time provided at the MAC layer. To the best of our knowledge, we are the first to identify the problem of unfairness due to the relative speed for the $\mathrm{V} 2 \mathrm{~V}$ communications at the MAC level.

Moreover, we propose two dynamic priority channel access schemes that are based on the IEEE 802.11 DCF function. Both schemes provide adaptivity to the vehicular mobility. To that end, vehicles are assigned different access priorities according to their speeds and neighbor node densities. It is shown that both protocols provide better performance than the IEEE 802.11p standard in terms of PDR and average number of retransmissions per packet.

\subsection{Thesis Organization}

The remainder of this thesis is organized as follows. Chapter 2 presents a literature review of the MAC protocols for VANETs, and the recent related work. Chapter 3 describes the system model considered in our research. In addition, it presents the proposed priority access schemes. Chapter 4 presents the performance evaluation of the current IEEE $802.11 \mathrm{p}$ standard. Moreover, the mobility impact is thoroughly studied according to mobility met-

rics, and the performance of the mobility adaptive MAC protocols is presented. Finally, Chapter 5 gives concluding remarks of our work and outlines the possible future work. 


\section{Chapter 2}

\section{Literature Review and Related Work}

In this literature review, the main medium access control (MAC) protocols that are proposed for VANETs are reviewed in such a way that each protocol is briefly explained, and the issues it addresses are identified. In this chapter, we discuss the essential background knowledge about VANET MAC and explain some related work research.

\subsection{MAC Protocols for Vehicular Ad Hoc Networks}

MAC protocols are considered crucial and important when measuring the network performance. The importance of MAC protocols in defining how each node shares the limited bandwidth in the network increases due to the special characteristics of the vehicular networks. Both high speed and fast topology changes make the process of sharing the bandwidth more difficult.

MAC protocols can be classified into two main types, centralized and decentralized. However, in VANETs, due to the lack of a central coordinator, distributed MAC protocols 
are expected to provide a reliable communication even though some VANET applications interact with infrastructure units, e.g. roadside units (RSUs) [9]. The majority of the protocols discussed in the literature are distributed.

For VANET MAC, random access protocols are extensively researched. In random access protocols, the nodes contend to access the medium and should be aware of the collisions. On the other hand, contention-free protocols, e.g., TDMA, CDMA, FDMA, determine which node should have access to the medium without any contention. There are some protocols that utilize the medium access by embedding the principle of schedulebased MAC. For example, ADHOC-MAC uses a dynamic TDMA mechanism [10].

Historically, ALOHA [1] is the base of random access protocols. The idea of ALOHA is basically that nodes send whenever they have packets to transmit. Based on ALOHA, slotted ALOHA (S-ALOHA) [1] provides a better medium access mechanism by dividing the time into slots, and a node only transmits at the beginning of a time slot. While ALOHA and S-ALOHA allow nodes to access the medium whenever they have packets to send, carrier sense multiple access (CSMA) [1] protocols allow a node to send only if the medium is not busy. Thus, the node checks the status of the channel before transmitting, and if the channel is busy, it backs off for a random amount of time; otherwise, it transmits. CSMA with collision detection (CSMA/CD) [1] and CSMA with collision avoidance (CSMA/CA) [1] are both inherited from the original CSMA protocol. However, the latter is the one that is applicable in wireless networks. As Section 2.4 shows, several protocols for medium access in VANETs are based on the CSMA mechanism such as IEEE 802.11 and its derivatives. 


\subsection{Quality-of-Service Metrics}

The performance of any MAC protocol is measured through certain metrics that are specified for a certain application. For example, some protocols are intended to increase the capacity and maintaining the delay at specific values, while other applications require delay to be minimized and scarifying some of the capacity for the transmission. In vehicular networks, depending on the application, certain QoS measures should be met. Generally, the following performance metrics should be considered by VANET MAC protocols:

Packet Delivery Ratio - Usually, packet delivery ratio (PDR) requirement depends on the type of the application. The PDR should be larger than a certain threshold to provide a specific service. To achieve a good PDR that satisfies certain QoS, the hidden node problem, which causes unexpected collisions, should be addressed. Section 2.3.4 explains the hidden and exposed node problems. To achieve a desired PDR, two factors can be dealt with at the MAC level. They are collisions (which occur due to the hidden node problem) and transmission interference. Some performance metrics do not consider PDR. Instead, they consider the probability of packet reception or, alternatively, the probability of reception failure. Generally, PDR or packet loss rate (PLR), which is the complement of the PDR, is used as a measure of the transmission reliability of the MAC protocol. In some vehicular network applications, e.g., safety messages in safety applications, the packet delivery rate should be very high (>99\%).

Delay - An important requirement for vehicular communications is that a message should be delivered within a certain time. This time is known as communication delay bound, and can be defined as the maximum time duration between the generation and the successful reception of that message. In many cases, especially for safety applications 
in vehicular networks, if the message is delivered after the delay bound, it is considered useless. For example, in [1], it is mentioned that accident information should be delivered in a maximum of half a second to all desired destinations. Other specification requires a maximum of $100 \mathrm{~ms}$ or $50 \mathrm{~ms}$ delay depending on the application. Consider the scenario where two vehicles are moving in opposite directions, the delay of transmission in this case should be very small. In such a case, the delay should be bounded by a limit that is called deadline. After the reception deadline, the message is not considered fresh anymore.

Channel Busy Time - As mentioned in Section 2.1, when a node is willing to transmit using a CSMA protocol, it may find the channel busy and backoff for a certain amount of time. This time is the channel busy time. Reducing the channel busy time results in better channel utilization. For vehicular networks, $\mathrm{Xu}$ et al. [2] have defined the channel busy time (CBT) for safety message communication in the dedicated short range communications (DSRC) spectrum range (DSRC will be discussed later in Section 2.4). The control channel is monitored for a certain time $T_{i n v}$. During this monitoring time, the channel might be busy for some time, due to the transmission of other safety messages that might be delivered successfully or not. The transmission is assumed for a randomly chosen node and its neighbors who are located in the interference range of that node. If the total time of the transmission period is denoted by $T_{\text {total }}$, then the channel busy time can be defined mathematically as

$$
C B T=\frac{T_{t o t a l}}{T_{i n v}}
$$

Fairness - At the MAC level, if the probability of transmission from each node that is transmitting using the same MAC protocol is equal, then the protocol is considered 
fair. However in vehicular networks, due to the high mobility and differences of speed, fairness is difficult to achieve. Therefore, a certain level fairness is usually defined as a goal. Although it is difficult to achieve complete fairness, it is preferred to allow a tradeoff between fairness and other QoS metrics in order to achieve better overall QoS in certain applications.

\subsection{Challenges and Issues in VANET Environment}

There are several issues and difficulties that should be considered when designing a MAC protocol for VANETs. Some of these problems are classic and exist in other networks, e.g., hidden and exposed node problems, and others exist only in vehicular networks.

\subsubsection{High Speed and Frequent Topology Changes}

In vehicular networks, the vehicles move very fast on road. This causes frequent changes in the topology of the network. However, due to road geometry, the directions of the vehicles can be predicted to a certain extent. This issue should be handled carefully by the MAC protocol. For example, two nodes can communicate if they are in the transmission range of each other. If one node moves very fast, it will be out of the other node's range before completing the transmission. Due to high node density and high speed, the system performance can degrade dramatically. Another example is when vehicles move with speed of $120 \mathrm{~km}$ or even $150 \mathrm{~km}$ per hour, the probability of having frequent link disconnections increases. Therefore, the MAC protocol design should address mobility issues and estimate accurately the condition of the highly dynamic channel. 


\subsubsection{Lack of central coordination}

As mentioned precedently, vehicular networks are considered a special case of MANETs with high speed. Therefore, it is difficult for a centralized MAC protocol to coordinate the medium access. Currently, distributed MAC protocols are proposed to enhance the performance of vehicular networks even though some applications may involve communication with roadside units. In those cases, where central coordination is used partially or completely, the network generally does not operate in an ad hoc mode. However, the coordinator station can be considered on the other hand as a node that operates in ad hoc mode similar to any other vehicles moving on the road. As mentioned in Section 2.1, there are some protocols that are schedule-based, e.g., ADHOC MAC uses TDMA [10]. Those protocols are mainly intended to work in a centralized mode, which makes it difficult to apply them to vehicular networks without major changes [11].

\subsubsection{Scalability}

In a distributed system that has very high mobility such as in vehicular networks, changes of network size should be handled carefully. It is normal to have a situation where vehicle density is near the average. However, the vehicle density can suddenly grow significantly and becomes very large in a road segment. Operability in both sparse and high node density situations is very important for MAC protocols. An effective MAC protocol should be adaptive to various network information load and vehicle density. In this context, scalability can be defined as the ability to accept an increase in the number of nodes or elements in the network without suffering a noticeable decrement in performance or

a complexity increment [12]. Vehicular networks can be considered a typical example 
where scalability is required. Several studies on performance evaluations, some of which are mentioned in Section 2.4.3, show that some protocols do not work properly in a high node density and/or when the network is highly loaded with teletraffic. In such cases, the network may not provide the desired performance unless the MAC protocol is designed to address this issue.

\subsubsection{Hidden and Exposed Node Problems}

One of the classical problems in distributed MAC is the hidden node problem. In the situation in Figure 2.1(a), node A is transmitting to node B; At the same time node C, that can not detect the transmission from node A, wants to initiate a transmission to the same receiver node, $\mathrm{B}$. If $\mathrm{C}$ initiates the transmission, a collision occurs. This happens because both nodes $\mathrm{A}$ and $\mathrm{C}$ are out of each other's transmission range, while node $\mathrm{B}$ is placed in the transmission range of both A and C. In VANETs, due to the high speed mobility in vehicular networks, the hidden node problem is expected to happen more frequently.

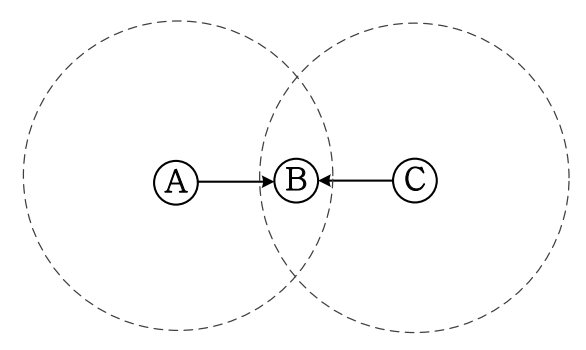

(a)

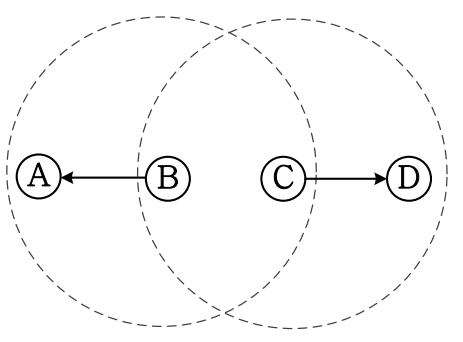

(b)

Figure 2.1: Hidden and exposed node problems. 
Another problem is the exposed node problem. In Figure 2.1(b), node B is transmitting to node $\mathrm{A}$. However, node $\mathrm{C}$, which is located in the transmission range of node $\mathrm{B}$, wants to initiate a transmission, but it hears node B transmitting. Thus, C will not transmit in order to avoid collision.

The hidden node problem can be solved by using the request-to-send/clear-to-send (RTS/CTS) handshaking as shown in Figure 2.2. Node A, which wants to transmit to node B, sends an RTS packet to inform node B that it wants to transmit. Node B responds to this request by broadcasting a CTS message to all the neighbors in its transmission range. Every node that hears the CTS should not start any transmission, especially the hidden node, $\mathrm{C}$ in this example.

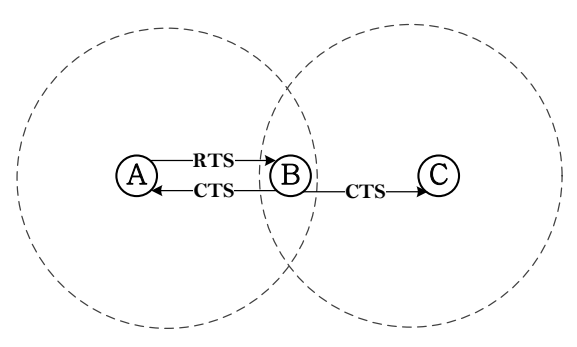

Figure 2.2: Solving hidden node problem using the RTS/CTS handshaking.

\subsection{IEEE 802.11 based protocols}

\subsubsection{IEEE 802.11 Standard}

IEEE 802.11 [13] is a communication standard for wireless networks. The 802.11 works in two modes; centralized and decentralized. The basic group of 802.11 nodes communicate with an access point (AP) is called Basic Service Set (BSS). The BSS allows nodes to 
communicate with the AP and gain access to its services after having some authentication, association, and multiple handshaking steps. On the other hand, the ad hoc mode allows the nodes to communicate with each other without any infrastructure and is called Independent BSS (IBSS). In vehicular networks, both modes are adopted with several modifications. Moreover, the IEEE 802.11 standard is often suggested for the implementation in vehicular networks due to the wide availability.

In IEEE 802.11, there are two methods to access the medium. Distributed Coordination Function (DCF), based on CSMA/CA, is used to coordinate the medium access in the ad hoc mode. The other function is point coordination function (PCF) which is used to control the medium access in a centralized mode. However, the majority of the protocols proposed for vehicular networks require operating in an ad hoc mode especially for V2V communications. On the other hand, if the MAC protocol involves infrastructure units such as RSUs or uses a virtual grouping mechanism, then a PCF or PCF-like function can be used for access coordination.

One of the functions used to coordinate channel access and guarantee QoS requirements is the Enhanced Distributed Channel Access (EDCA), which is used in the IEEE 802.11e [14], and is considered an enhanced version of the 802.11 DCF. The EDCA is based on CSMA/CA. The main feature of the EDCA is that, when the channel is busy, the back-off mechanism differs. The back-off procedure is as follows. A node that will transmit senses the channel first, and if the channel is busy, it will choose a back-off time sampled from a uniform distribution $[0, \mathrm{CW}](\mathrm{CW}$ is the contention window size). If the channel is free, the back-off time will be decreased. Otherwise it will be doubled. The node will transmit when the back-off value reaches 0. The IEEE 802.11e prioritizes messages by providing different Traffic Categories (TC) that are also called Access Categories (ACs). 


\subsubsection{IEEE 802.11p, WAVE and DSRC}

In the United States, the Federal Communication Commission (FCC) has allocated 75 $\mathrm{MHz}$ of the Dedicated Short Range Communications (DSRC) spectrum at the licensed band of $5.9 \mathrm{GHz}$, specifically in the range $5.850-5.925 \mathrm{GHz}$, for vehicular communications. The major goal is to provide safety for lives and enhance vehicle traffic flow. The DSRC spectrum consists of 7 channels, each one is $10 \mathrm{MHz}$ wide. Figure 2.3 shows these seven channels. Channel 178 or control channel $(\mathrm{CCH})$ is restricted for safety communications. The two channels at the edges of the spectrum are reserved for future applications. The remaining four channels are used for other services such as nonsafety applications. It should be noticed that, even though there are seven channels allocated for transmission, single radio device cannot transmit in more than one channel simultaneously, and this raises the need to efficient channel access coordination.

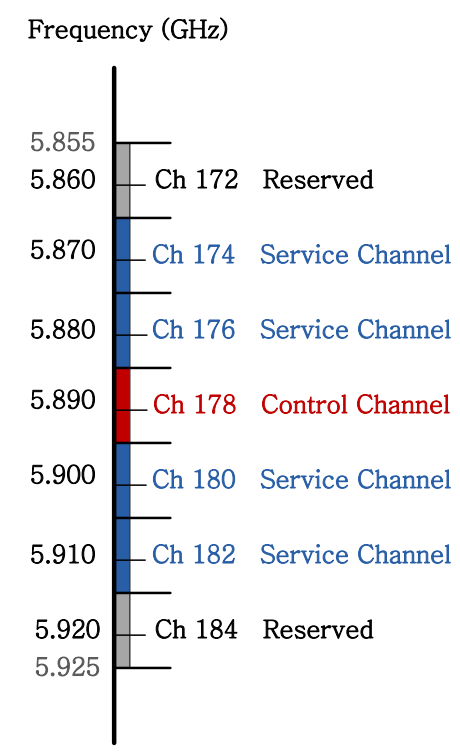

Figure 2.3: DSRC spectrum allocation by FCC. 
To work in the DSRC spectrum range, the IEEE 802.11 devices should operate in Wireless Access in Vehicular Networks (WAVE) mode or IEEE 802.11p [4]. IEEE 802.11p is an amendment for the IEEE 802.11 standard to make it suitable for an intelligent transportation systems (ITS) environment. Just like IEEE 802.11, IEEE 802.11p defines the MAC and PHY layers of the WAVE protocol stack. The upper level layers are defined in the IEEE 1609 [15] family of standards. As shown in Figure 2.4. IEEE 1609 is divided into four standards:

1. IEEE P1609.1 - Standard for WAVE - Resource Manager;

2. IEEE P1609.2 - Standard for WAVE - Security Services for Applications and Management Messages;

3. IEEE P1609.3 - Standard for WAVE - Networking Services;

4. IEEE P1609.4 (802.11p) - Standard for WAVE - Multi-Channel Operations.

For the PHY level, which is not the focus of our work, the IEEE 802.11p is required to provide communication with minimal modification of the 802.11 PHY layer. The standard is mainly based on the 802.11a-like PHY layer [9].

To work in a vehicular environment, the 802.11p MAC should simplify the BSS operations and reduce the amount of the overhead needed to establish a communication link. Thus, WAVE mode stations operate in the same channel and communicate immediately without wasting time in association when joining the BSS. The joining process is done using a wildcard Basic Service Set Identification (BSSID) which is the name of the BSS at the MAC layer. This new BSS mode is known as WAVE Basic Service Set (WBSS) in which authentication and association are not required. Instead, a station can join the 
WBSS according to the WAVE advertisement or announcement. More information about WBSS is given in [4]. The IEEE 802.11p is supposed to provide prioritized channel access through the use of the EDCA by providing different Access Categories (ACs). The ACs ranges from 0 to 3 , where $\mathrm{AC}_{0}$ and $\mathrm{AC}_{3}$ refer to the lowest and highest priority, respectively.

\begin{tabular}{|c|c|c|c|}
\hline Upper layers & $\begin{array}{l}\text { IEEE P1609.1 } \\
\quad+\text { others }\end{array}$ & & \\
\hline $\begin{array}{l}\text { Networking } \\
\text { Services }\end{array}$ & IEEE P1609.3 & IEEE P1609.2 & $\begin{array}{l}\text { Security } \\
\text { Services }\end{array}$ \\
\hline MAC \& PHY & $\begin{array}{l}\text { IEEE P1609.4 } \\
802.11 \mathrm{p}\end{array}$ & & \\
\hline
\end{tabular}

Figure 2.4: The IEEE 1609 standards.

\subsubsection{Related Work Review}

The IEEE 802.11p has been extensively studied [5] [6] [8] [16]. However, to the best of our knowledge, there is no comprehensive evaluation that reflects the impact of mobility on the IEEE 802.11p MAC protocol performance, especially for the V2V communications. Moreover, very little work has been done on enhancing the performance of IEEE 802.11p via adaptation to the mobility factors.

The IEEE 802.11p is meant to provide reliable and efficient MAC for the high speed vehicular environment. In the literature, researchers are continuing to investigate and study the performance of the 802.11p, 802.11- and 802.11p-based MAC protocols, and study their suitability for vehicular networks. It is known that $802.11 \mathrm{MAC}$ is designed 
for low mobility and has some limitations especially in a high density scenario. Since the IEEE 802.11p is based on the original IEEE 802.11, it is normal for it or any other protocol based on 802.11p to inherit those limitations. In [5], the authors have studied the saturated performance of 802.11 MAC in a single-hop network. The study shows the delay requirement, which is below $100 \mathrm{~ms}$, is satisfied while the PDR decreases dramatically when the number of nodes increases. The authors suggest that the reason for the failure on achieving the desired PDR rate (more than 99 per cent) is the high collisions due to the fixed short back-off window and hidden node problem. Several enhancements at the 802.11 MAC level are suggested such as repetition mechanisms, adaptive backoff, and dynamic adjustments of the contention window size.

The 802.11 Wireless LAN (WLAN) performance has been studied over different scenarios and with a reactive routing protocol in [17]. The performance indexes used to test the network performance are throughput and the signal to noise ratio (SNR). Experimental results show that the 802.11 throughput decreases dramatically in a high node density and large network. The recommended solutions are to modify the back-off mechanism, using a super frame structure, and using different inter-frame spacing system for different service priority. In [18], the simulation results of $802.11 \mathrm{p}$ MAC in a highway scenario show that some nodes are forced to drop over $80 \%$ of its time-critical messages due to the long channel busy time. This can lead to serious failure in delivering safety messages.

In [7], simulation results of the IEEE 802.11p standard show that the fixation of the back-off window size does not guarantee the desired throughput in vehicular networks. Two algorithms for a dynamic back-off window size are proposed. In the centralized approach, the base station is assumed to know the number of transmitting nodes and, accordingly, computes the optimal transmission probability. In the distributed algorithm, local medium 
information is needed for the vehicle to select the back-off time. Therefore, the number of current users is estimated by observing the current contending nodes. Both algorithms improve the performance over the 802.11 static back-off mechanism. However, the enhancement proposed is used for the V2I communication mode and even though the throughput has been improved, the computed contention window sizes are very large and can cause very long delay in transmission. Similar simulation results supported with analytical means show that the IEEE 802.11p suffers from an undesired decrease in throughput and increase in delay in high density scenarios [6]. The study evaluates the collision probability, throughput, and delay in the performance evaluation, and focuses on WAVE message prioritization using the EDCA mechanism. It is mentioned that increasing the $C W$ size in order to reduce the probability of collisions causes the throughput to decrease. An alternative is to shape the traffic while maintaining the number of high priority packets at a certain level. To achieve better performance, the author suggests using a re-evaluation mechanism for messages to reduce the number of high priority messages continuously. A similar study on the factors affecting the performance of the ad hoc networks gives similar suggestions [19]. It is shown that increasing the network size, while maintaining the traffic load, leads to increased throughput. To apply this in vehicular networks, the number of potential neighbors should be considered. Aiming at multi-hop communications, Stibor et al. [16] evaluate the number of potential communication partners and the maximum communication time for vehicular ad hoc networks using the IEEE 802.11p standard. The simulation is done for a highway scenario, and the results show that the number of neighbors varies and leads to different communications time between vehicles. It is shown that the number of neighbors should be used as an input parameter in multi-hop communications.

Other issues that the IEEE 802.11p should address are mentioned in [20]. To make 
the IEEE $802.11 \mathrm{p}$ work efficiently, some challenges should be addressed. These challenges are the stateless channel access, caching for handoff, and opportunistic frame scheduling. By operating in the WAVE mode, WBSS providers (e.g., APs) cannot keep track of the WBSS users due to the absence of authentication and association in the WBSS. Moreover, when a WBSS user moves out of a WBSS provider's range, the WBSS provider does not know that the WBSS user is not in its range anymore because there is no de-association process. The mobility of a WBSS user leads to its communication with multiple WBSS providers. Therefore, supporting fast handoff should be considered. In [21], a simulation framework that includes handoff mechanisms for the IEEE 802.11p is developed to study the behavior of handovers for the V2I communications. A solicitation-based IEEE 802.11p MAC protocol is proposed for roadside to vehicle communication [20]. The solicitation MAC protocol is based on a wired backbone for the WBSS providers, and introduces two new concepts. A new operation mode called WBSS user initiation mode (W-UIM), in which the WBSS user opportunistically solicits data frames for itself. A WAVE-poll frame is used to request the transmission from a WBSS provider. The other concept is that the adjacent WBSSs are virtually grouped and denoted as a WBSS-area.

Karamad and Ashtiani propose a modified MAC protocol based on 802.11 DCF to assure fairness in the V2I communications [3]. The idea is to adapt the minimum contention window of every node to its speed. To do that, the scheme modifies the probability of transmission according to the node speed. Moreover, the probability of transmission changes according to the adjustment of the contention window size. In this way, a relation between the speed and the window size is obtained. Analytical approximation for this relation is validated by simulation and the protocol can handle the intuitive unfairness problem.

A study in [8] showes that node speed is not a significant factor in terms of aggregate 
throughput, average delay, and packet loss rate. However, traffic load is shown to be a significant factor that affects the performance. From the simulation results, when the vehicle density increases, both aggregate throughput and average delay increases while the delay requirement is met. However, the simulation is done only for a one-lane road scenario.

\subsection{Virtual Grouping MAC}

\subsubsection{Cluster-based MAC}

Clustering has been proposed for ad hoc networks. Clustering schemes can be used to manage the mobility and the quick changes in the network topology. In a vehicular environment, since vehicles sometimes move with similar speeds and in the same direction, dividing them into groups through clustering can help controlling the medium access. Clustering is basically used by routing in mobile ad hoc networks to handle the problem of flooding. However, in the medium access layer, clustering is used to handle the hidden node problem, provide better scalability, reduce the number of interfering nodes, limit the area of message dissemination, and provide fair access to the medium [22].

By using clustering techniques, an ad hoc network can be handled by a centralized controller. This occurs by virtually grouping the mobile nodes into clusters. In each cluster, a Clusterhead $(\mathrm{CH})$ is elected to act as a central controller that coordinates intra-cluster transmission. The other nodes in the cluster are considered clustermembers, which are normal nodes, or cluster gateways. Figure 3.3 shows an example of a clustering structure. More information about clustering and cluster formation algorithms can be found in [23]. 


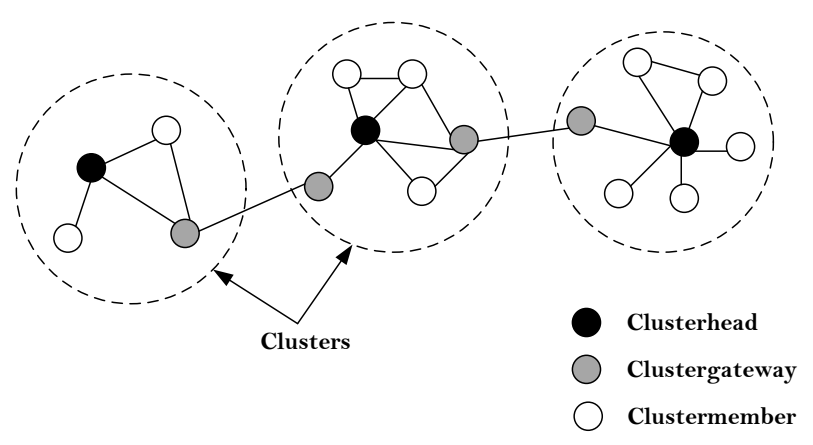

Figure 2.5: Clustering structure example.

For vehicular networks, research on cluster-based MAC focuses on clustering formulation algorithms, improving the medium access through clustering, minimizing the cost by using a fewer number of transceivers, and avoiding the inter-cluster interference. The authors in 22 proposes a clustering medium access mechanism based on TDMA. The main function of the clusterhead is to manage the bandwidth assignment in the cluster. Through simulation, the protocol is shown to be stable except for a high dense traffic situation such as in rush hours. Su and Zhang propose a clustering-based multichannel MAC scheme in [24]. Their scheme integrates clustering with contention-free and contention-based MAC protocols. Each vehicle is supposed to use two transceivers that operate simultaneously. The clusterhead of each cluster has three functions to perform: 1) collection and delivery of safety messages within a cluster, 2) forwarding the safety messages to the neighboring clusters, and 3) coordinating the channel access for local clustermembers with non-realtime communication flows. The contention-free MAC for communication within a cluster is TDMA, while the IEEE 802.11 is used for communications between clusterheads. The protocol is designed to provide QoS for the realtime data, e.g. safety messages, and provide an increased throughput for non-realtime data for a V2V communication scenario. 
Based on the analytical model and the comparison with the IEEE 802.11 and V2V dynamic channel assignment (DCA) 24] through simulations, the proposed MAC scheme achieves both timely delivery of safety messages and high throughput for non-realtime traffic. A similar idea is proposed in [25], but using only one DSRC transceiver in each vehicle. The proposed protocol uses a clusterhead for the intra-cluster communication and cluster forwarder for the communication with backward clusters. Inter-cluster interference, that occurs to clusters close to each others as in Figure 2.6 or due to clusters overlapping, is handled with a simple algorithm.

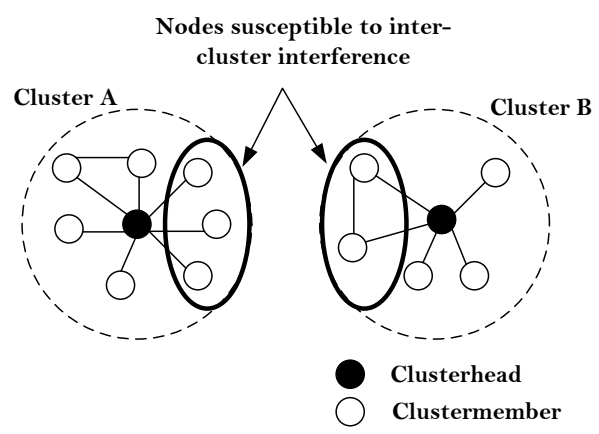

Figure 2.6: Example of inter-cluster interference.

\subsubsection{Space Division Multiple Access (SDMA)}

Space Division Multiple Access (SDMA) is proposed for inter-vehicular networks in [26] [27] [28].

The protocol is only theoretically investigated. The SDMA introduces a new concept of partitioning the geographical area into multiple divisions. Each of these divisions is mapped to a certain channel to achieve desired performance. To perform the mapping process accurately, the precise positions of the vehicles are assumed to be known. This can be done by using GPS devices. 
The SDMA aims to reduce access collisions, and increase channel reusability. However, many issues and challenges exist for the SDMA in vehicular networks. In [29], the suitability of SDMA in VANETs is investigated. For example, the partitioning process of the road depends on the road map. It is shown that SDMA is suitable for a highway scenario, but there are other scenarios that should be dealt with such as meshed road scenario [29]. Therefore, the SDMA should handle such a challenge when performing the partitioning and mapping processes.

\subsubsection{Token-Ring based MAC}

In [30], a wireless token ring protocol (WTRP) is proposed for intelligent transportation systems. The protocol is evaluated and implemented on top of the IEEE 802.11 DCF. No mobility consideration is mentioned in the work. In [31], an extension of the WRTP with three implementations is proposed. The implementations are a simulator implementation, an application layer implementation that is platform-independent, and a kernel implementation which is a Linux link layer module built on top of the IEEE 802.11 DCF. In order to achieve reliable and fast communication for safety applications as well as QoS guarantee for data services, an overlay token ring protocol (OTRP) is proposed in [32]. The OTRP minimizes the number of possible collisions, and transmits safety messages with high probability and low delay. Another advantage is the feasibility of implementation without complex hardware requirements. 


\subsection{Other protocols}

\subsubsection{ADHOC MAC}

ADHOC MAC [10] is a MAC protocol developed under the CarTalk2000 project to support inter-vehicular communications. ADHOC MAC uses a dynamic TDMA mechanism with slotted frame structure that is independent from the physical layer. The protocol is based on Reliable R-ALOHA (RR-ALOHA), which is an extended version of Reservation ALOHA (R-ALOHA). R-ALOHA is capable of coordinating the channel access in a centralized mode. RR-ALOHA aims to do the same thing, but in a fully distributed mode. The protocol is supposed to deal with the hidden and exposed node problems, provide a reliable single-hop broadcast service, and reserve additional bandwidth and QoS for real-time traffic as needed in the applications. An advantage of ADHOC MAC is that it can be adapted to work with the 802.11 physical layer by providing a frame structure.

In [1] [5], several issues regarding ADHOC MAC are discussed. It is mentioned that the minimum time needed to successfully obtain the basic channel is greater than $200 \mathrm{~ms}$ in a static scenario. Moreover, several factors such as mobility and dense traffic may cause more latency in allocating and releasing slots. Another issue is the number of frame slots that should be optimized. It is known that frame size is related to other parameters such as network capacity. Thus, the number of vehicles in the same communication range must not exceed the number of slots in each time frame. In comparison with the IEEE 802.11, ADHOC MAC is not utilizing the medium efficiently, and does not handle high mobility

as the 802.11 does. Further information about ADHOC MAC performance can be found in [17]. 


\subsubsection{Directional Antenna-Based MAC}

In VANETs, vehicles move according to road geometry. Therefore, the transmission of information, depending on the application, should be done in specific directions. For example, for a safety application that should warn vehicles of sudden breaking, the information should be send to the vehicles that follow the vehicle with the breaking action. Other vehicles in front of the broadcasting vehicle are probably not affected by this breaking action. This can be done by using directional antenna-based MAC protocols. For more information about MAC protocols with directional antennas, [33] is considered a good reference that provides a classification of MAC protocols with directional antennas, and discusses the challenges in their design.

Directional antennas are used to overcome some problems such as interference, hidden node and exposed node problems. In addition, increased transmission range, and reuse of channels are the main benefits brought by those protocols. Ideally, the transmission range is divided into $x$ non-overlapping transmission angles each having a degree of $360 / x$. It is known that increasing $x$ results in smaller antennas angles and narrower transmission range. One requirement in directional antenna protocols is the knowledge of transmitter's and the receiver's active antennas during the transmission. This can be done by having neighbors' location information using a GPS, by neighbors location estimation, or by continuous neighborhood discovery. While the use of GPS devices incur extra cost and has some limitations, location estimation methods such as Angle of Arrival (AoA) may cause some errors [33. On the other hand, continuous neighbor scanning are used in several protocols such as [34]. Furthermore, directional antennas can be classified as traditional directional or smart antennas [33]. 
For vehicular networks, several MAC protocols adopt directional antennas and achieve network performance improvement as in [35] [36] [37]. However, investigations of the performance and the suitability for VANETs show that, even though directional MAC protocols improve the performance by reducing collisions and increasing channel reusability, complexity and difficulties of providing practical implementation are the major issues of directional MAC in VANETs. More information regarding directional antenna MAC performance can be found in [38].

\subsubsection{Repetition-based MAC}

The idea of repetition-based MAC is proposed by Xu et al. in [2] [39]. The aim of the protocol is to deliver safety messages in an ad hoc mode with high reliability and low delay. In [2], several random access protocols for medium access which are compatible with the DSRC multichannel architecture are discussed and used in the design. The main advantage of the repetition is to have a better probability of reception. However, undesirable amount of reception may cause severe consequences. The main idea is based on repetition. The lifetime of a message is divided into several slots based on its useful lifetime and the transmission time. A variable number of slots are randomly chosen for repetition. A reception of at least one packet means that the message is successfully received within the limited delay. Otherwise, a failure of reception occurs. The main issue here is how to find the number of slots to be selected for repetition. Figure 2.7 illustrates the idea of repetition.

The repetition may overcome the collision problem, and the main advantage of this design is its simplicity. In this protocol, a MAC extension layer handles the generation and removal of repetitions. The MAC extension layer is between the logical link layer and 


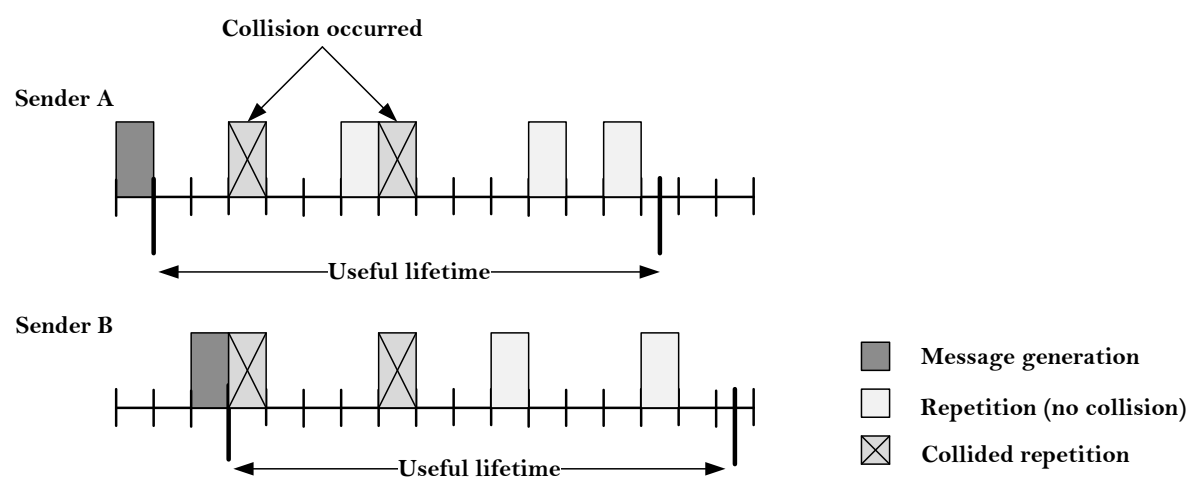

Figure 2.7: Illustration of the idea of repetition for two senders A and B.

the MAC layer.

Some proposals utilize optical orthogonal codes (OCC) to minimize the message loss probability, reach better probability of detection, and reduce the reception delay such as in [40]. The main idea is to use these codes to assure that a certain number of repetitions of every two nodes cause a collision. By knowing the number of collisions between the two nodes, it is obvious that using a larger number of repetitions will grantee a successful reception. The same concept is used in [41] to provide different QoS priority levels.

Another repetition based MAC is proposed in [42]. In this protocol, a distributed feedback mechanism is used to optimize the number of repetitions. The feedback mechanism is used to broadcast information regarding the transmission and reception of messages through the network. According to the broadcasted information, an algorithm based on index coding is used to minimize the number of transmissions. It is shown through simulations that the protocol results in a lower message loss probability compared with the previous repetition-based MAC protocols, but close results in terms of average delay. 


\subsubsection{Multichannel MAC}

It is known that inter-vehicle communication systems are proposed for safety applications and traffic enhancements. However, non-safety applications, or commercial applications, have been proposed in the literature to provide effective use of the DSRC spectrum [43] [44] 45]. The best solution to provide nonsafety realtime applications is through the multichannel communications. It has been explained in Section 2.4.2 that DSRC allows multiple channel communications by operating only one channel at a time. This is because there is only one radio used in DSRC. The difficult part of designing a multichannel MAC protocol is to provide the nonesafety communication while meeting the QoS of the safety communications. As discussed in Section 2.5.1, Su and Zhang propose a multichannel protocol that operates in two different radios simultaneously. This type of communication is considered a multichannel operating protocol, but it is also considered costly since each vehicle should be equipped with two transceivers.

Wang and Hassan propose a framework that performs periodic channel switching over DSRC to provide a concurrent safety and nonsafety applications in [43]. It is found that, during rush hours of traffic, the nonsafety applications can be extremely restricted to assure the QoS of the safety applications. An interesting conclusion is that using simple techniques can increase the commercial nonsafety applications opportunities. Moreover, the authors suggested the use of an adaptive scheme to perform dynamic adjustments to the control channel interval to support the switching between safety and commercial applications.

In [45], multichannel single radio MAC is proposed to provide V2V and V2I communications. The protocol aims at providing concurrent safety and commercial services. The work is first presented with the basic idea in [44]. However, in [45], the authors extend 
their work with proof of theorems of the design, and performance evaluation with the IEEE 802.11 DCF used in the ad hoc mode and PCF used in the centralized mode. The protocol is tested in three configurations: the DCF-only, the PCF-only in the hotspot area, and dedicated coordinating AP (DCAP) protocol configuration. The DCAP configuration is based on the DCF, PCF, and spatial division functions. These configurations are simulated using NS-2 in a four-lane highway scenario with high density of a vehicle flow. The DCAP configuration is shown to offer more consistent QoS than the other two configurations.

A cognitive MAC protocol for VANETs (CMV) based on cognitive radio management is proposed in [46]. In CMV, the protocol provides long-term and short term spectrum access which is applied in vehicular communications channels. The protocol is applied on DSRC channels. The cognitive radio management is used to improve the capacity

in long-term spectrum access. For short-term access, the wideband spectrum pooling is used. The protocol showed a significant improvement in throughput compared with other multichannel protocols.

\subsection{Comparison of the Existing Protocols}

MAC protocols are designed to achieve better medium access and overcome some obstacles in the system, such as the issues discussed in Section 2.3 for vehicular networks. To compare MAC protocols, certain criteria must be considered. Here, the protocols are compared qualitatively. The issues each protocol solved are emphasized, and the limitations are pointed out. Since vehicular networks have their special characteristics, till now, there is still no comprehensive solution that is suitable for all situations, scenarios, and QoS requirements. The IEEE $802.11 \mathrm{p}$ standard at the $\mathrm{MAC}$ is one of the most promising 
protocols due the popularity of the 802.11 protocol. The 802.11 p aims at providing a multichannel operation over DSRC with QoS support.

Since the $802.11 \mathrm{p}$ is amended from the original 802.11, it is obvious that it inherits its limitations. The 802.11p and 802.11p-basad protocols perform better in a low node density, and tend to provide unstable results in a highly loaded situation. The hidden node problem is addressed by the carrier sensing mechanism. However, throughput and PDR are highly affected by a high node density. A logical explanation of what happens in a highly traffic loaded scenario with an 802.11-based MAC protocol is that as the channel busy time becomes larger, nodes have to wait longer time before retransmission. Moreover, when a node retransmits during the busy time, the contention window size increases due to the back-off mechanism. Therefore, the number of nodes in the network is a significant factor that affects MAC performance. Prioritizing messages is shown to cause decrement of throughput when the number of high priority packets increases. The frequent link disconnections can be dealt with by evaluating network state, which is not included in the IEEE 802.11p standard. To achieve better evaluation of the network state, node speed can be used to predict the duration of transmission, and help to adapt the medium access protocol.

ADHOC MAC reduces collisions, handles hidden and exposed node problems, and provides a reliable single-hop broadcast service. It also reserves additional bandwidth and QoS for real-time traffic. However, the performance of ADHOC MAC degrades with high mobility and node density. Moreover, the number of vehicles in the same communication range is restricted by the number of slots in the time frame. ADHOC MAC is considered independent from the physical layer and can work with the 802.11 physical layer.

Transmitting at a specific direction is done by directional antennas-based MAC. This 
type of MAC is used to increase transmission range and channel reusability. In vehicular networks, directional MAC does not provide a feasible solution due to the complexity of implementation.

Clustering is used to handle mobility and frequent network topology changes. However, clustering is considered for average speed and similar direction mobility. Moreover, clustering provides better scalability, reduces interference between nodes in each cluster, limits the area for message dissemination, and achieves fair access to the medium. The problem with clustering is in deciding which node should be elected to be the clusterhead, and how to handle interference between nodes in clusters (intra-cluster interference) and among the clusters (inter-cluster interference).

The repetition based MAC protocols aim at a high probability of reception with low delay. A MAC extension layer is required to perform that. However, the number of repetitions and the generation process should be optimized. The SDMA and token ring based MAC protocols are based on grouping the nodes. While the SDMA aims at reducing access collisions and increasing channel reusability, it is only theoretically studied, and no feasible implementation exists. Similarly, token ring protocols reduce the number possible collisions, and deliver safety messages with a high probability within low delay. The advantage of token ring MAC protocols is that they can be implemented without high hardware complexity, but there are no practical implemetations for VANETs.

A multichannel solution that efficiently utilizes the bandwidth and meets the QoS requirements can be considered a total solution, but with a non-avoidable complexity. The existing multichannel protocols try to achieve the goal, but with ideas that are not feasible for implementation. 


\begin{tabular}{|c|c|c|}
\hline Protocol(s) & Pros. & Cons. \\
\hline 802.11- and 802.11p-based & $\begin{array}{l}\text { 1. Handles hidden node problem. } \\
\text { 2. Works in centralized and distributed } \\
\text { mode. } \\
\text { 3. Widely accepted. }\end{array}$ & $\begin{array}{l}\text { 1. Inefficient with loaded scenarios. } \\
\text { 2. Use a static back-off mechanism. } \\
\text { 3. No deterministic upper bound for chan- } \\
\text { nel access delay. }\end{array}$ \\
\hline ADHOC MAC & $\begin{array}{l}\text { 1. Avoids hidden terminal problem. } \\
\text { 2. Can be adapted to work with } 802.11 \\
\text { PHY by providing frame structure. } \\
\text { 3. Provides reliable one-hop transmission. }\end{array}$ & $\begin{array}{l}\text { 1. Limited number of nodes. } \\
\text { 2. Requires time synchronization. }\end{array}$ \\
\hline $\begin{array}{l}\text { Directional Antennas-based } \\
\text { MAC }\end{array}$ & $\begin{array}{l}\text { 1. Reduces collisions. } \\
\text { 2. Increases channel reusability. }\end{array}$ & 1. Complexity of implementation. \\
\hline Cluster-based MAC & $\begin{array}{l}\text { 1. Good solution for mobility and frequent } \\
\text { topology changes. } \\
\text { 2. Better scalability. } \\
\text { 3. Reduced Interference. } \\
\text { 4. Fair access. }\end{array}$ & $\begin{array}{l}\text { 1. Can be complex in implementation. } \\
\text { 2. Inter-cluster and intra-cluster interfer- } \\
\text { ence must be handled. }\end{array}$ \\
\hline Repetition-based MAC & 1. Better probability of reception. & $\begin{array}{l}\text { 1. Requires MAC extension layers to han- } \\
\text { dle the generation and removal of repeti- } \\
\text { tions. }\end{array}$ \\
\hline Multichannel MAC & 1. Utilization of the bandwidth. & 1. Complexity. \\
\hline SDMA & $\begin{array}{l}\text { 1. Reduce access collisions, and increase } \\
\text { channel reusability. }\end{array}$ & 1. No feasible implementation exists. \\
\hline Token ring MAC & $\begin{array}{l}\text { 1. Reduce the number possible collisions. } \\
\text { 2. Deliver safety messages with high prob- } \\
\text { ability within low delay. } 3 \text {. Can be imple- } \\
\text { mented without high hardware complexity. }\end{array}$ & $\begin{array}{l}\text { 1. No feasible implementation exists for } \\
\text { VANET. }\end{array}$ \\
\hline
\end{tabular}

Table 2.1: Comparison between the current VANET MAC protocols.

\subsection{Summary}

In this chapter, a literature review for MAC protocols in vehicular networks supported with related background is presented. First, the special characteristics of VANETs and their relation with medium access are discussed. Issues that should be addressed by VANET MAC are presented. After that, an overview of the existing MAC solutions for a vehicular environment are briefly introduced to provide a broad view of the existing solutions from the IEEE 802.11p standard, through virtual grouping mechanisms, multichannel utilization, and other enhancement techniques. Finally, a qualitative comparison of the existing protocols is provided. 


\section{Chapter 3}

\section{System Model and Mobility Adaptive Schemes}

In vehicular networks, with the existence of different traffic scenarios, different medium access techniques are adopted to provide better medium sharing in terms of efficiency and fairness. Until now, there is no MAC scheme that provides the desired performance in all communication scenarios. The main issues with VANETs are the mobility of vehicles and, in most cases, the fact of not having a fixed network infrastructure. Therefore, traffic velocities and, accordingly, their physical positions affect the performance of the communication protocol. Moreover, a major issue that should be solved in VANET MAC is the adaptation to fluctuations in vehicle traffic density.

The MAC scheme under consideration is a modified version of the IEEE 802.11 MAC. As mentioned in Chapter 2, the original 802.11p protocol performance has been extensively studied and several drawbacks have been identified [5, 17, 18, 7, 6]. Here, we are proposing two modified 802.11-based MAC schemes to provide priority channel access by adapting 
to mobility factors. In VANETs, nodes move with different speeds. Therefore, nodes with extreme velocity may enter and leave the range of communication in a very short time while other nodes with similar velocities have the opportunity to communicate for a longer time. Accordingly, a fair MAC scheme should provide priority channel access to the former class of vehicles. This problem can be clearly identified for a cluster of vehicles communicating in an ad hoc mode. Although virtual grouping mechanisms such as clustering are used to enhance communication stability, the cost of maintaining clusters in terms of overhead is generally high. Instead of that, we are using a periodic message broadcasting to exchange information among nodes and this is compatible with the VANET basic operation. Moreover, it is shown in [6] [7][16] that in dense traffic and with sudden changes in the number of communication partners, the performance of the 802.11 MAC is highly affected. To address this issue, each node should get medium access according to the recent information received from neighbors in the last broadcast period.

The static parameters that are used in the 802.11p standard [4] do not take into account the number of communicating nodes [16], the speed of each node [3], the adaptivity of the backoff mechanism [7], and the fluctuation in vehicle density. All of these factors affect the efficiency and sharing of the communication medium. To achieve better network performance, our proposed DCF function addresses many factors that have significant impact at the MAC layer. These are the vehicle's speed, its position, and the number of one-hop neighbors. The first two factors are related to the network topology and the communication scenario, which is a vehicular ad hoc network in our case. The last one is related to the MAC scheme, which is in our case a modified DCF function. 


\subsection{Network Topology and Configuration}

In the system model under consideration, mobile nodes communicate via a single physical channel in a pure ad hoc mode. The channel is assumed to be error free. Each node has a unique ID, based on its MAC address. Moreover, vehicles cooperate in the ad hoc mode, and each node relays packets whenever a multihop connection is established. All nodes have identical MAC functions and equal transmission capabilities. Each node sends its packets to a specific destination according to a routing protocol. Here, we focus on MAC for single-hop transmissions. When the packets are broadcasted, the message is sent to the one-hop neighbors.

\subsection{MAC Layer}

Vehicles operate at the MAC level according to the standard IEEE 802.11 DCF function. As in a WLAN, IEEE 802.11p is based on the DCF mechanism which is a CSMA/CA-based protocol. A CSMA/CA protocol basic function assumes a specific backoff mechanism. Typically, a node senses a channel and if it finds the channel busy, it backoffs for a certain time that is uniformly distributed between 0 and $C W+1$, where $C W$ is the contention window size. Otherwise, the node transmits its packets. Each node with a specific type of traffic has an initial value for $C W$ called the minimum contention window $\left(C W_{\min }\right)$. Figure 3.1 illustrates the channel status of the IEEE 802.11 DCF.

The IEEE 802.11p uses the static parameters of the IEEE 802.11e EDCA. The EDCA function provides traffic priorities as given in Table 3.2 . To find the exact values for $C W_{\min }$ and $C W_{\max }, a C W_{\min }$ and $a C W_{\max }$ should be 15 and 1023 , respectively. When evaluating 


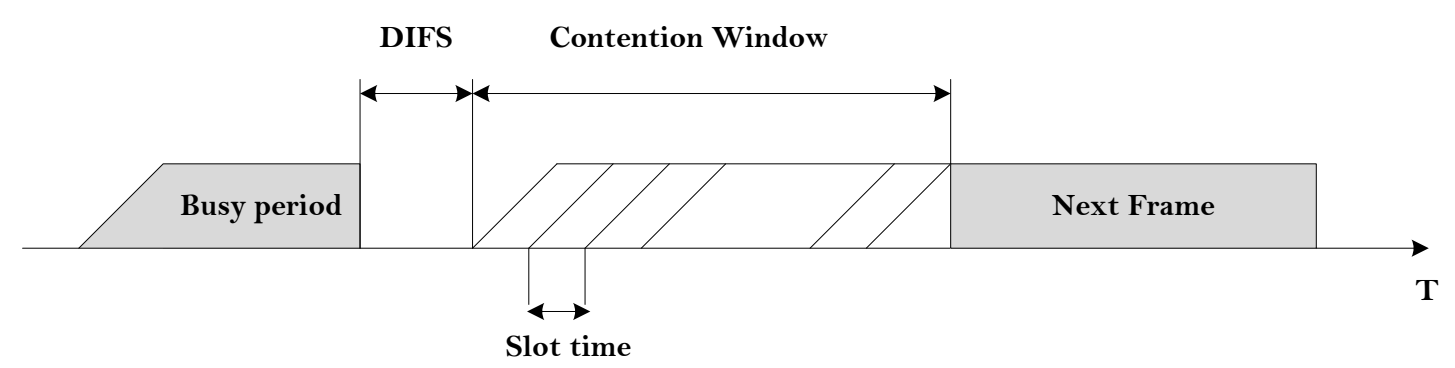

Figure 3.1: Illustration of the DCF operation.

the IEEE 802.11p, we set the static parameters of the contention windows according to the IEEE 802.11e standard.

\begin{tabular}{cccc}
\hline $\mathrm{AC}$ & $C W_{\min }$ & $C W_{\max }$ & $\mathrm{AIFSN}$ \\
\hline $\mathrm{VI}$ & $\frac{\left(a C W_{\min }+1\right)}{4}-1$ & $\frac{\left(a C W_{\min }+1\right)}{2}-1$ & 2 \\
$\mathrm{VO}$ & $\frac{\left(a C W_{\min }+1\right)}{4}-1$ & $\frac{\left(a C W_{\min }+1\right)}{2}-1$ & 3 \\
$\mathrm{BE}$ & $\frac{\left(a C W_{\min }+1\right)}{2}-1$ & $a C W_{\min }$ & 6 \\
$\mathrm{BK}$ & $a C W_{\min }$ & $a C W_{\max }$ & 9 \\
\hline
\end{tabular}

Table 3.1: Different parameters values for different traffic categories in 802.11p EDCA.

Each vehicle is equipped with a GPS receiver that can determine its position and speed. Each node maintains a list of one-hop neighbors, and periodically broadcasts a HELLO message that includes its information to the neighbors. All the neighboring nodes store the information for a certain time (e.g., 2-3 seconds [16]). If a node does not hear any information from a previous neighbor for a while, that neighbor will be removed from the neighbor list. At the end of a broadcasting period, each node calculates the average speed of itself and its one-hop neighbors, and the deviation of its speed from the average. The deviation from the average speed will be considered in the dynamic priority management 
in channel access (to be discussed in Section 3.3.2). Time is partitioned into frames of a constant length. Figure 3.2 shows the time frame for the periodic broadcasting and the IEEE $802.11 \mathrm{p}$ contention-based channel access period. At the beginning of a time frame, a cluster formation is performed. Each cluster is maintained by a clusterhead. A clusterhead broadcasts a message that assigns the mini-slots in the broadcasting period to the clustermembers. Every node that receives the clusterhead's message knows its minislot, and is synchronized with the other clustermembers. Therefore, there are no collisions during the HELLO broadcasting period.

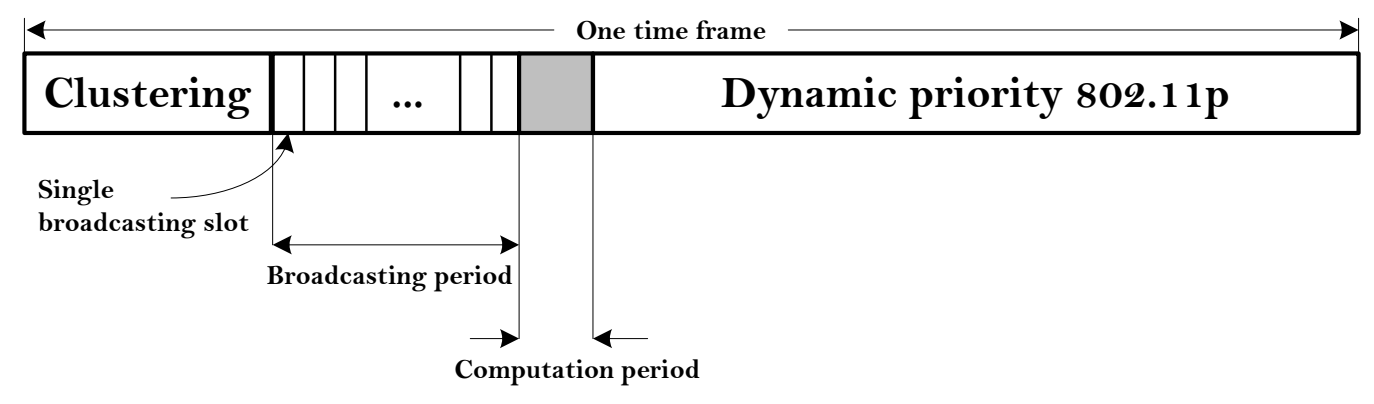

Figure 3.2: Frame structure with periodic broadcasting of vehicle information.

\subsection{MAC Adaptivity to Mobility}

Here, we present two priority channel access schemes based on vehicle mobility. Both schemes aims at optimizing the backoff mechanism in the MAC protocol by assigning dynamic contention window sizes based on node mobility parameters. The first scheme is a $p$-persistent carrier sense multiple access with collision avoidance (CSMA/CA) based backoff mechanism, while the second one is a dynamic priority management scheme based on node relative velocity. 


\subsubsection{Adaptation to the Number of Neighboring Nodes}

The IEEE 802.11 DCF function is modeled in the literature differently. Bianche proposes a complex model based on a Markove chain [47], and provides a performance analysis of the DCF in the saturated mode. However, another model for the DCF is based on the p-persistent CSMA/CA. Several researchers showe that $p$-persistent CSMA/CA provides very close approximation for the IEEE 802.11 [48] [49] [50] [51] [52].

To provide adaptivity to the number of neighboring nodes, we model the backoff procedure of the IEEE 802.11p as a p-persistent CSMA/CA. The main difference between the $p$-persistent 802.11 and the standard IEEE 802.11 p protocol is only in the selection of the backoff interval. In the standard protocol, the backoff interval is binary exponential. However, in the $p$-persistent CSMA/CA, the backoff interval is based on a geometric distribution with a specific probability of transmission, $p$. Therefore, the probability that a node stays idle when having a busy medium is $1-p$. The $p$-persistent CSMA/CA provides very close approximation to the IEEE 802.11 [48] [50] [51][52], and the memoryless backoff property makes it suitable for the purpose of analysis.

Based on the geometrically distributed backoff, the probability of having a success after $n-1$ failures of Bernoulli trials is

$$
P(X=n)=(1-p)^{n-1} p, \quad n=1,2, \ldots .
$$

Accordingly, based on [48] and [51, the expected value of the random variable $X$ can be used to determine the average contention window size $\overline{C W}$ as

$$
E[X]=\sum_{n=1}^{\infty} n p(1-p)^{n-1}=\frac{1}{p}
$$




$$
\frac{\overline{C W}+1}{2}=\frac{1}{p} .
$$

There are several important probabilities that we should consider. Consider that, at the beginning of a transmission, a node has probability $p$ to transmit. We have

$$
\begin{aligned}
P\{0 \text { transmissions }\} & =(1-p)^{M} \\
P\{\text { only one transmission }\} & =M p(1-p)^{M-1} \\
P\{\text { at least one transmission }\} & =1-(1-p)^{M}
\end{aligned}
$$

where $M$ is the number of contending nodes. Then, the probability of a successful transmission, $P_{s}$, and the probability of a collision, $P_{c}$, are given by

$$
\begin{aligned}
P_{s} & =P\{\text { Trans. nodes }=1 \mid \text { Trans. nodes } \geq 1\} \\
& =\frac{M p(1-p)^{M-1}}{1-(1-p)^{M}} \\
P_{c} & =P\{\text { Trans. nodes } \geq 2 \mid \text { Trans. nodes } \geq 1\} \\
& =\frac{1-(1-p)^{M}-M p(1-p)^{M-1}}{1-(1-p)^{M}} .
\end{aligned}
$$

In [51, a virtual transmission time $\left(V_{T}\right)$ is defined to be the time interval between two adjacent successful transmissions for all the nodes in a cluster. Figure 2.4 shows an example of the $V_{T}$. It is possible to have a number of collisions in addition to one successful transmission, in a $V_{T}$.

Let $T_{i}$ denote the idle time during which no vehicle is transmitting, $T_{s}$ the time of successful transmissions, and $T_{c}$ the total time of transmission collisions, within a virtual 


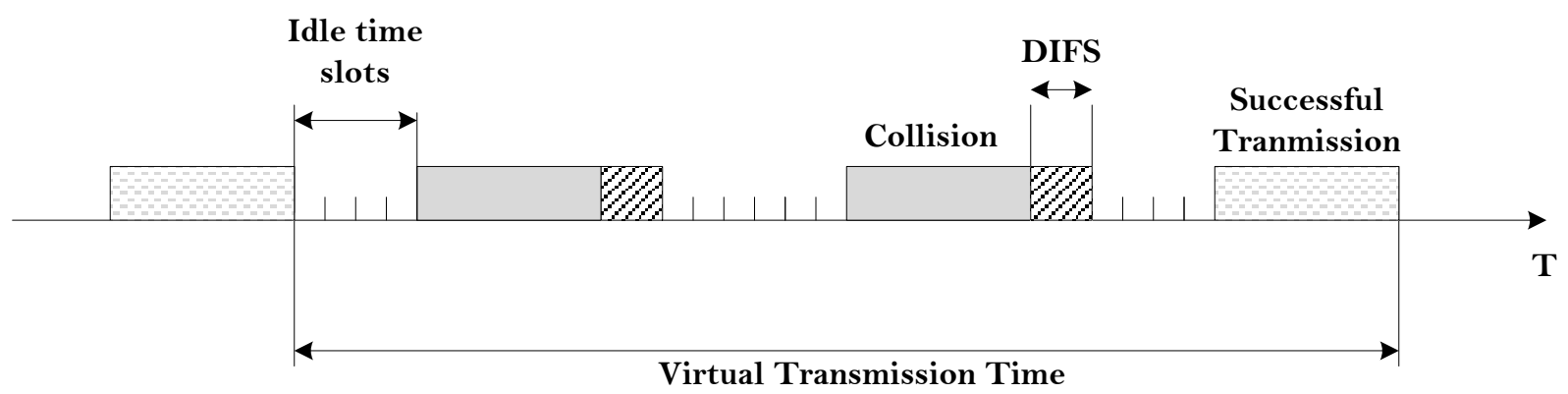

Figure 3.3: Illustration of a virtual transmission time.

transmission time. Then, we have [52]

$$
E\left[V_{T}\right]=E\left[T_{i}\right]+E\left[T_{c}\right]+E\left[T_{s}\right] .
$$

Based on the defined probability of transmissions and since the packet length, $L$, is fixed, mathematical expressions for $E\left[T_{i}\right], E\left[T_{c}\right], E\left[T_{s}\right]$ can be obtained [51][52]. For maximum system performance in terms of throughput, the value of $V_{T}$ should be minimized. Let $L$, $D$ and $\delta$ denote the length of the packet, the DIFS time, and the slot time, respectively. Then

$$
\begin{aligned}
& E\left[T_{i}\right]=\left[\frac{1-(1-p)^{M}-M p(1-p)^{M-1}}{M p(1-p)^{M-1}}\right]\left(\frac{1-p}{M p}\right) \delta \\
& E\left[T_{c}\right]=\left[\frac{1-(1-p)^{M}-M p(1-p)^{M-1}}{M p(1-p)^{M-1}}\right](L+D) \delta \\
& E\left[T_{s}\right]=(L+D) \delta
\end{aligned}
$$


and we have

$$
\begin{aligned}
E\left[V_{T}\right] & =\left[\frac{1-(1-p)^{M}-M p(1-p)^{M-1}}{M p(1-p)^{M-1}}\right]\left(\frac{1-p}{M p}\right) \delta \\
& +\left[\frac{1-(1-p)^{M}-M p(1-p)^{M-1}}{1-(1-p)^{M-1}}\right](L+D) \delta \\
& +(L+D) \delta .
\end{aligned}
$$

By using basic algebra, we have

$$
E\left[V_{T}\right]=\left[\frac{(L+D)-(L+D-1) \cdot(1-p)^{M}}{M p(1-p)^{M-1}}\right] \delta
$$

The optimal transmission probability, $p_{\text {opt }}$, which minimizes the value of $E\left[V_{T}\right]$, can be

obtained by equating the first derivative of $E\left[V_{T}\right]$ with respect to $p$ to zero. Given values of $L, D$, and $M, p_{\text {opt }}$ can be numerically computed.

In the proposed MAC protocol, the $p_{\text {opt }}$ value is used to tune the contention window size to reach the desired performance. To that end, each node that wants to transmit should already have the number of one-hop contending nodes, $M$. With $p_{\text {opt }}$, a suitable value for the minimum contention window sizes is assigned based on (3.2). A summary of symbols and variables used in the model is given in Table 3.2 .

\subsubsection{Adaptation to Vehicle Velocity}

This proposed MAC scheme uses a relation between the relative speed and the level of service priority. Basically, the deviation of the node speed from the average speed of the neighbors is proportional to the level of channel access priority. In other words, over each constant observation interval, the share of the channel time for a node with the average 


\begin{tabular}{c|l}
\hline Variable & Meaning and description of the variable \\
\hline \hline$p$ & Probability of transmission in a time slot. \\
\hline$M$ & Number of transmitting nodes. \\
\hline$V_{T}$ & Virtual Transmission time. \\
\hline$L$ & Length of the packet. \\
\hline$D$ & Length of the DIFS time. \\
\hline$p_{o p t}$ & Optimal probability of transmission. \\
\hline$E\left[T_{i}\right]$ & Average total length of idle time slots. \\
\hline$E\left[T_{c}\right]$ & Average total length of collision time slots. \\
\hline$E\left[T_{s}\right]$ & Average successful transmission length in time slots. \\
\hline$\delta$ & Slot time. \\
\hline
\end{tabular}

Table 3.2: List of notations and variables used in the model.

speed is reduced and that of a node with an extremely low or high speed is increased. In this way, we want to achieve better fairness over a number of the observation intervals, in terms of how long each node shares the medium based on the estimated time it spends in the active transmission range.

For a cluster of $M$ nodes contending for the channel, the share of node $i$ accessing the channel is proportional to $\frac{p_{i}}{\sum_{j=1}^{M} p_{j}}$, where $p_{i}$ is the transmission probability of node $i$.

One way to relate the channel access time to the node velocity is to adjust the contention window size to provide service priority. For a transmitting node, $i$, with a velocity, $V_{i}$, the deviation from the average speed, $d$, is given by

$$
d=\left|V_{i}-\bar{V}\right|
$$

where $\bar{V}$ is the average speed of the one-hop neighbors in the cluster.

For simplicity in implementation, vehicles are categorized into different classes based on their speed deviations from the average speed, as given in Table 3.3.2. Accordingly, 
each vehicle adjusts the values of the minimum and maximum contention window sizes, $C W_{\min }$ and $C W_{\max }$, respectively.

\begin{tabular}{ccc}
\hline$d$ & Priority & Access Class \\
\hline Small & low & 3 \\
Medium & medium & 2 \\
Large & high & 1 \\
\hline
\end{tabular}

Table 3.3: Dynamic service priority assignment based on relative speed.

\subsection{Summary}

In this chapter, we describe the system model and the proposed priority channel access schemes. The network structure and the MAC layer are illustrated. A detailed description of the DCF function is given. Moreover, the neighbor discovery procedure through the periodic broadcasting of the HELLO message. Finally, the $p$-persistent MAC model is illustrated followed by the velocity adaptive scheme. 


\section{Chapter 4}

\section{Performance Evaluation}

\subsection{Vehicular Mobility Model}

Vehicles are assumed to move in a straight segment of highway road according to the freeway mobility model [53]. Initially, vehicles positions are uniformly distributed. Then, vehicles move according to a predetermined range of speeds and acceleration. When a vehicle leaves the highway, it re-enters from the start point. In the freeway mobility model, there are three main conditions. A node does not change lane, its speed is temporally dependent on its previous one, and a safety distance between two consecutive nodes is used to keep the following node speed lower than the speed of the preceding one. Figure 4.2 shows a sample of a 3-lane highway with vehicles moving according to the freeway mobility model. 


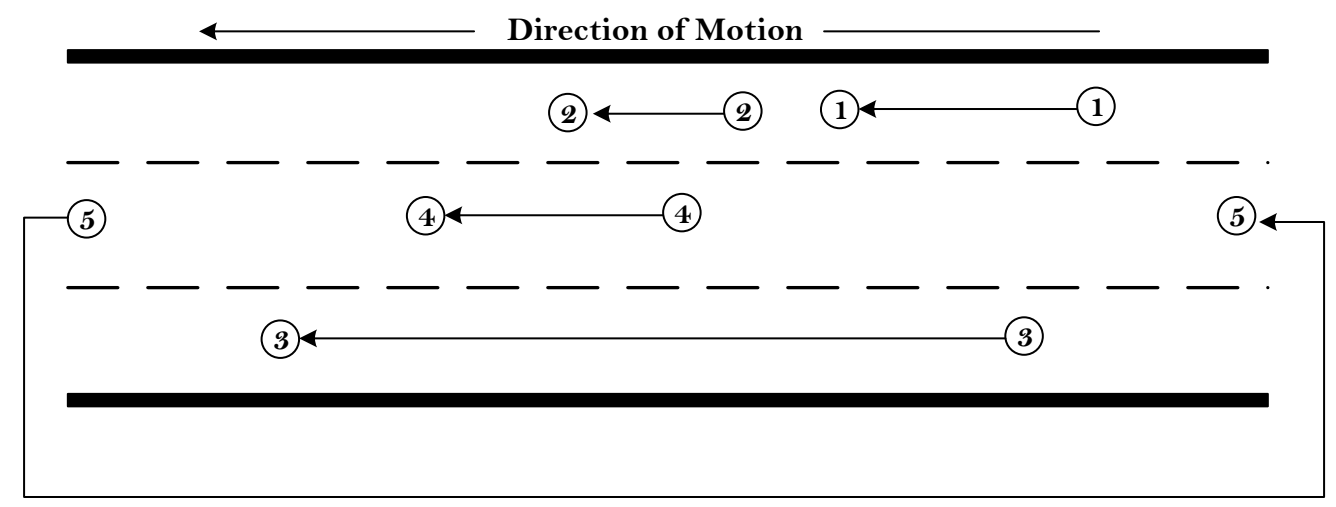

Figure 4.1: Vehicles mobility according to the freeway model.

\subsection{Simulation Parameters}

To evaluate the impact of mobility on the standard IEEE 802.11p and the proposed dynamic priority management schemes, simulations are performed using Network Simulator (NS2) [54], version 2.31. The simulations are carried out for a 3-lane highway with a length of $5 \mathrm{~km}$ and a width of $10 \mathrm{~m}$ per lane as in Figure 4.2 . Vehicle velocity varies from 60 $120 \mathrm{~km} / \mathrm{h}$. All vehicles have the same 802.11p MAC parameters. Vehicles move according to the freeway mobility model as described in [53].

In all the simulations, the system time is set to $100 \mathrm{~s}$, and the transmission range of each vehicle is $250 \mathrm{~m}$. Vehicles communicate in a V2V mode. Each vehicle either transmits packets at a constant rate, or generates packets according to a Poisson random process with mean arrival rate $\lambda=3.125$ packets per second. In the constant rate packet arrival, each node always has packets to transmit. The packet size is constant. Channel reuse is permitted in different node clusters. The number of nodes contending for the channel in the simulations varies from 20 to 200. For each number of nodes, we run four 
simulation scenarios and obtain the average of the four results. We set the parameters of the IEEE $802.11 \mathrm{p}$ at the MAC layer with time slot of $\delta=13 \mu \mathrm{s}$, and SIFS time of $32 \mu \mathrm{s}$.

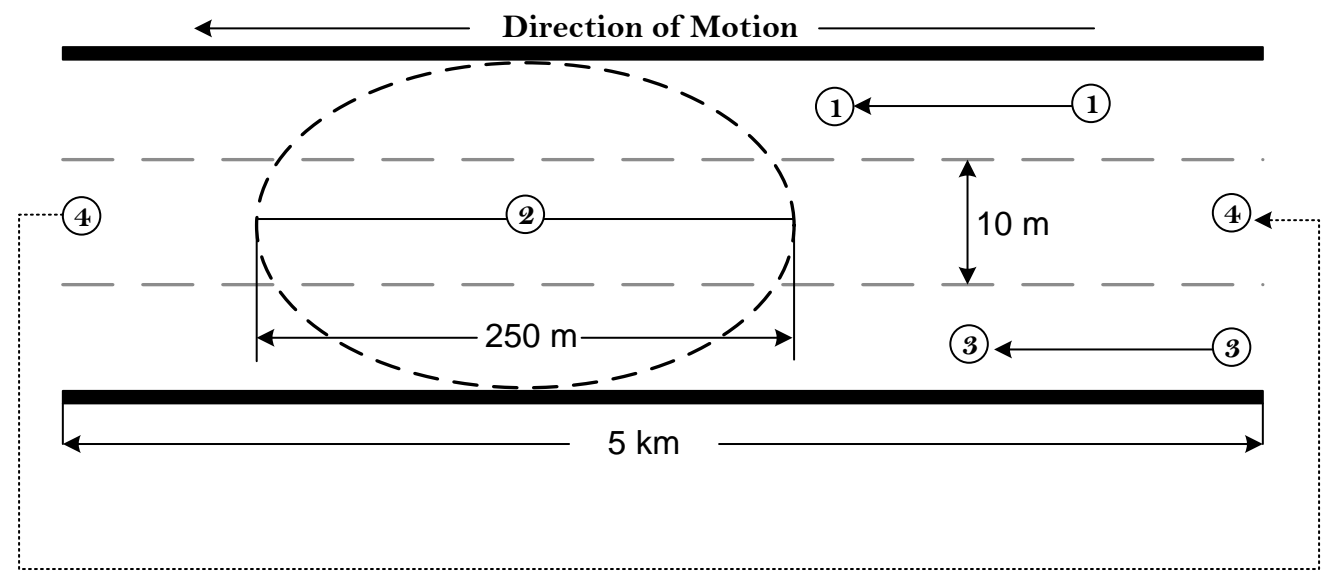

Figure 4.2: The traffic mobility scenario used in the simulation.

Consider two different communication scenarios in the evaluation. In the first one, each node broadcasts packets to its neighbors. In the second scenario, each node unicasts packets to a destination, which may not be a neighbor. We use the Ad hoc On-Demand Distance Vector (AODV) routing protocol in the unicast scenario. The packet size is fixed for each scenario. Five minimal contention window sizes are used: $C W_{\min }=3, C W_{\min }=7$, $C W_{\min }=15, C W_{\min }$ computed using the $p$-persistent scheme, and $C W_{\min }=7$ computed using the velocity adaptive scheme. Three maximal contention windows sizes are used: $C W_{\max }=7, C W_{\max }=255$, and $C W_{\max }=1023$. The rest of the parameters for are given in Table 4.1, 


\begin{tabular}{lcc}
\hline & Broadcast scenario & Unicast scenario \\
\hline Packet size & 500 Bytes & 1024 Bytes \\
Broadcast interval & $500 \mathrm{~ms}$ & $500 \mathrm{~ms}$ \\
Routing & No routing & AODV \\
Traffic Rate & $1.2 \mathrm{Mbps}$ & Poisson, $\lambda=3.125$ packets $/ \mathrm{s}$ \\
\hline
\end{tabular}

Table 4.1: Different parameters for the simulation.

\subsection{Mobility and Performance Metrics}

Unlike previous works in the literature, we use different performance metrics to evaluate the mobility impact and the network performance. The purpose of the defined metrics is twofold. First, identifying the mobility impact on the IEEE 802.11p MAC performance. For that, we investigate the effective mobility factors. Figure 4.3 shows the factors that affect the IEEE 802.11p performance in VANETs. The second purpose is to measure the system performance at the MAC layer. The performance metrics are chosen as follows.

In order to study the mobility impact and network connectivity, we use the communication duration as a performance metric. We study the communication duration per node according to its relative speed and distance between communicating vehicles. Furthermore, we study the distribution of the communication duration of a link. For two vehicles $i$ and $j$, the link distance $l$ and relative speed $v_{r}$ are defined as

$$
\begin{aligned}
v_{r} & =\left|v_{i}-v_{j}\right| \\
l & =\left|x_{i}-x_{j}\right|
\end{aligned}
$$

where $v_{i}$ and $v_{j}$ are the velocities of vehicles $i$ and $j$, respectively, and $x_{i}$ and $x_{j}$ are the x-axis positions of vehicles $i$ and $j$, respectively. 


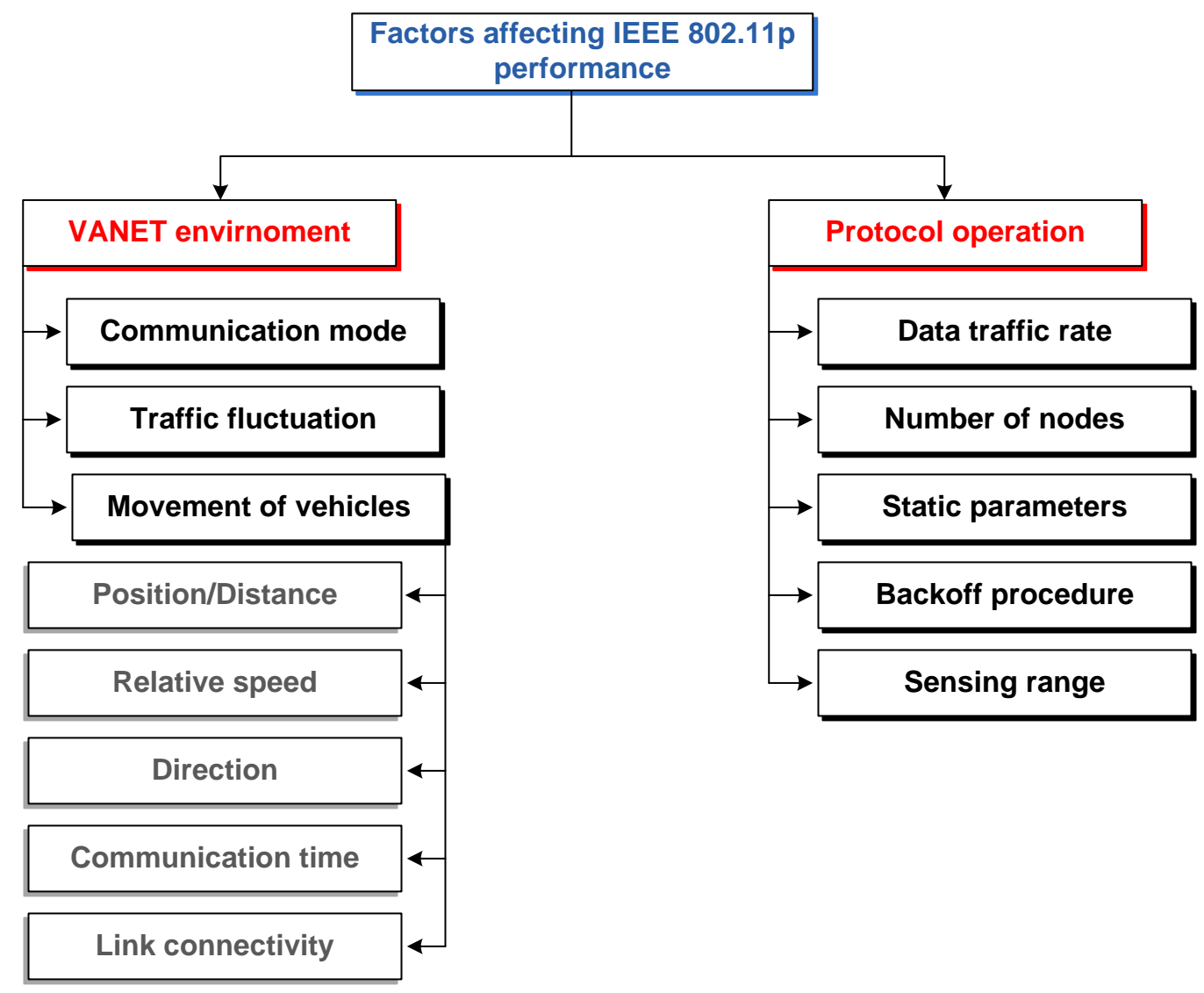

Figure 4.3: Factors affecting the IEEE 802.11p performance. 
The PDR, system throughput, average number of retransmissions per packet, average delay, and Jain fairness index [55] are used to measure the network performance at the MAC level. System throughput is the total number of bits successfully transmitted over the simulation time. These measurements indicate the efficiency of the system by showing the ratio of dropped packets, the level of contention for channel access, and the level of fairness in channel access.

\subsection{Evaluation of the Mobility Impact}

\subsubsection{Broadcast Scenario}

In the broadcast scenario, we first evaluate the number of potential communication neighbors within the transmission range as shown in Figure 4.4. It can be seen that the number of neighbors varies and does not follow a specific pattern. However, it is obvious that the distribution shifts to the right when the number of nodes increases. This indicates that, when the number of nodes increases, the connectivity of the network links increases. The number of communication neighbors is an important mobility factor, and their distribution

can be used to adapt the MAC protocol to the fluctuation in density. Figure 4.5 shows the average number of communication neighbors for different node densities.

Moreover, we study the communication duration of network links. The cumulative distribution function (CDF) shows that for more than 50-60\% of all occurrences, the potential communication time is less than $1 \mathrm{~s}$ as in Figure 4.6. This indicates that the communication time is very limited due to differences in node velocity.

For the effect of the relative speed between the vehicles on the channel access time, 


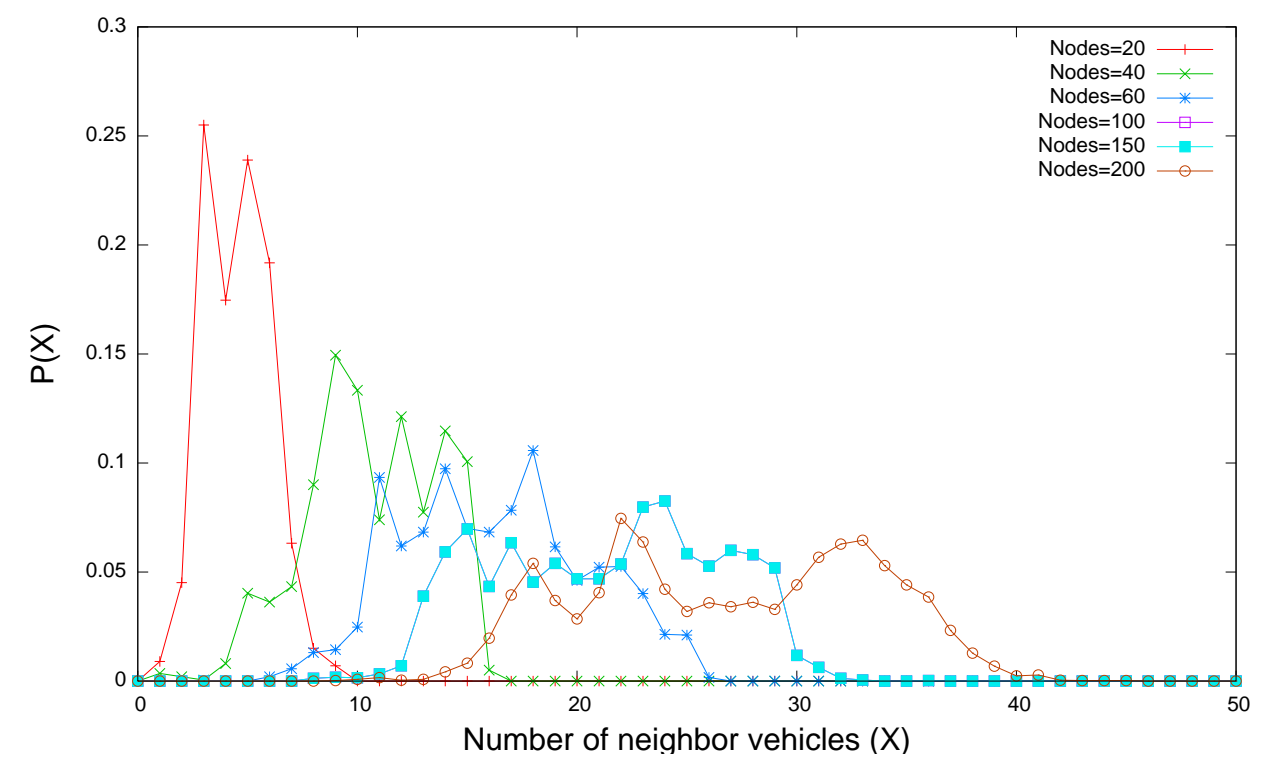

Figure 4.4: Probability mass function of the number of neighbors.

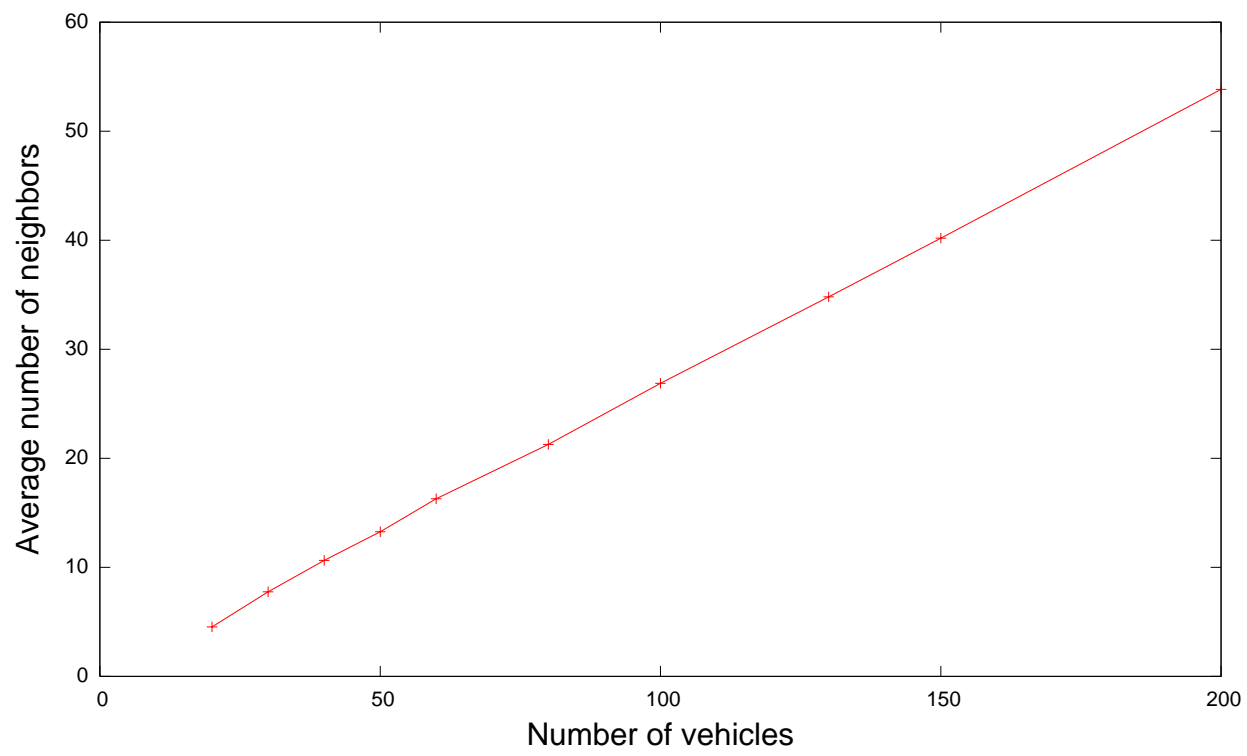

Figure 4.5: Average number of communication neighbors.

Figures 4.7(a) and 4.7(b) shows the accumulated fraction of channel access time for different node densities and different relative speeds. 


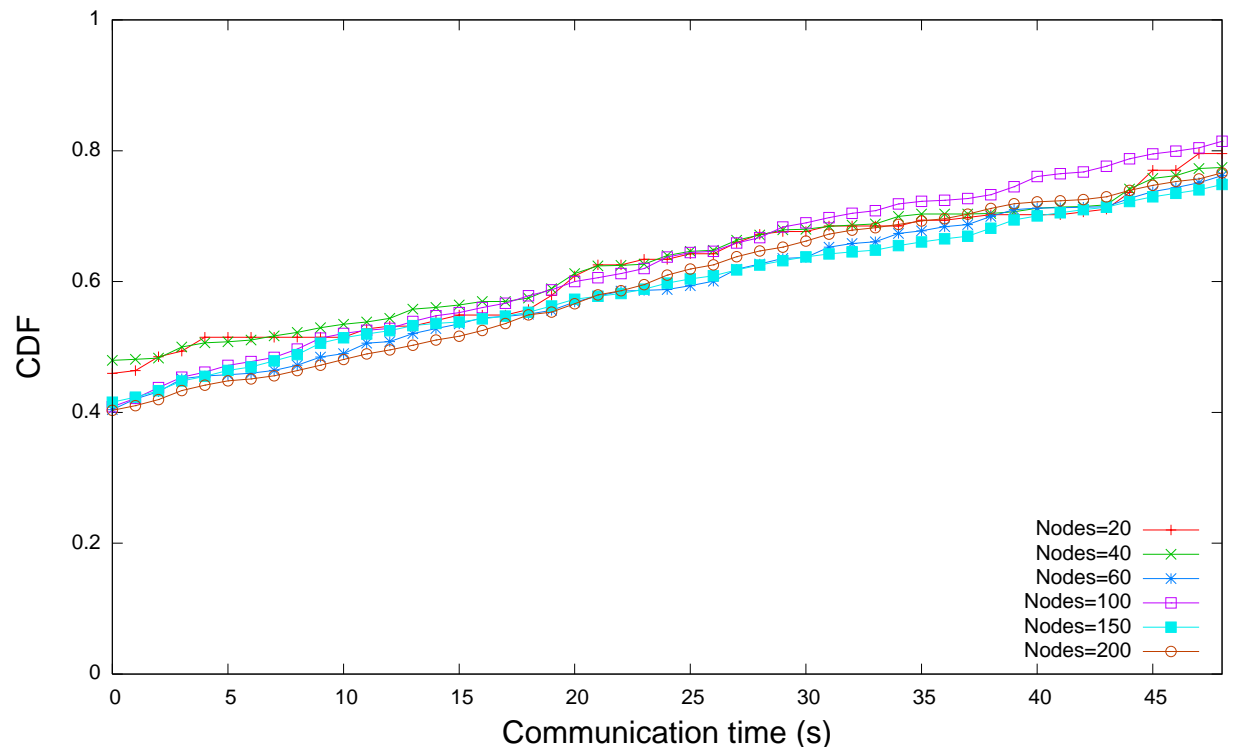

Figure 4.6: CDF of the communication duration of a link.

For a low node density, Figure 4.7(a) shows that most of the channel access time is allocated for the nodes with relative speeds less than $1 \mathrm{~m} / \mathrm{s}$. However, the rest of the results oscillates up and down but with smaller portion of channel time. This fluctuation is caused by having different number of neighbors and, accordingly, different probability of channel access, which makes the network connectivity highly unpredictable. This fluctuation almost disappears in a higher node density as in Figure 4.7(b). Figure 4.7(b) shows that most of the channel time is allocated for the nodes with relative speed less than $1 \mathrm{~m} / \mathrm{s}$, and then the access time decreases with very small fluctuations until it reaches zero with a relative speed higher than $17 \mathrm{~m} / \mathrm{s}$. Therefore, the channel access time is unfairly divided between the contending nodes according to their relative speed.

In contrast to the impact of the relative speed on the medium access, the distance between a transmitter and a receiver does not have such a huge impact as shown in Figure 4.8. Once a node is in the transmission range of the sender/receiver, it is given a channel 


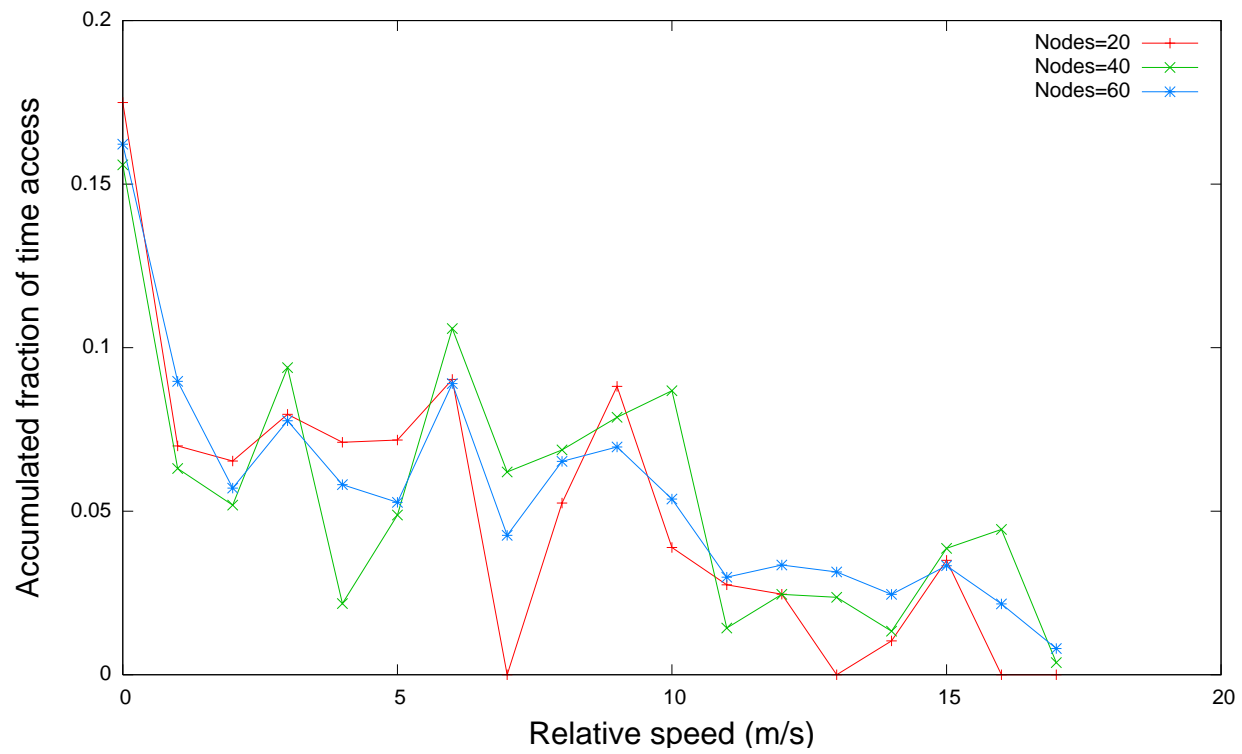

(a) Low node densities.

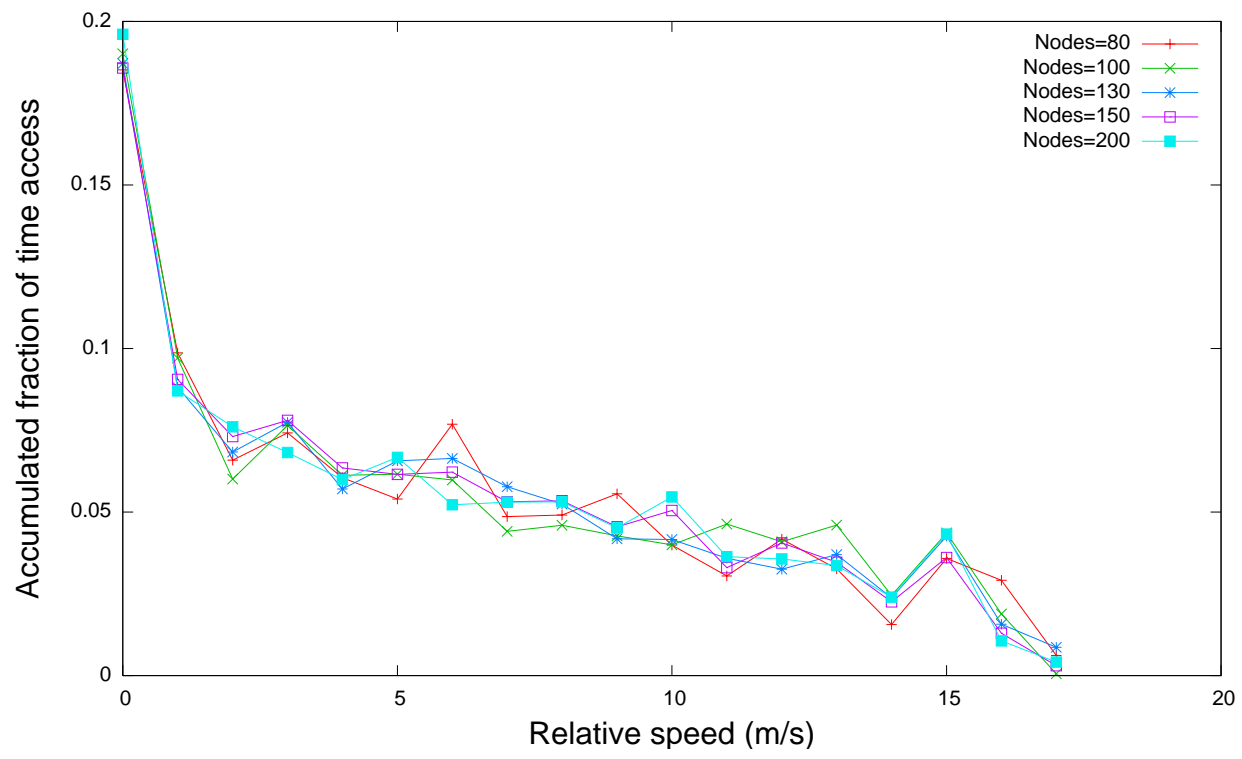

(b) High node densities.

Figure 4.7: Accumulated fraction of time access versus relative speed. 
time that depends on the other mobility factors. Figure 4.8 shows, the channel access time is spreaded over the link distance with a maximum value of $15.5 \mathrm{~ms}$.

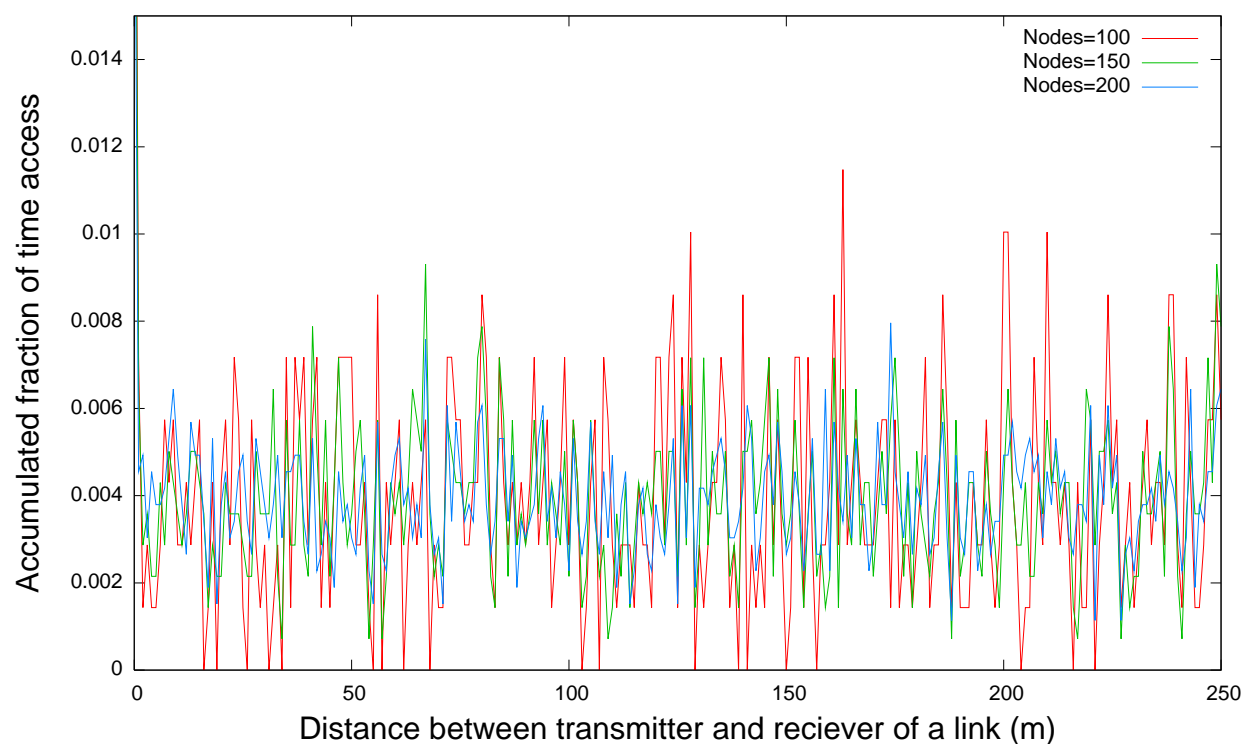

Figure 4.8: Accumulated fraction of time access versus distance.

\subsubsection{Unicast Scenario}

In the unicast scenario, each vehicle sends its packets at the routing level to a destination that may not be a neighbor. Each source and destination pair is selected randomly. The number of communication neighbors is the same as in Figure 4.4 since we are using the same mobility scenario. However, the effect of the other mobility factors on the MAC performance differs as follows. First, we evaluate the CDF of the communication duration for different node densities. Figure 4.9(a) shows the results similar to that in the broadcast scenario. It shows that, among 40-60\% of all occurrences, vehicles have less than $1 \mathrm{~s}$ to communicate. Moreover, vehicles with a relative speed less than $1 \mathrm{~m} / \mathrm{s}$ get the most of the 


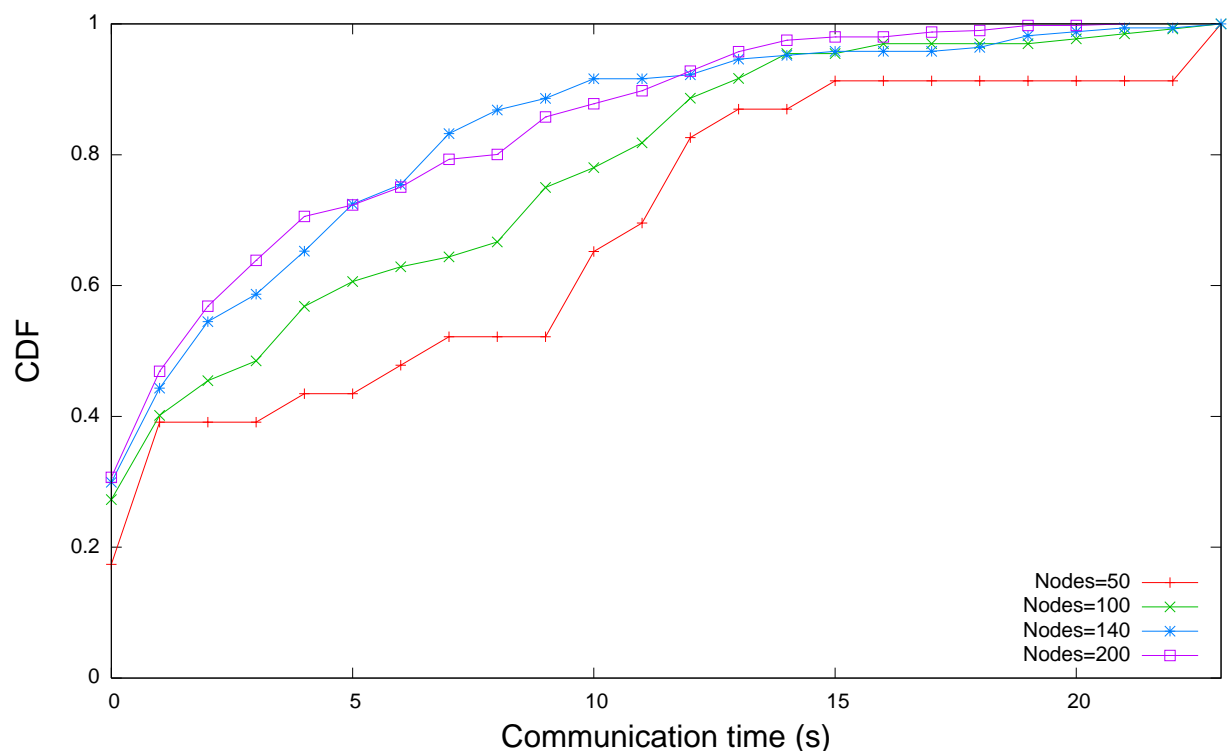

(a) CDF of the communication duration of a link.

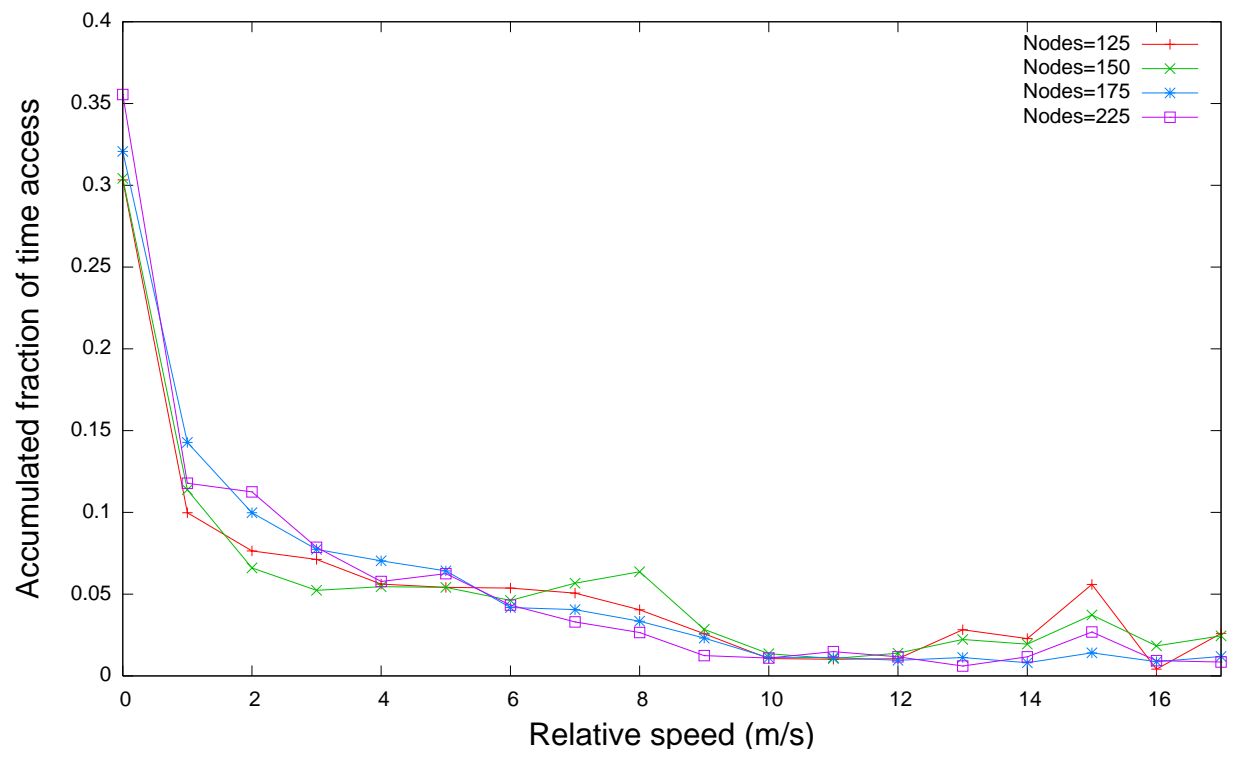

(b) Accumulated fraction of time access versus relative speed.

Figure 4.9: Mobility impact at the MAC layer in the unicast scenario.

channel time as Figure 4.9(b) shows. Unlike the broadcast scenario, the channel access time as a function of relative speed shows a similar behavior disregarding the node density, 
as Figure 4.9(b) shows. Similar to the broadcast scenario, Figure 4.10 shows that node position does not have a great impact on the medium access. The channel access time is spreaded over the link distance. However, the channel access time tends to a fraction of time less than 0.004 at link distance of $50 \mathrm{~m}$ or lower, and forms a bell shape at a link distance of 100 to $150 \mathrm{~m}$.

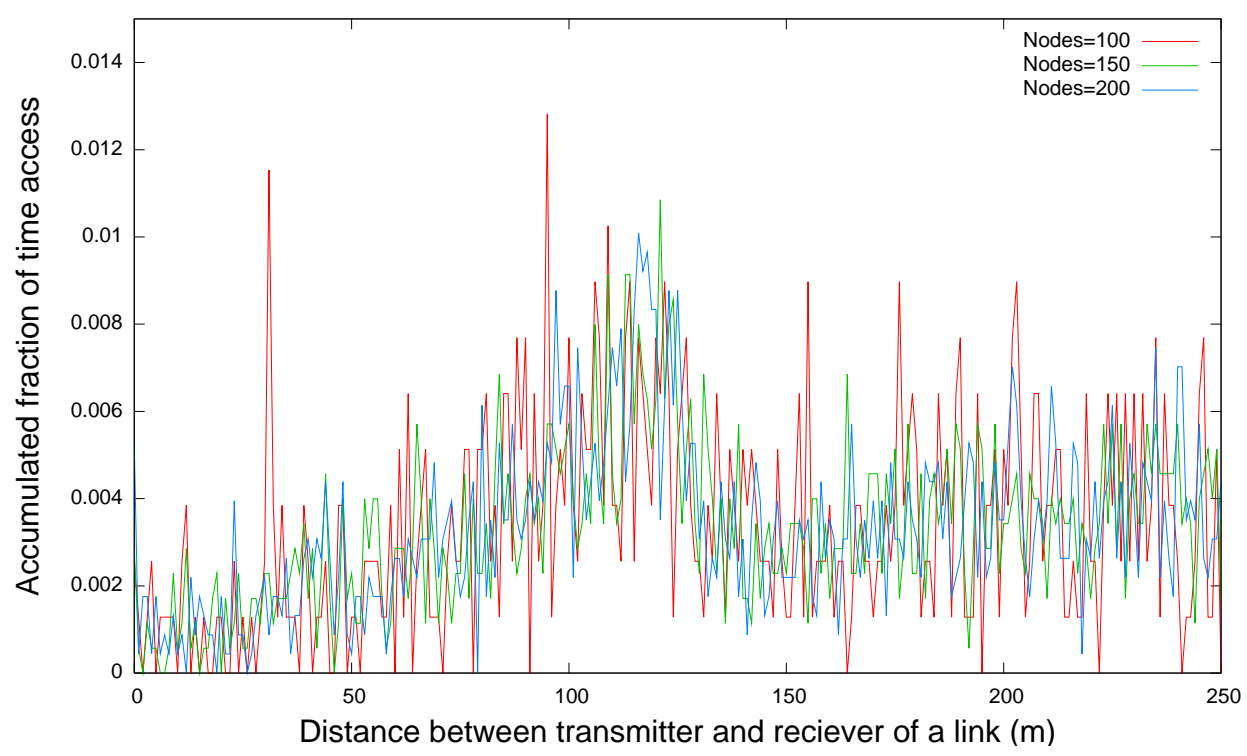

Figure 4.10: Accumulated fraction of access time versus distance.

\subsubsection{Evaluation of the IEEE 802.11p Performance}

In the previous subsections, we provide performance evaluation of the IEEE 802.11p MAC from the perspective of mobility impact. In this subsection, a different performance metric is used to evaluate the performance of the V2V communications. From now on, each node sends its packets to a destination in unicast mode. First we measure the PDR for different access categories and with packet arrival rate $R=9.9$ packets per second as Figure 4.11(a) 
shows. The PDR starts at a high value. However, when the number of nodes increases, the PDR drops to below $75 \%$ due to the frequent network partitions and lack of adaptivity to mobility factors. Similarly, the average number of retransmissions per packet is low with a low number of nodes, but increases drastically with number of nodes of 50 or more as in Figure 4.12(a), A large number of retransmissions indicates that the level of contention on channel access is severe.

Figure 4.13(a) shows that the throughput of the system increases as the number of nodes increases. This is a normal behavior of the network since more transmitting nodes lead to more delivered packets. Figure 4.14(a) shows that the delay of the successfully delivered packets at the MAC level is always under 0.4 ms. Interestingly, Jain Fairness Index indicates poor fairness at a low number of nodes, as shown in Figure 4.15(a). This is due to the frequent fragmentation of the network which causes some nodes to have less connectivity than the others. At a higher node number, the fairness index increases.

For the Poisson data traffic with mean $\lambda=3.125$ packets per second, the IEEE $802.11 \mathrm{p}$ reveals similar behavior. Figure 4.11(b) shows that the PDR drops when the number of nodes increases. Figure 4.12(b) shows that the average number of retransmission is low at a low node number and increases to around 7.5 retransmissions at a node number of 250. However, it can bee seen that the number of retransmissions is lower than that in the constant packet rate scenario as Figure 4.12(a) shows. This is due to the fact that the Poisson data arrival rate chosen is lower than that in the constant rate scenario. System throughput increases when the number of nodes increases as in Figure 4.13(b), The delay is always less than $0.45 \mathrm{~ms}$ as Figure 4.14(b) shows. In terms of fairness, Jain fairness index indicates better fairness than that in the constant packet arrival rate scenario, but with similar behavior as in Figure 4.15(b) shows. In other words, the IEEE 802.11p standard 


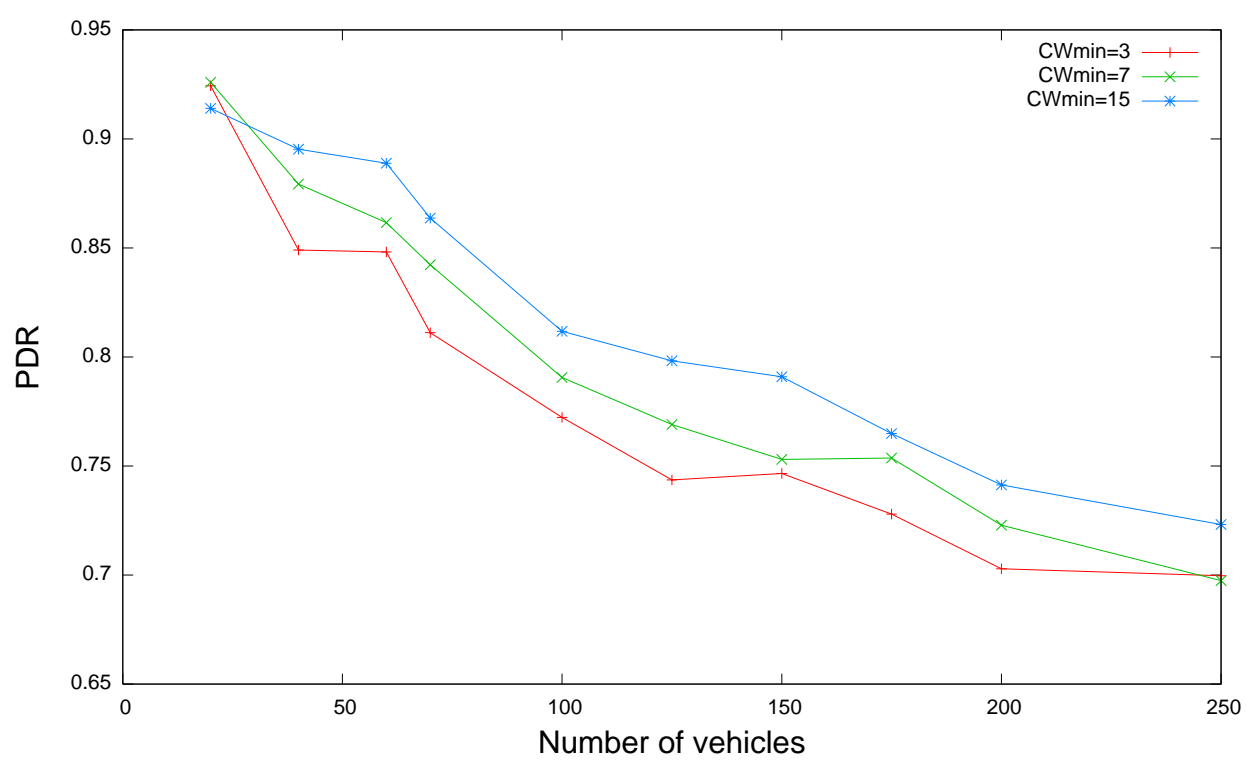

(a) Constant packet arrival rate

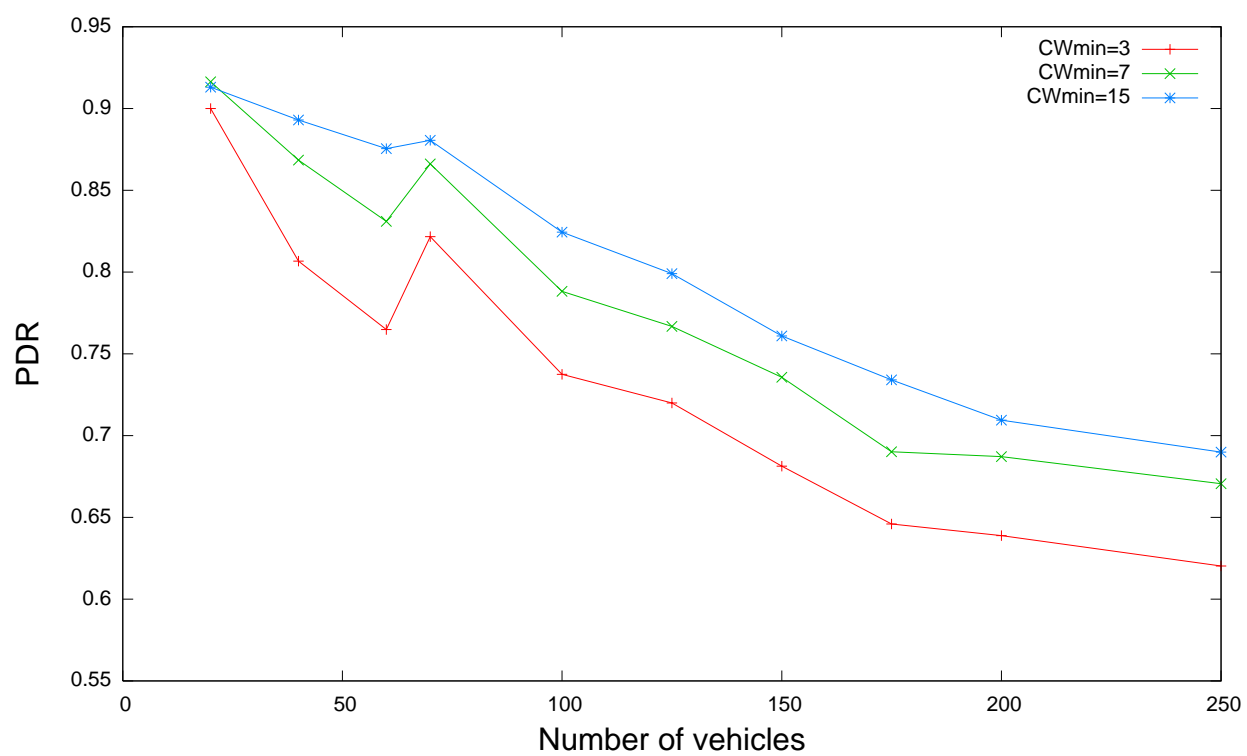

(b) Poisson packet arrival rate

Figure 4.11: PDR of the IEEE 802.11p.

shows better fairness when the packet arrival rate is Poisson. 


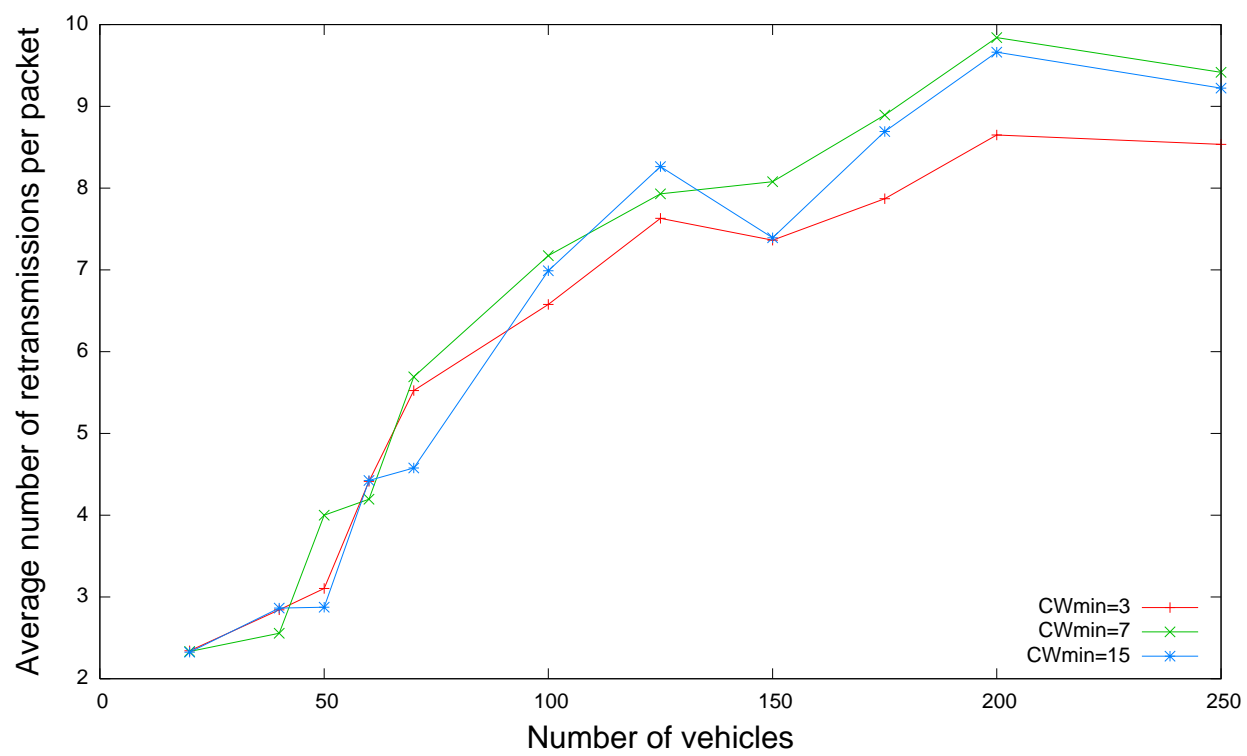

(a) Constant packet arrival rate

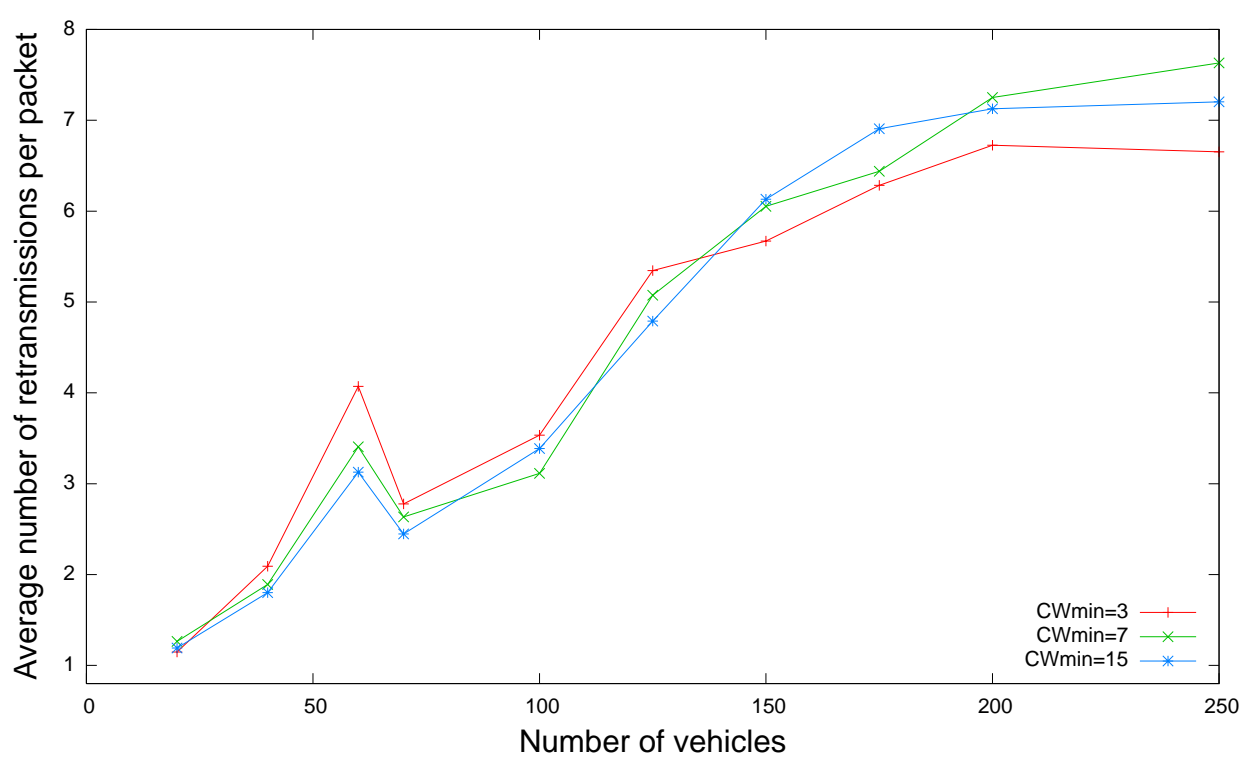

(b) Poisson packet arrival rate

Figure 4.12: Average number of retransmissions of the IEEE 802.11p. 


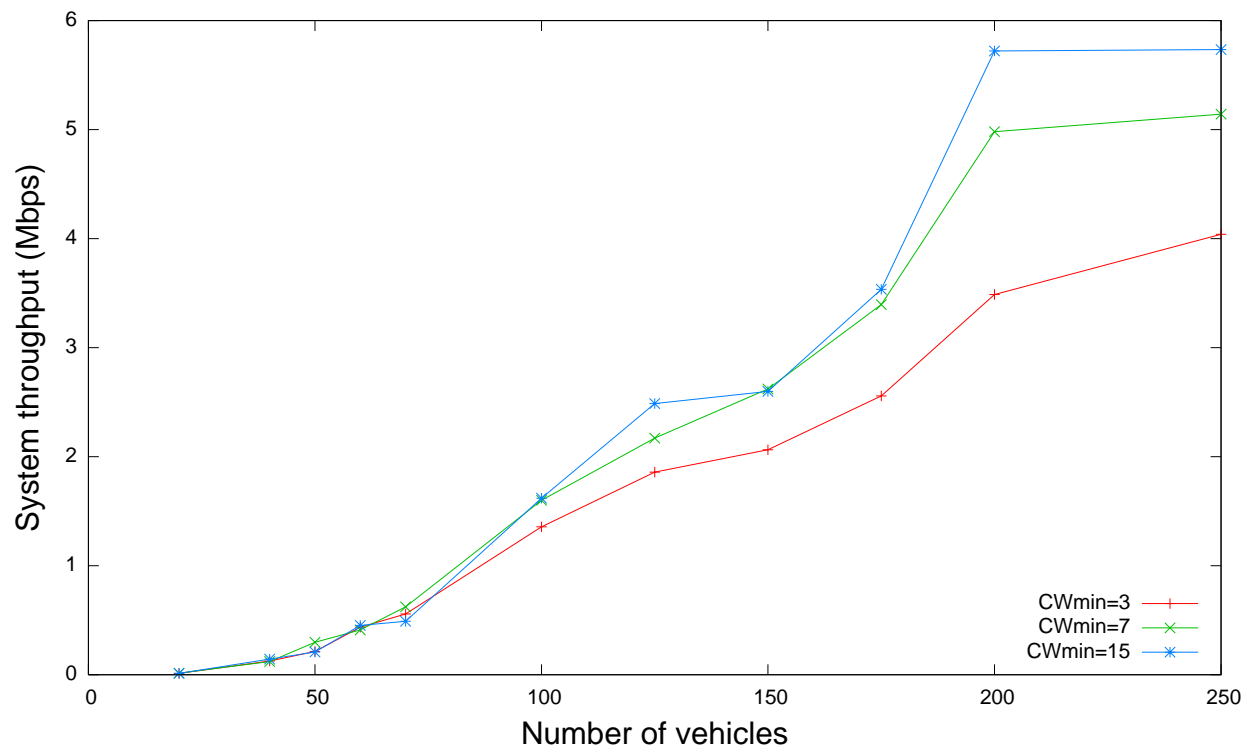

(a) Constant packet arrival rate

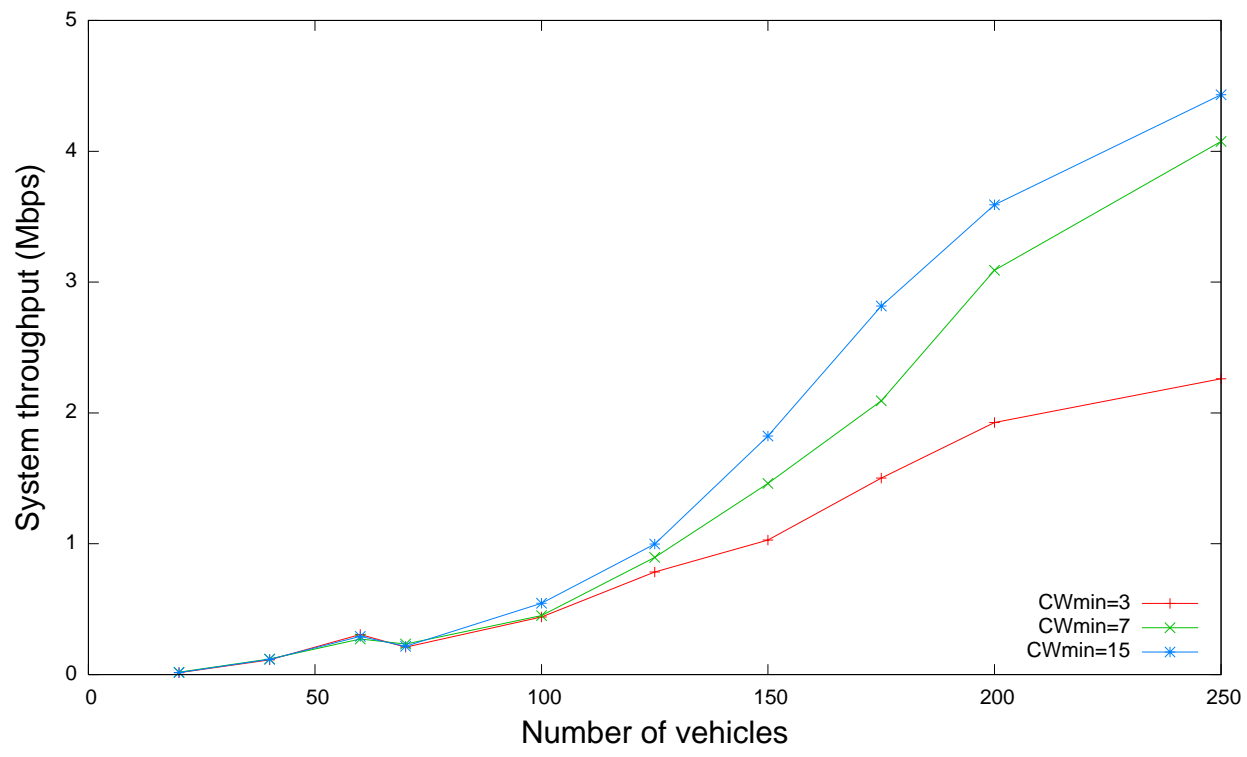

(b) Poisson packet arrival rate

Figure 4.13: Throughput of the IEEE 802.11p. 


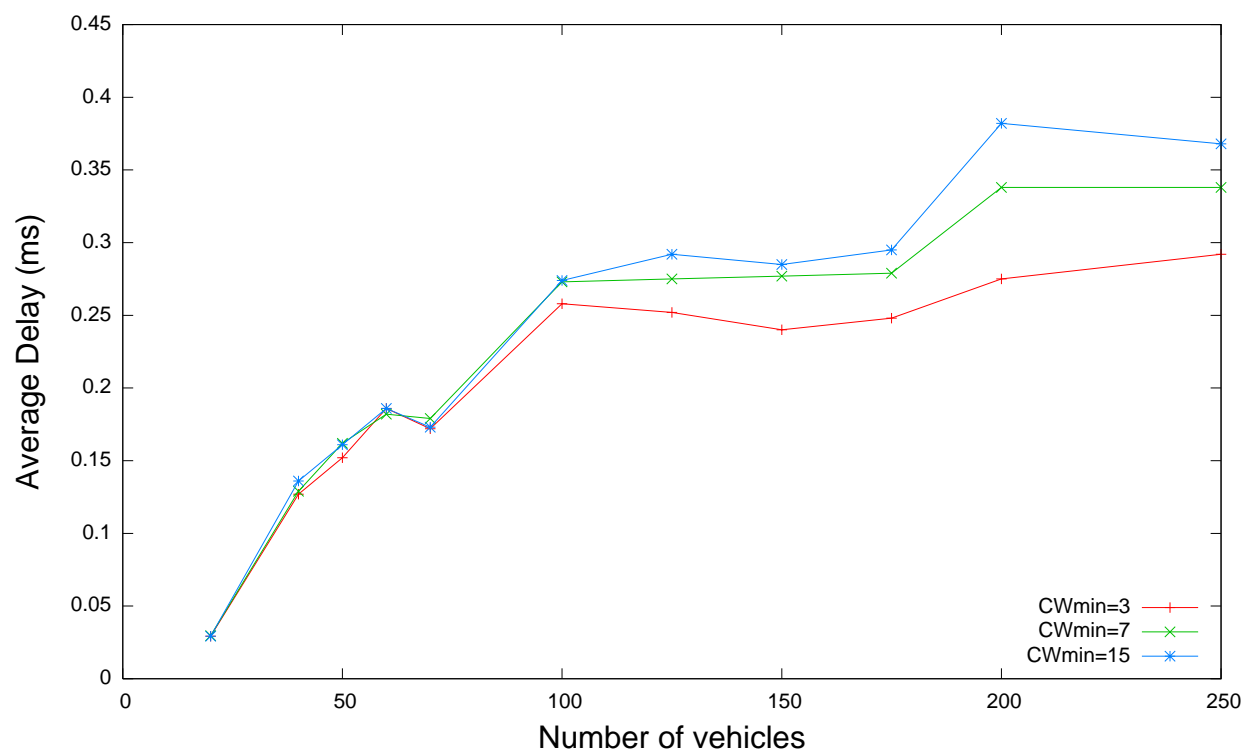

(a) Constant packet arrival rate

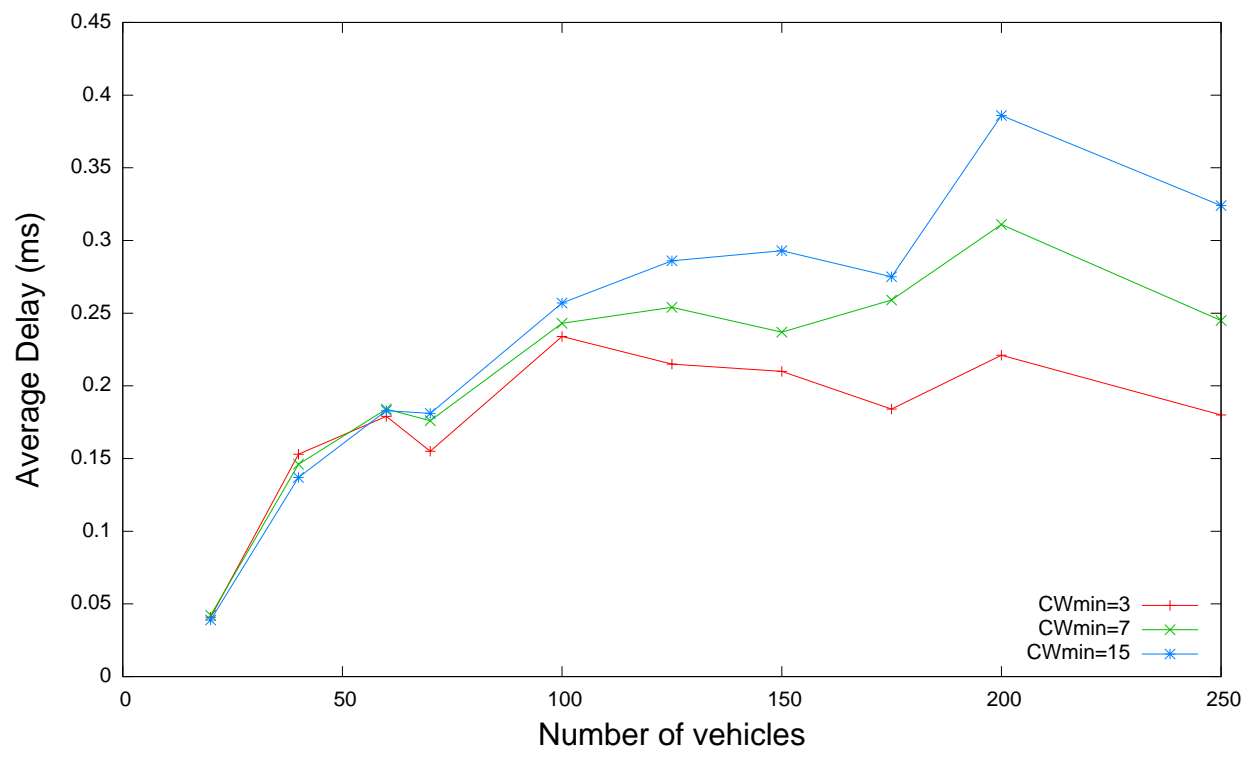

(b) Poisson packet arrival rate

Figure 4.14: Transmission delay of the IEEE 802.11p. 


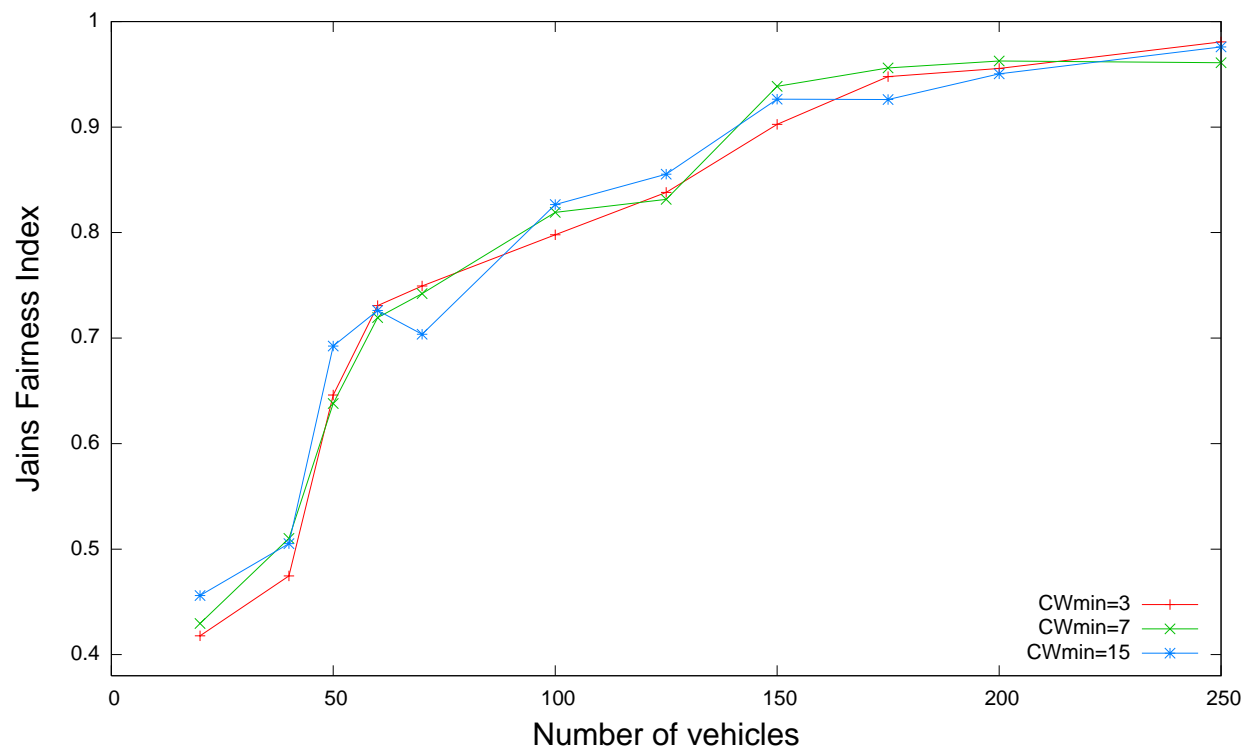

(a) Constant packet arrival rate

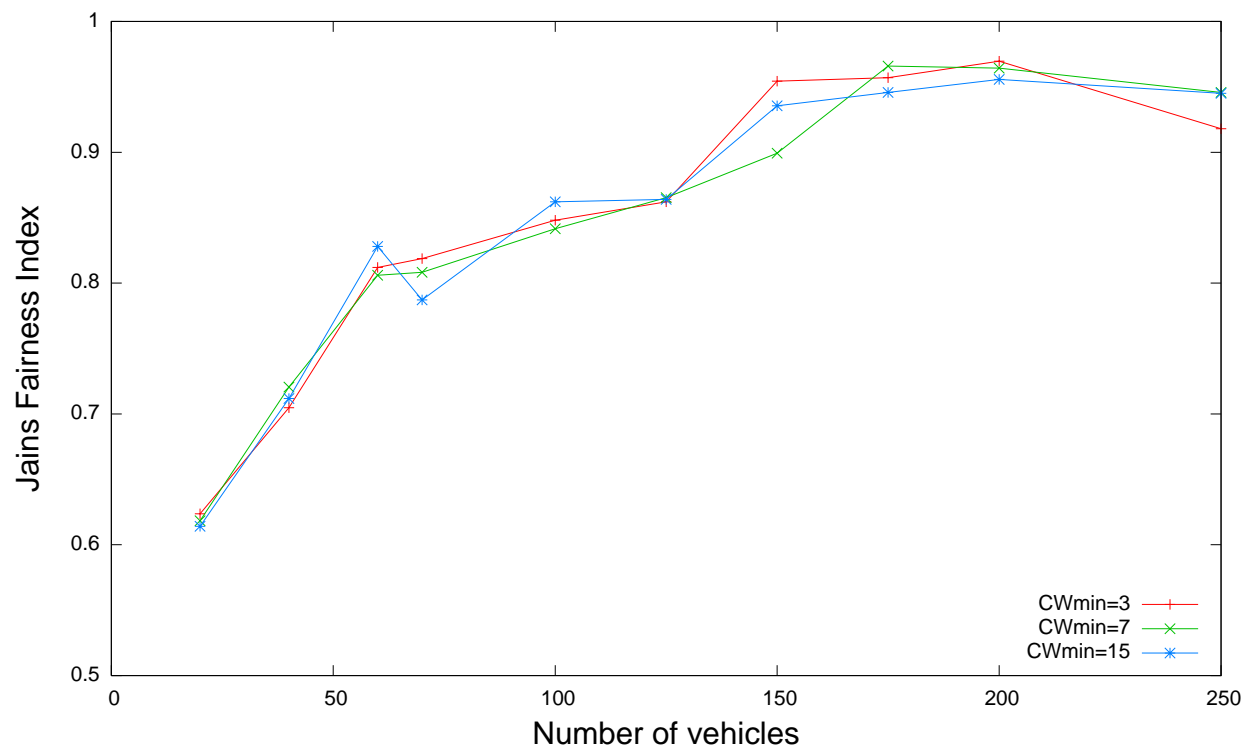

(b) Poisson packet arrival rate

Figure 4.15: Jain Fairness Index of the IEEE 802.11p. 


\subsection{Evaluation of the Adaptive Schemes}

The simulation parameters are the same as in Section 4.2. Vehicles communicate in a V2V mode. Each vehicle sends its packets at constant rate $R=9.9$ packets per second, or according to a Poisson process with arrival rate $\lambda=3.125$ packets per second. Moreover, each node broadcasts its HELLO message every $500 \mathrm{~ms}$. We run the simulation for the IEEE 802.11p standard and compare it with each proposed scheme. For the velocity adaptive MAC, the values for the speed deviation from average speed and the respective channel access priorities are given in Table 4.2. Accordingly, each vehicle adjusts the values of $C W_{\min }$ and $C W_{\max }$. The dynamic priority channel access should give nodes with high mobility the privilege of transmission over the nodes with a similar mobility behavior.

\begin{tabular}{cccc}
\hline Speed deviation $(\mathrm{m} / \mathrm{s})$ & Priority & $C W_{\min }$ & $C W_{\min }$ \\
\hline $0-3$ & low & 15 & 1023 \\
$3-10$ & medium & 7 & 255 \\
$10-17$ & high & 3 & 7 \\
\hline
\end{tabular}

Table 4.2: Dynamic assignment of parameters according to relative speed.

For the constant packet arrival rate, Figure 4.16(a) shows that the p-persistent MAC protocol results in a higher PDR than the IEEE 802.11p standard. Allowing different service priorities based on the neighbor number shows obvious alleviation of the number of dropped packets. The average number of retransmissions in the proposed $p$-persistent MAC protocol is lower than that in the IEEE 802.11p, as in Figure 4.17(a), in almost all the numbers of nodes. Therefore, the severity of contention on channel access is obviously reduced.

The $p$-persistent scheme reveals close performance to that of the IEEE 802.11p in terms 
of throughput with number of node less than 150 as shown in Figure 4.18(a). However, at node density of 50 or higher, the p-persistent scheme outperforms the IEEE 802.11p. Average transmission delay is significantly improved with a maximum value of $0.15 \mathrm{~ms}$ as Figure $4.19(\mathrm{a})$.

Even though the velocity adaptive MAC does not use the exact relative velocity between vehicles, simulation results show improvement in the performance as can be seen in Figures 4.16(a), 4.17(a), 4.18(a), and 4.19(a). The main improvement is that the protocol reduces the number of high priority packets that cause packets collisions [6] and alleviates the network performance degradation as shown in Figure 4.11(a) in Section 4.4.3. Moreover, the average number of retransmissions per packet is lower than that of the IEEE 802.11p. The velocity adaptive scheme outperforms both the $p$-persistent scheme and the IEEE 802.11p standard in terms of system throughput at a node density of 150 or higher. The average transmission delay is improved as Figure 4.20(a) shows.

Overall, both newly proposed schemes outperform the IEEE 802.11p. However, they tend to be competitive and each protocol provides better performance in certain scenarios. This is due to the fact that each MAC protocol is adaptive to one of the significant mobility parameters. Interestingly, all three MAC protocols exhibit a similar behavior at a very small node density. For the delivery ratio, both schemes outperform the IEEE 802.11p. However, there is much uncertainty of which one performs better than the other except at a high node density as in Figure 4.16(a), At a high node density, the $p$-persistent scheme performs better than the velocity adaptive scheme. This is due to the large backoff times and the fact that, the $p$-persistent scheme operate as a function of the number of onehop neighbors. For the number of retransmissions and system throughput, the velocity adaptive scheme outperforms the $p$-persistent scheme at a node density of 80 or higher as 


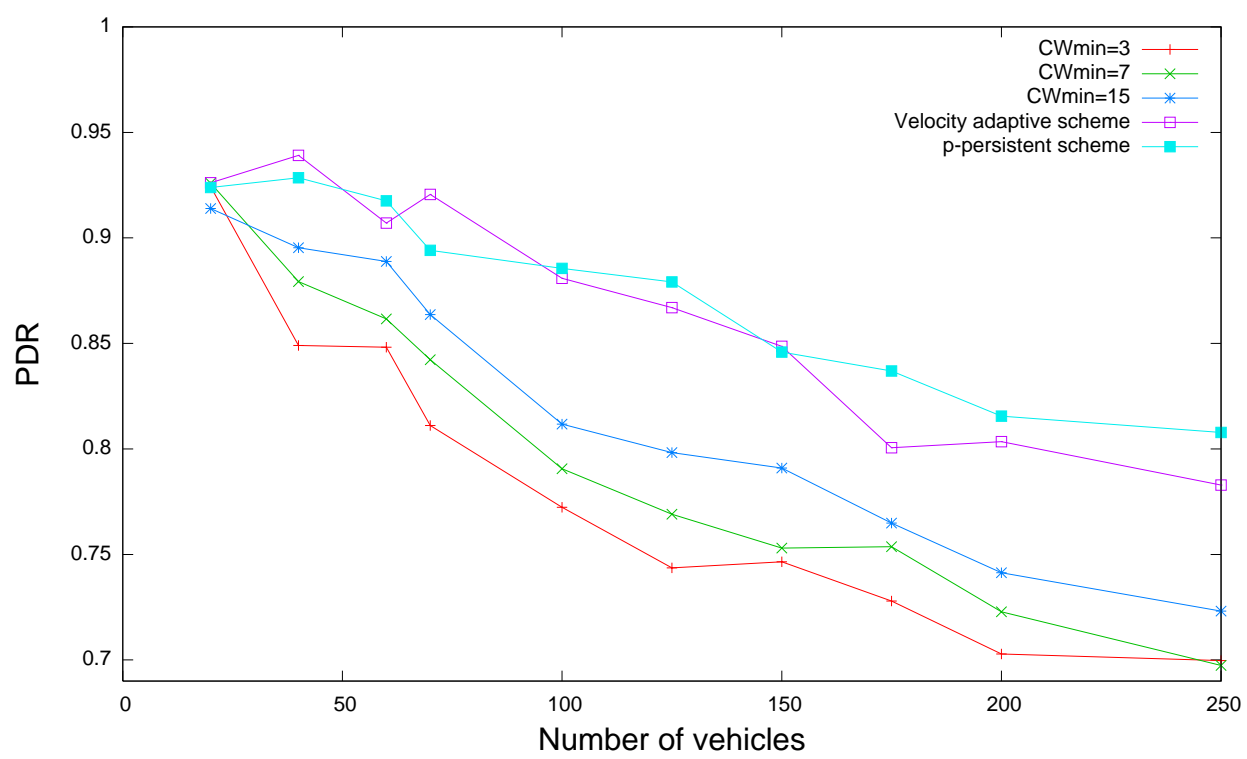

(a) Constant packet arrival rate

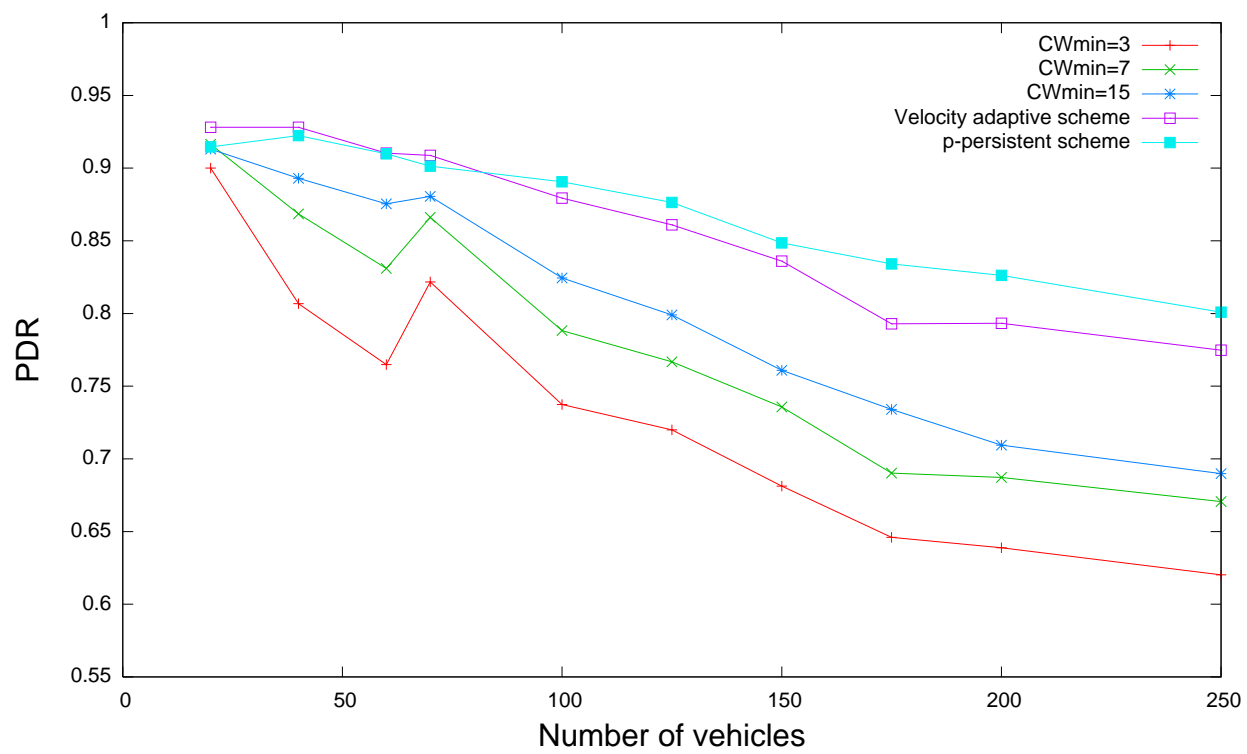

(b) Poisson packet arrival rate

Figure 4.16: PDR of the adaptive MAC schemes.

in Figures 4.17(a) and 4.18(a)

Interestingly, Figure 4.20(a) shows that Jain fairness index indicates high fairness values 


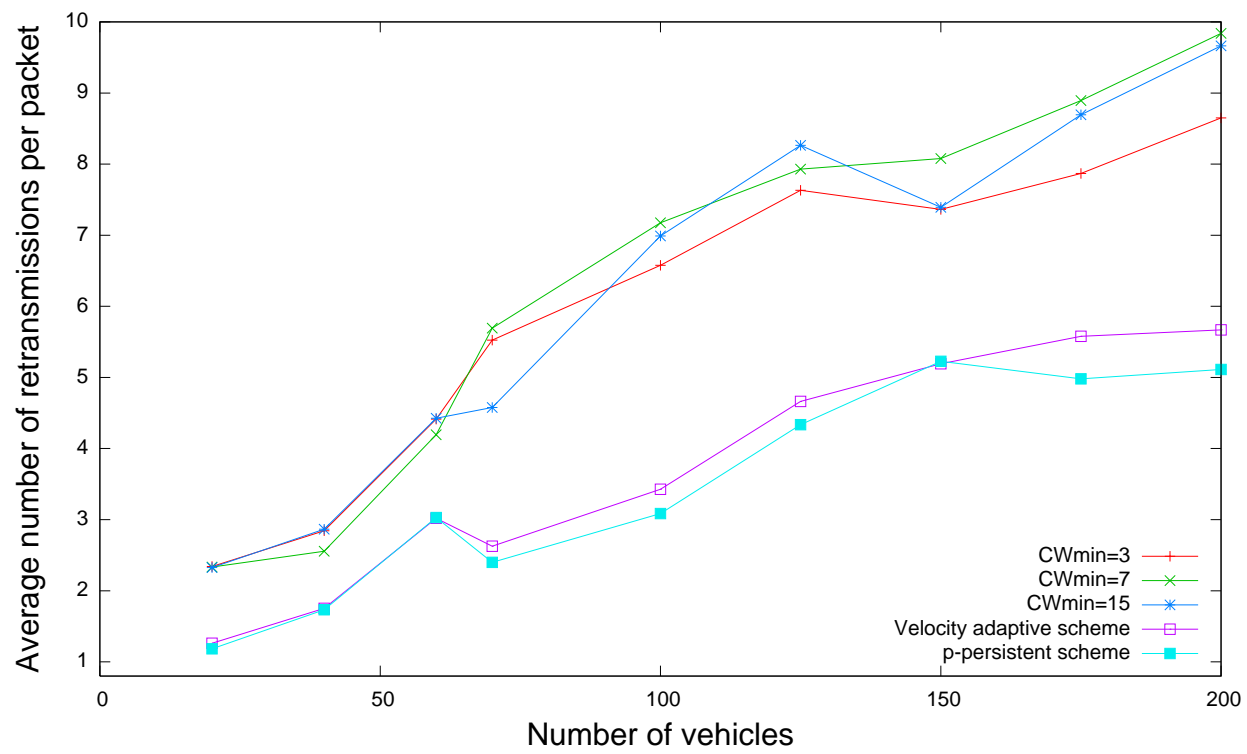

(a) Constant packet arrival rate

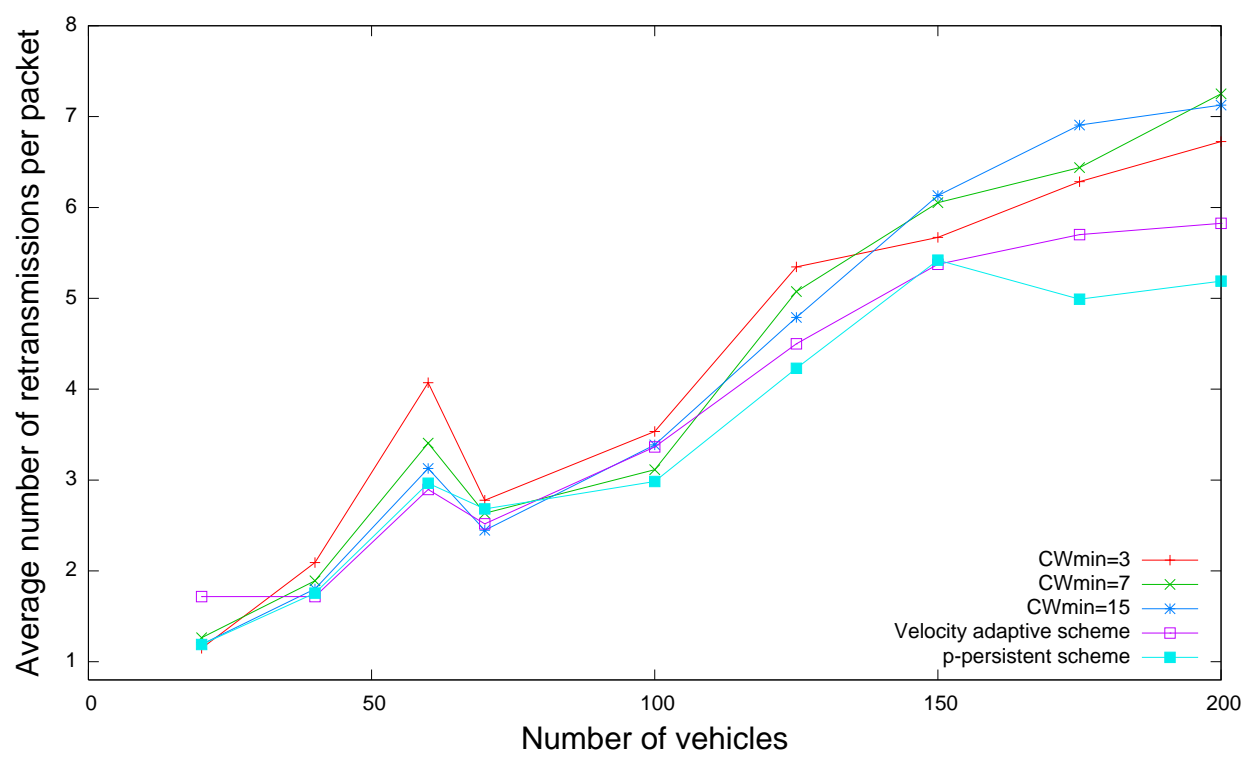

(b) Poisson packet arrival rate

Figure 4.17: Average number of retransmissions of the adaptive MAC schemes.

for the two proposed schemes at node densities less than 150 nodes. For number of nodes 150, 200, and 250, the IEEE $802.11 p$ reveals better fairness than that of the $p$-persistent 


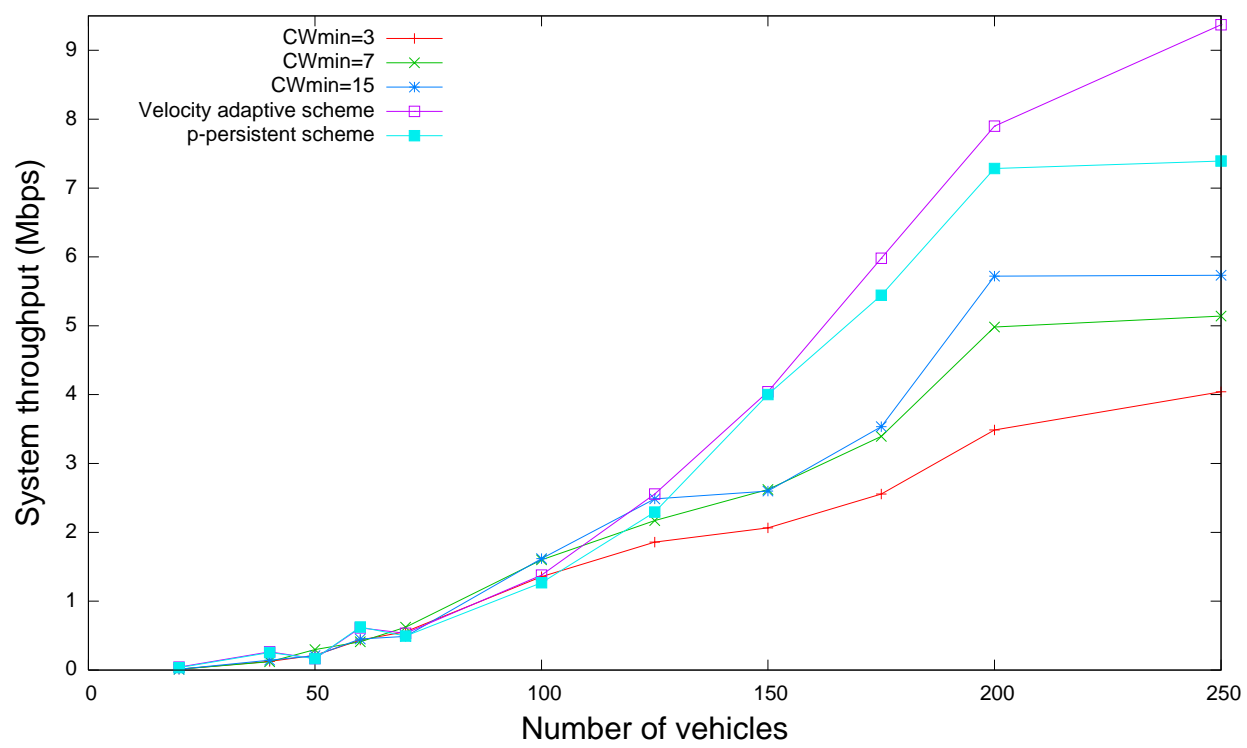

(a) Constant packet arrival rate

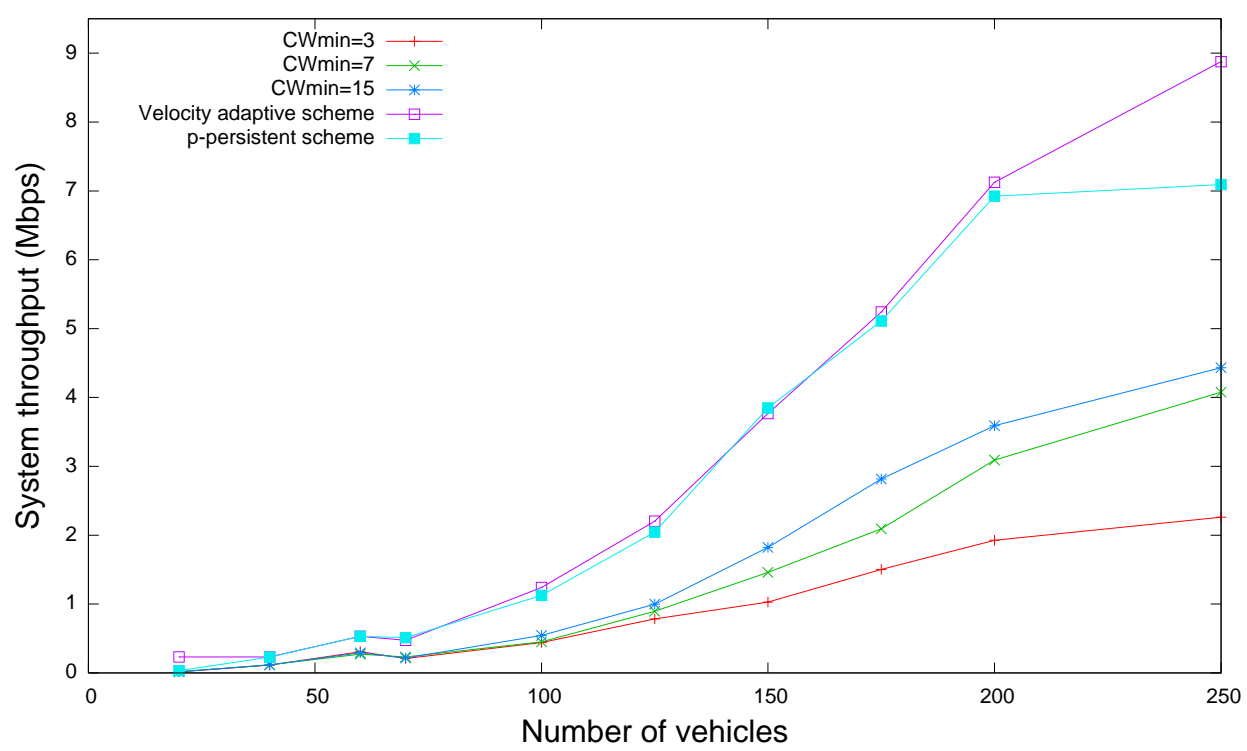

(b) Poisson packet arrival rate

Figure 4.18: Throughput of the adaptive MAC schemes.

and the velocity adaptive schemes. However, the proposed schemes still reveal high fairness of more than $88 \%$. The fact that both proposed schemes result in a similar behavior in 


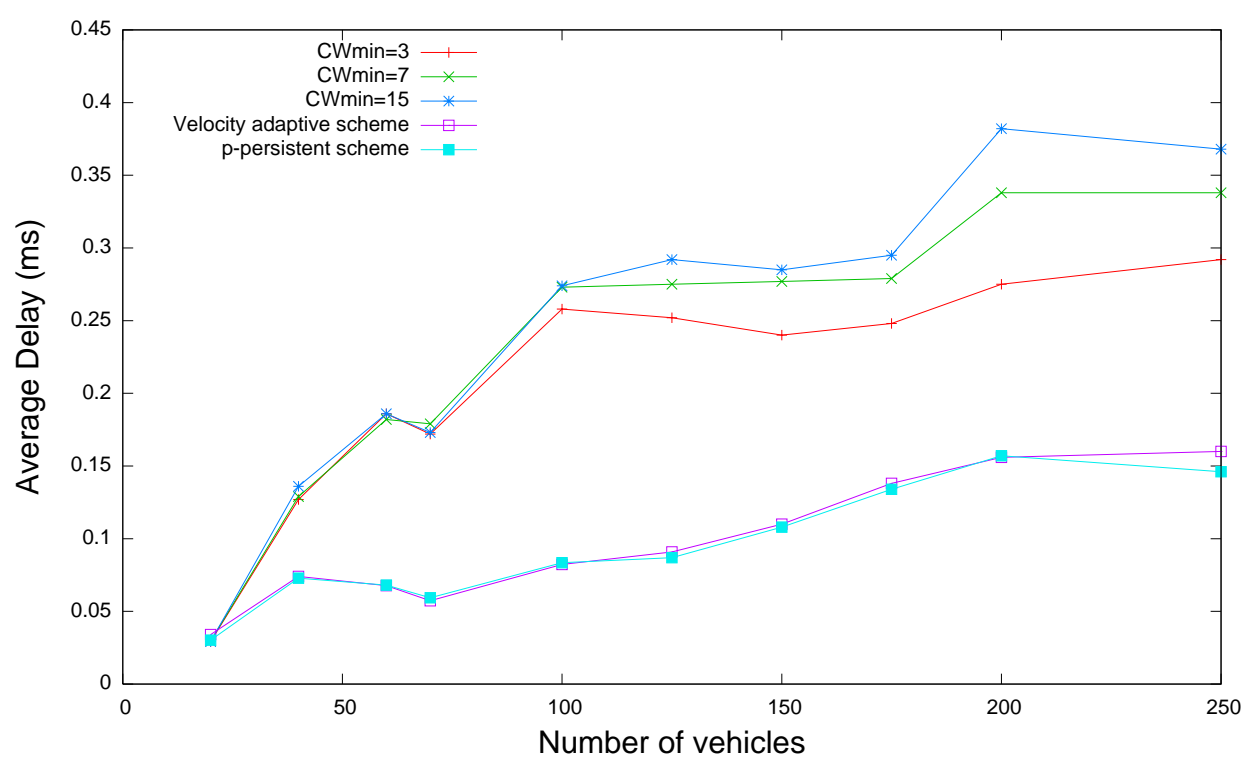

(a) Constant packet arrival rate

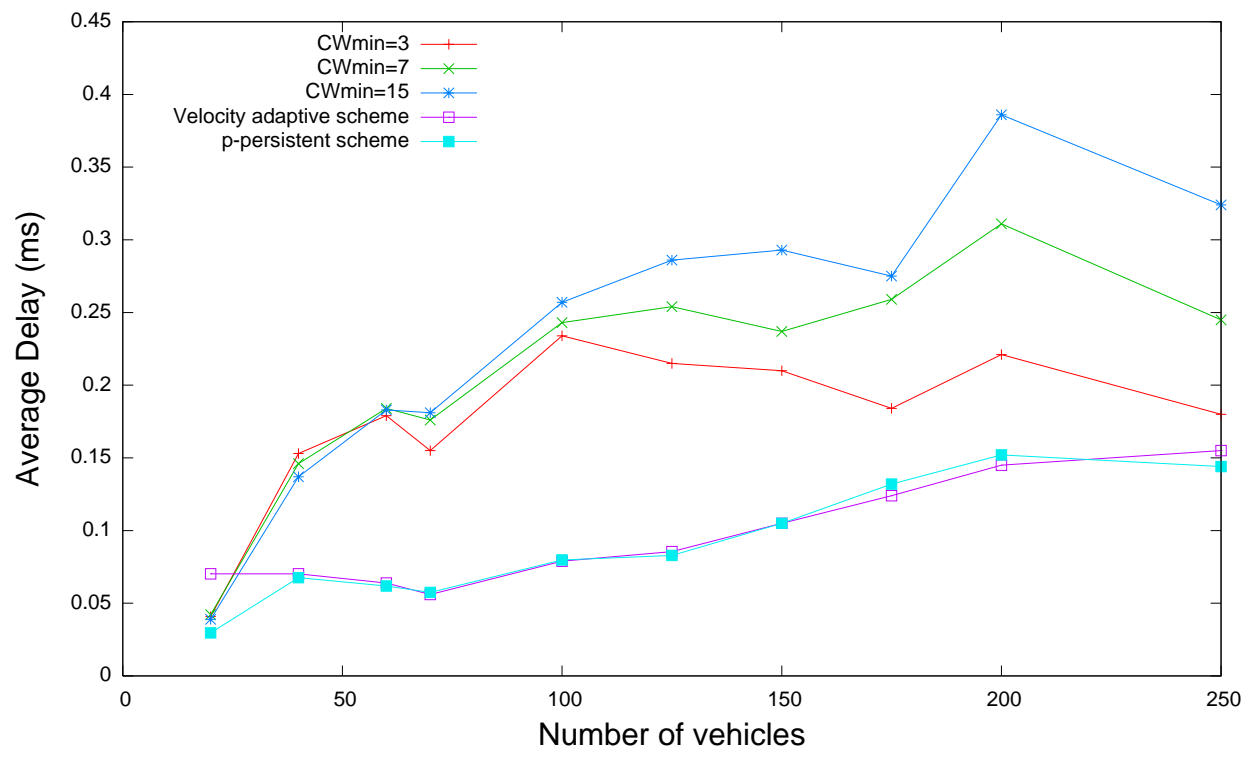

(b) Poisson packet arrival rate

Figure 4.19: Transmission delay of the adaptive MAC schemes.

terms of fairness and delay in almost all node densities, result in different behavior in terms of throughput, and level of contention especially at a high node density is as follows. Both 


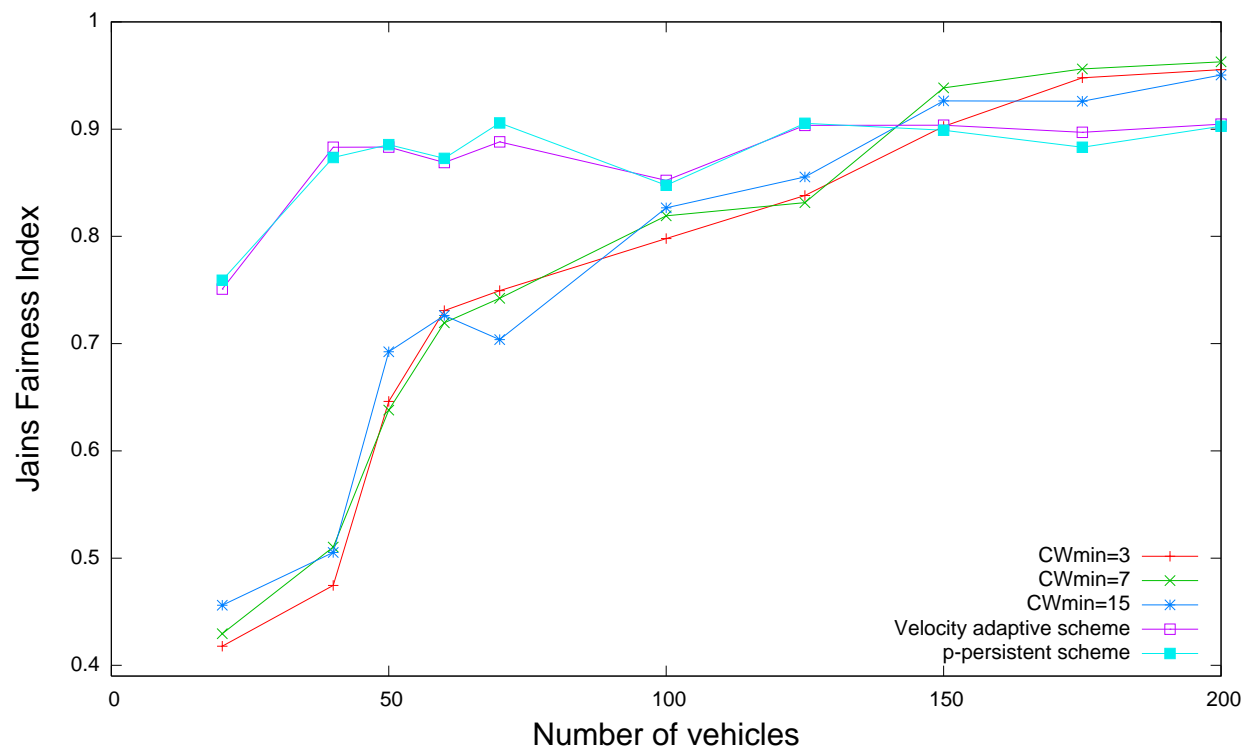

(a) Constant packet arrival rate

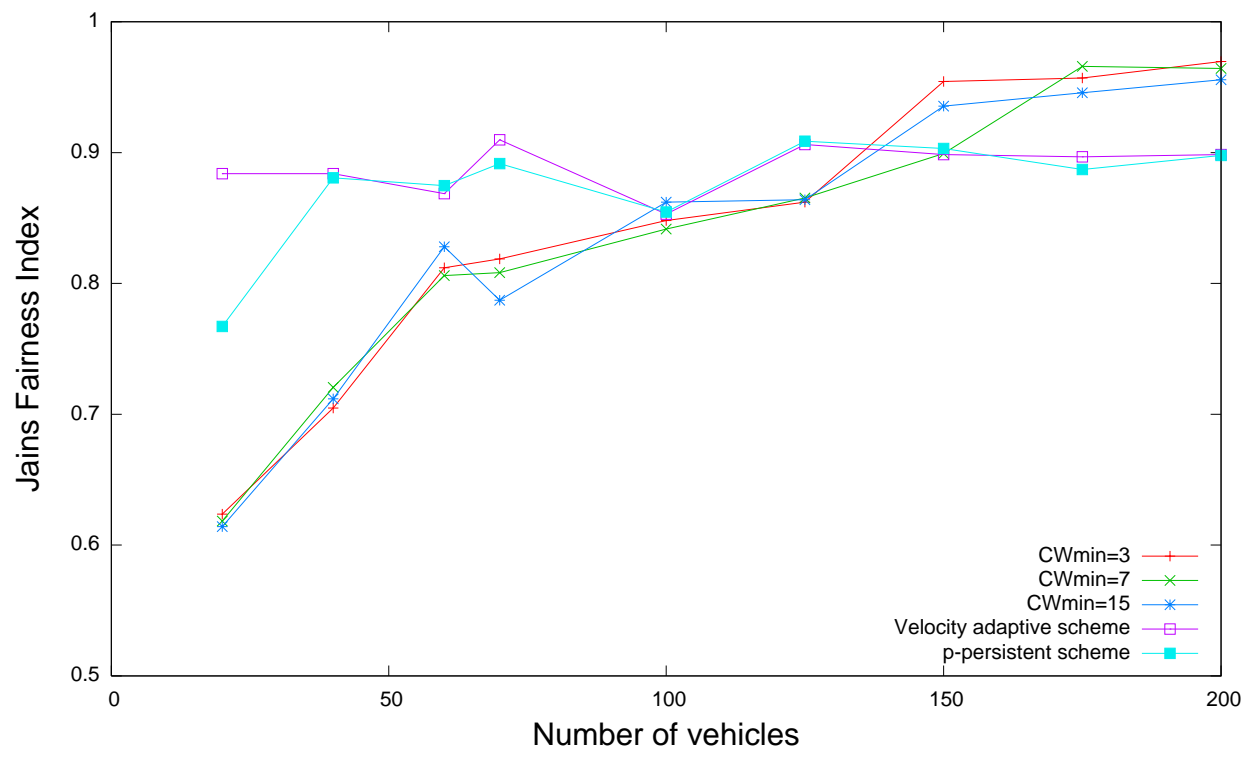

(b) Poisson packet arrival rate

Figure 4.20: Jain Fairness Index of the adaptive MAC schemes.

schemes reduce the level of contention by reducing the number of high priority packets through the dynamic assignment of service priority. By reducing the contention level, 
the number of collisions is reduced, packet dropping rate is decreased, and throughput is increased. Since the $p$-persistent scheme reveals large contention window sizes at a high node density, it provides better management of the contention level. Therefore, it reveals better (or at some node densities, similar) behavior than the velocity adaptive scheme. However, the velocity adaptive scheme outperforms the $p$-persistent scheme in terms of throughput at number of nodes 150, 200, and 250. In sum, reducing the level of contention may result in better performance than that in the IEEE $802.11 \mathrm{p}$, but to achieve the best performance, the MAC protocol should consider the other mobility parameters in VANETs.

For the Poisson packet arrival rate, both proposed schemes outperform the IEEE $802.11 \mathrm{p}$ standard in terms of packet delivery rate as Figure 4.17(b) shows. Moreover, the $p$-persistent scheme results are similar to the velocity adaptive scheme at a low node density and better than the velocity scheme at number of nodes 100 or higher. The average number of retransmissions is slightly improved than that in the IEEE 802.11p as in Figure 4.18(b), Average throughput is significantly improved as Figure 4.19(b) shows. Figure 4.20(b) shows the improvement in the transmission delay. In terms of fairness, the IEEE 802.11p shows a better fairness with Poisson packet arrival rate however, both proposed schemes show high fairness than that of the IEEE 802.11 p at a low node density. At a high node density, the IEEE 802.11p shows slightly better fairness than that of the proposed schemes. However, both schemes provide high fairness index of at least $88 \%$.

\subsection{Summary}

In this chapter, we present the simulation-based evaluation of the IEEE $802.11 \mathrm{p}$ in the broadcast and unicast scenarios. First, we evaluate the impact of mobility factors at the 
MAC level in the broadcast scenario. The results show that relative node speed has a significant impact on the channel access. On the other hand, the distance between the transmitter and receiver does not have such a significant impact. Once a node is in the transmission range of the sender/receiver, it is given a channel time that depends on the other mobility factors. Then, evaluation of the mobility impact at the MAC layer in the unicast scenario shows a severer impact of the relative speed on the medium access. Despite the number of communicating vehicles, most of the vehicles with relative speed less than or equal $1 \mathrm{~m} / \mathrm{s}$ have the largest portion of medium access time. Moreover, an evaluation of the system performance based on throughput, PDR, average number of retransmissions per packet, delay, and fairness index is provided. Finally, simulation results for the adaptive schemes are presented. 


\section{Chapter 5}

\section{Conclusions and Future Work}

The performance of vehicular communication networks depends on several factors. One of the major factors is how the vehicles share the channel time at the MAC level. An efficient and fair medium access in VANETs should result in a reliable packet delivery and a small delay. This can be achieved by considering the mobility model of the vehicles, and taking into account the mobility impact at the MAC level.

In this chapter, we summarize the research contributions and briefly discuss the possible further research work.

\subsection{Research Contributions}

The aim of this thesis is to develop a distributed MAC scheme to provide effective bandwidth sharing for a VANET environment. The main research contributions are as follows:

- We provide a comprehensive evaluation of the mobility impact on the standard IEEE 802.11p performance in the VANET environment. The mobility metrics measure 
the mobility impact on the link connectivity by considering the relative information between vehicles. Relative velocities and positions, the distribution of communication duration, and the distribution of the number of potential neighbors are used as the mobility metrics. Moreover, the performance of the IEEE 802.11p is evaluated according to our performance metrics. The performance metrics include average throughput, PDR, average number of retransmissions, delay, and the fairness index.

- We identified the effective mobility factors that have significant impact on the MAC level. According to our simulation, vehicles relative velocities have a significant impact on the channel access time at the MAC level. In contrast, node relative position does not hve such an impact. However, relative node position can be used to indicate the expected link expiration, which is very frequent in a VANET.

- To the best of our knowledge, we are the first to identify the unfairness problem according to the relative velocities among vehicles in the V2V, infrastructureless, communication mode. Through our extensive simulation, we find out that vehicles with a small relative velocity gain a large part of the medium access, and vice versa. By realizing that, a new unfairness measurement should be addressed in VANET MAC performance.

- Based on the IEEE 802.11 DCF, we propose two dynamic priority management schemes that are adaptive to the significant mobility factors. Both schemes take into consideration the mobility information exchanged periodically among the vehicles. Then, the protocols perform dynamic priority assignment according to the available mobility information. 


\subsection{Future Work}

In our work, we focus on studying the impact of mobility on the IEEE 802.11p MAC. We basically provid a simulation-based evaluation of the impact of the mobility factors on the medium access. Although this thesis provides a comprehensive study and evaluation from different perspectives, there are still some open issues and several research directions that can be pursued to improve the performance of the IEEE 802.11p in VANETs.

Mobility index: In our work, we study the performance of the IEEE 802.11p and evaluate the mobility impact on its performance. The impact is proven to exist. However,

a more precise measure that can indicate the mobility state of the node in the network according to its current state would help to enhance the MAC performance. This measure should provide different communication priority for the vehicles based on their mobility and provide better fairness.

Fuzzy adaptiveness: The dynamic priority management used in our research assumes exact classification of relative speeds into different categories. This assumption is used in the evaluation of the velocity adaptive scheme. However, in reality, it is difficult to determine if the relative speed is very high or very low. There is always a fuzzy region between the different classes of relative speed. Therefore, to avoid crisp assignment and to translate the knowledge from uncertain form to a meaningful output, fuzzy logic based soft computing is expected to give better priority management.

Joint analysis: In our work, we assume a routing protocol that is not based on the mobility factors. A more thorough model should benefits from the same evaluation in a cross-layer design with the upper and/or lower layers. For example, a joint routing and MAC performance analysis based on mobility metrics, such as relative speeds and positions, 
is expected to provide better end-to-end delivery and QoS provisioning. 


\section{Bibliography}

[1] H. Menouar, F. Filali, and M. Lenardi. A survey and qualitative analysis of mac protocols for vehicular ad hoc networks. IEEE Wireless Communications, 13(5):3035, 2006. 1, 8, 10, 26

[2] Q. Xu, T. Mak, J. Ko, and R. Sengupta. Vehicle-to-vehicle safety messaging in DSRC. In Proceedings of the 1st ACM international workshop on Vehicular ad hoc networks, pages 19-28, 2004. 2, 10, 28

[3] E. Karamad and F. Ashtiani. A modified 802.11-based MAC scheme to assure fair access for vehicle-to-roadside communications. Computer Communications, 31(12):2898-2906, 2008. 3, 4, 21, 36

[4] ASTM E 2213-03. Standard Specification for Telecommunications and Information Exchange between Roadside and Vehicle Systems-5.9 GHz Band Dedicated Short Range Communications (DSRC) Medium Access Control (MAC) and Physical Layer (PHY) Specifications, ASTM International. 4, 17, 18, 36

[5] X. Chen, H.H. Refai, and X. Ma. On the enhancements to IEEE 802.11 MAC and their suitability for safety-critical applications in VANET. Wireless Communications and Mobile Computing, 2008. 4, 18, 19, 26, 35 
[6] S. Eichler. Performance Evaluation of the IEEE 802.11p WAVE Communication Standard. IEEE Vehicular Technology Conference, pages 2199-2203, 2007. 4, 18, 20, 35. 36. 65

[7] Y. Wang, A. Ahmed, B. Krishnamachari, and K. Psounis. IEEE 802.11p performance evaluation and protocol enhancement. IEEE International Conference on Vehicular Electronics and Safety, pages 317-322, 2008. 4, 19, 35, 36

[8] T. Murray, M. Cojocari, and H. Fu. Measuring the performance of IEEE 802.11p using ns-2 simulator for vehicular networks. IEEE International Conference on Electro/Information Technology, pages 498-503, 2008. 4, 18, 21

[9] H. Hartenstein and K.P. Laberteaux. A tutorial survey on vehicular ad hoc networks. IEEE Communications Magazine, 46(6):164-171, 2008. 8, 17

[10] F. Borgonovo, A. Capone, M. Cesana, and L. Fratta. ADHOC: a new, flexible and reliable MAC architecture for ad-hoc networks. IEEE Wireless Communications and Networking, pages 965-970. v. 2, 2003. 8, 12,26

[11] X. Long and B. Sikdar. MAC and Routing Protocols for Vehicle to Vehicle Communications. Automotive Informatics and Communicative Systems: Principles in Vehicular Networks and Data Exchange, 2009. 12

[12] T. Kosch, C.J. Adler, S. Eichler, C. Schroth, and M. Strassberger. The scalability problem of vehicular ad hoc networks and how to solve it. IEEE Wireless Communications, 13(5):22-28, 2006. 12

[13] M.S. Gast. 802.11 Wireless Networks: The Definitive Guide, 2005. 14 
[14] Standards Committee. Wireless LAN Medium Access Control (MAC) and Physical layer (PHY) specifications: Amedment 8: Medium Access Control (MAC) Quality of Service Enhancments, IEEE Computer Socitey, 2005. 15

[15] http://www.standards.its.dot.gov/, 17

[16] L. Stibor, Y. Zang, and H.J. Reumerman. Neighborhood evaluation of vehicular adhoc network using IEEE 802.11 p. European Wireless Conference, 2007. 18, 20, 36, 38

[17] C. Cuyu, Y. Xiang, S. Meilin, and L. Lin. Performance Observations on MAC Protocols of VANETs in Intelligent Transportation System. International Conference on Communications and Mobile Computing, pages 373-379. v. 2, 2009. 19, 26, 35

[18] K. Bilstrup, E. Uhlemann, E.G. Strom, and U. Bilstrup. Evaluation of the IEEE 802.11p MAC Method for Vehicle-to-Vehicle Communication. IEEE Vehicular Technology Conference, pages 1-5, 2008. 19, 35

[19] D.D. Perkins, H.D. Hughes, and C.B. Owen. Factors affecting the performance of ad hoc networks. IEEE International Conference on Communications, pages 2048-2052. v. 4, 2002. 20

[20] N. Choi, S. Choi, Y. Seok, T. Kwon, and Y. Choi. A Solicitation-based IEEE 802.11p MAC Protocol for Roadside to Vehicular Networks. Mobile Networking for Vehicular Environments, pages 91-96, 2007. 20, 21

[21] B.S. Gukhool and S. Cherkaoui. Handoff in IEEE 802.11p-based vehicular networks. International Conference on Wireless and Optical Communications Networks, pages $1-5,2009.21$ 
[22] Y. Gunter, B. Wiegel, and H.P. Grossmann. Medium Access Concept for VANETs Based on Clustering. IEEE Vehicular Technology Conference, pages 2189-2193, 2007. 22. 23

[23] J.Y. Yu and P.H.J. Chong. A survey of clustering schemes for mobile ad hoc networks. IEEE Communications Surveys \& Tutorials, 7(1):32-48, 2005. 22

[24] H. Su and X. Zhang. Clustering-Based Multichannel MAC Protocols for QoS Provisionings Over Vehicular Ad Hoc Networks. IEEE Transactions on Vehicular Technology, 56(6):3309-3323, 2007. 23, 24

[25] Z.Y. Rawashdeh and S.M. Mahmud. Media Access Technique for Cluster-Based Vehicular Ad Hoc Networks. IEEE Vehicular Technology Conference, pages 1-5, 2008. 24

[26] S.V. Bana and P. Varaiya. Space division multiple access (SDMA) for robust ad hoc vehicle communication networks. IEEE Intelligent Transportation Systems, pages 962-967, 2001. 24

[27] S. Katragadda, G. Murthy, R. Rao, M. Kumar, and R. Sachin. A decentralized location-based channel access protocol for inter-vehicle communication. IEEE Semiannual Vehicular Technology Conference, pages 1831-1835 v. 3, 2003. 24

[28] J.J. Blum and A. Eskandarian. A Reliable Link-Layer Protocol for Robust and Scalable Intervehicle Communications. IEEE Transactions on Intelligent Transportation Systems, 8(1):4-13, 2007. 24

[29] X. Chen and H.H. Refai. SDMA: On The Suitability for VANET. International 
Conference on Information and Communication Technologies: From Theory to Applications, pages 1-5, 2008. 25

[30] D. Lee, R. Attias, A. Puri, R. Sengupta, S. Tripakis, and P. Varaiya. A wireless token ring protocol for intelligent transportation systems. IEEE Intelligent Transportation Systems, pages 1152-1157, 2001. 25

[31] M. Ergen, D. Lee, R. Sengupta, and P. Varaiya. WTRP - wireless token ring protocol. IEEE Transactions on Vehicular Technology, 53(6):1863-1881, 2004. 25

[32] J. Zhang, K.-H. Liu, and X. Shen. A Novel Overlay Token Ring Protocol for InterVehicle Communication. IEEE International Conference on Communications, pages 4904-4909, 2008. 25

[33] H. Dai, K.-W. Ng, and M.-Y. Wu. An Overview of MAC Protocols with Directional Antennas in Wireless ad hoc Networks. International Conference on Wireless and Mobile Communications, pages 84-84, 2006. 27

[34] T. Korakis, G. Jakllari, and L. Tassiulas. CDR-MAC: A Protocol for Full Exploitation of Directional Antennas in Ad Hoc Wireless Networks. IEEE Transactions on Mobile Computing, 7(2):145-155, 2008. 27

[35] R.M. Yadumurthy, A.C. H., M. Sadashivaiah, and R. Makanaboyina. Reliable MAC broadcast protocol in directional and omni-directional transmissions for vehicular ad hoc networks. In Proceedings of the 2nd ACM international workshop on Vehicular ad hoc networks, pages 10-19, 2005. 28

[36] X. Xie, F. Wang, H. Wang, and K. Li. Adaptive Multi-channel MAC Protocol for 
Dense VANET Using Directional Antennas. Second International Conference on Future Generation Communication and Networking, pages 398-401. v. 2, 2008. 28

[37] K.-T. Feng. LMA: Location- and Mobility-Aware Medium-Access Control Protocols for Vehicular Ad Hoc Networks Using Directional Antennas. IEEE Transactions on Vehicular Technology, 56(6):3324-3336, 2007. 28

[38] M. Sadashivaiah, R. Makanaboyina, B. George, and R. Raghavendra. Performance evaluation of directional MAC protocol for inter-vehicle communication. IEEE Vehicular Technology Conference, pages 2585-2589 v. 4, 2005. 28

[39] Q. Xu, T. Mak, J. Ko, and R. Sengupta. Medium Access Control Protocol Design for Vehicle Vehicle Safety Messages. IEEE Transactions on Vehicular Technology, $56(2): 499-518,2007.28$

[40] F. Farnoud, B. Hassanabadi, and S. Valaee. Message Broadcast using Optical Orthogonal Codes in Vehicular Communication Systems. Wireless Networking for Intelligent Transportation Systems Workshop, 2007. 29

[41] F. Farnoud and S. Valaee. Repetition-based broadcast in vehicular ad hoc networks in Rician channel with capture. IEEE INFOCOM Workshops, pages 1-6, 2008. 29

[42] B. Hassanabadi, L. Zhang, and S. Valaee. Index Coded Repetition-Based MAC in Vehicular Ad-Hoc Networks. IEEE Consumer Communications and Networking Conference, pages 1-6, 2009. 29

[43] Z. Wang and M. Hassan. How much of dsrc is available for non-safety use? In Proceedings of the fifth ACM international workshop on VehiculAr Inter-NETworking, pages 23-29, 2008. 30 
[44] T.K. Mak, K.P. Laberteaux, and R. Sengupta. A multi-channel VANET providing concurrent safety and commercial services. In Proceedings of the 2nd ACM international workshop on Vehicular ad hoc networks, pages 1-9, 2005. 30

[45] T.K. Mak, K.P. Laberteaux, R. Sengupta, and M. Ergen. Multichannel Medium Access Control for Dedicated Short-Range Communications. IEEE Transactions on Vehicular Technology, 58(1):349-366, 2009. 30

[46] S.-E. Chung, J. Yoo, and C.-K. Kim. A cognitive MAC for VANET based on the WAVE systems. International Conference on Advanced Communication Technology, pages 41-46. v. 1, 2009. 31

[47] G. Bianchi. Performance analysis of the IEEE 802. 11 distributed coordination function. IEEE Journal on selected areas in communications, 18(3):535-547, 2000. 40

[48] D.J. Deng, C.H. Ke, H.H. Chen, and Y.M. Huang. Contention window optimization for IEEE 802.11 DCF access control. IEEE Transactions on Wireless Communications, 7(12):5129-5135, 2008. 40

[49] J. Yin, X. Wang, and D.P. Agrawal. Optimal packet size in error-prone channel for IEEE 802.11 distributed coordination function. In Proceedings of IEEE WCNC, volume 3, pages 1654-1659, 2004. 40

[50] B. Xiang and M. Yu-Ming. A simple adaptive p-persistent MAC scheme for service differentiation and maximum channel utilization. WSEAS Transactions on Communications, 7:1-7, 2007. 40

[51] F. Calì, M. Conti, and E. Gregori. Dynamic tuning of the IEEE 802.11 protocol 
to achieve a theoretical throughput limit. IEEE/ACM Transactions on Networking (TON), 8(6):799, 2000. 40, 41, 42

[52] K. Yedavalli and B. Krishnamachari. Enhancement of the IEEE 802.15. 4 MAC protocol for scalable data collection in dense sensor networks. In Modeling and Optimization in Mobile, Ad Hoc, and Wireless Networks and Workshops, pages 152-161, 2008. 40. 42

[53] F. Bai, N. Sadagopan, and A. Helmy. Important: A framework to systematically analyze the impact of mobility on performance of routing protocols for adhoc networks. IEEE INFOCOM, 2003. 46, 47

[54] K. Fall and K. Varadhan. The ns Manual (formerly ns Notes and Documentation), The VINT Project. 47

[55] D. Chiu R. Jain, W. Hawe. A Quantitative measure of fairness and discrimination for resource allocation in Shared Computer Systems. 1984. 51 